TRANSACTIONS OF THE

AMERICAN MATHEMATICAL SOCIETY

Volume 353, Number 9, Pages 3409-3464

S 0002-9947(01)02774-X

Article electronically published on May 4, 2001

\title{
FINITE STATE AUTOMATA: A GEOMETRIC APPROACH
}

\author{
BENJAMIN STEINBERG
}

\begin{abstract}
Recently, finite state automata, via the advent of hyperbolic and automatic groups, have become a powerful tool in geometric group theory. This paper develops a geometric approach to automata theory, analogous to various techniques used in combinatorial group theory, to solve various problems on the overlap between group theory and monoid theory. For instance, we give a geometric algorithm for computing the closure of a rational language in the profinite topology of a free group. We introduce some geometric notions for automata and show that certain important classes of monoids can be described in terms of the geometry of their Cayley graphs. A long standing open question, to which the answer was only known in the simplest of cases (and even then was non-trivial), is whether it is true, for a pseudovariety of groups $\mathbf{H}$, that a $\mathcal{J}$-trivial co-extension of a group in $\mathbf{H}$ must divide a semidirect product of a $\mathcal{J}$-trivial monoid and a group in $\mathbf{H}$. We show the answer is affirmative if $\mathbf{H}$ is closed under extension, and may be negative otherwise.
\end{abstract}

\section{INTRODUCTION}

The geometry of the Cayley graph of a group is key to most modern approaches to group theory. An automaton, on the surface, is much like a Cayley graph and should be amenable to similar techniques. This paper explores some applications of techniques from geometric group theory to the theory of finite automata and monoids. The approach of this paper is to first rigorously develop the fundamentals of the theory, and then to apply the theory to various problems. We attempt to keep the exposition fairly self-contained, reproving some old results from a new, geometric point of view.

While a connection between group theory and monoid theory has always existed, the interplay between these two subjects has only truly become apparent in this decade. On the one hand, the theory of automatic groups [15] is an attempt to use finite monoids to understand the structure of a group; on the other hand, in recent years, several deep results in semigroup theory have come from (and motivated) group-theoretic results. The best example of this is the theorem of Ribes and Zalesskiı 37. that products of finitely generated subgroups of a free group are closed in the profinite topology. This theorem was conjectured by semigroup theorists [34] because its truth implied the truth of the Rhodes Type II Conjecture which had already been verified in several important cases. This conjecture was then used to

Received by the editors February 12, 1999 and, in revised form, August 24, 2000.

1991 Mathematics Subject Classification. Primary 20M35, 20F10.

Key words and phrases. Immersions, coverings, fundamental groups, profinite topologies, rational languages, automata, graphs, monoids, block groups, semidirect products, pseudovarieties, $\mathrm{Mal}^{\prime} \mathrm{cev}$ products.

The author was supported in part by Praxis XXI scholarship BPD 1630698. 
prove, amongst other things, one of the most difficult results in finite semigroup theory to date: the seemingly innocuous equation $\mathbf{P G}=\mathbf{B G}$ which states that every block group divides a power group. The techniques used by Ribes and Zalesskiǔ involved profinite groups acting on profinite trees, techniques seemingly far removed from the primary application: the calculation of the closure of a rational (or regular) language in the profinite topology, something very important in monoid theory.

Another such example is the interpretation of Stallings' [40], including the proof of Marshall Hall's Theorem, as statements about inverse automata by Margolis and Meakin [28. In particular, they classify all immersions of a finite graph in a manner similar to the classification of covering spaces, but using free inverse monoids rather than free groups. Also one can show, see 29 for a survey, that a finitely generated subgroup of a free group is pure if and only if its associated immersion, viewed as an inverse automaton, is aperiodic; hence this is a decidable property. This interplay between group theory and inverse automata theory is further explored by Margolis, Sapir, and Weil in 30] to relate an embedding problem in the theory of immersions to a membership problem for pseudovarieties of inverse monoids.

This paper applies the techniques of graph immersions and coverings to monoids and automata in general-the results are rather surprising. First of all, we obtain an elementary and more general version of the results of [30. In addition, using the Ribes and Zalesskiu Theorem [37, we obtain a simple geometric procedure to compute the closure of a rational language in the profinite topology on a free group. In particular, we discover that some special cases of the Type II Theorem, for example the case of regular monoids, follow from Hall's Theorem which is much more elementary than the theorem of Ribes and Zalesski1. Our algorithm is internal to the automaton accepting the language and has the benefit that, from the automaton, we construct a non-deterministic automaton which can be used to recognize the closure of every language recognized by the original automaton. In applications, this is the situation in which one finds oneself, and so we obtain a true increase in efficiency.

While clarification of old results is important, a new theory should also lead to new results-and so our theory does. Our main result is related to the problem of extensions in group theory. Recall that $G$ is an extension of $N$ by $H$ if there is a surjective homomorphism $\varphi: G \rightarrow H$ with $1 \varphi^{-1}=N$ (one also says that $G$ is a co-extension of $H$ by $N$ ). The extension is said to be split if $G=N * H$ where $*$ denotes the semidirect product (rather than the free product). The relation between extensions and split extensions is that if $G$ is any extension of $N$ by $H$, then $G$ can be embedded in $N \circ H$ where $\circ$ denotes the wreath product, which is nothing more than a semidirect product of the form $N^{H} * H$. More generally, if $\varphi: M \rightarrow G$ is a surjective morphism with $M$ a monoid and $G$ a group, then $N=1 \varphi^{-1}$ is a submonoid of $M$. In this case, we say that $M$ is a co-extension of $G$ by $N$. One can also define the semidirect product $N * G$ of a monoid $N$ and a group $G$ and, in this case, $N * G$ is a co-extension of $G$ by $N$. Unfortunately, not every co-extension of a group can be recovered by a wreath product. It was the study of co-extensions that motivated the conjecture of [34] which led to the Ribes and Zalesskii Theorem.

To discuss this problem further, we need the notion of a pseudovariety of monoids, introduced in the seventies by Eilenberg [14] as a means by which to classify finite monoids. A pseudovariety of monoids is a class of finite monoids closed under 
forming finite products, taking submonoids, and taking quotient monoids. Since there are far too many finite monoids to classify them up to isomorphism (think of how many multiplication tables one can put on an 8 element set, including 0 , satisfying the identity $x y z=0$ ), Eilenberg [14], motivated by the Krohn-Rhodes Theorem [17] and the Theory of Complexity [18, pushed for the classification of finite monoids into pseudovarieties. From this viewpoint, it is then natural to define, for $\mathbf{V}$ a pseudovariety of monoids and $\mathbf{H}$ a pseudovariety of groups, the pseudovarieties of monoids $\mathbf{V} * \mathbf{H}$ (the semidirect product) and $\mathbf{V} \rightarrow \mathbf{H}$ (the Mal'cev product) generated, respectively, by semidirect products of monoids in $\mathbf{V}$ with groups in $\mathbf{H}$ and by co-extensions of groups in $\mathbf{H}$ by monoids in $\mathbf{V}$. In general, $\mathbf{V} * \mathbf{H} \subseteq \mathbf{V} m \mathbf{H}$, while the reverse inclusion follows if $\mathbf{V}$ is also a pseudovariety of groups by the observation above concerning wreath products. There are many pseudovarieties of monoids for which equality holds, for instance, if $\mathbf{V}=\mathbf{S l}$, the pseudovariety of semilattices (idempotent-commutative monoids); there is even a well-known sufficient condition for equality. Since semidirect products can be understood in terms of their factors, this is the desired situation. However, there is an important example, that of $\mathcal{J}$-trivial monoids, for which this sufficient condition fails. A finite monoid is called $\mathcal{J}$-trivial if each principal two-sided ideal has a unique generator. It turns out, that the $\mathcal{J}$-trivial co-extensions of groups are precisely those finite monoids, called block groups, such that each regular element has a unique inverse. A deep result of Henckell and Rhodes [24] says that $\mathbf{J} * \mathbf{G}=\mathbf{J}: \mathbf{G}$ where $\mathbf{J}$ is the pseudovariety of all finite $\mathcal{J}$-trivial monoids and $\mathbf{G}$ is the pseudovariety of all finite groups. However, one also wants to study, for instance, $\mathcal{J}$-trivial co-extensions of a p-group, and so it is important to know whether such a monoid can "be obtained" from a semidirect product. Let $\mathbf{H}$ be a pseudovariety of groups closed under extensions, or, equivalently, forming semidirect products; we show in this paper that $\mathbf{J} * \mathbf{H}=\mathbf{J}(\mathrm{m} \mathbf{H}$. The proof is independent of the result for $\mathbf{G}$ and when restricted to that case gives the shortest, least technical proof to date. The result uses a generalization by Ribes and Zalesskii [38] of their theorem to the pro-H topology, and that the geometry of the Cayley graph of a block group is particularly well suited to our methods. Since the author has already shown that $\mathbf{J} * \mathbf{H}$, in the extensionclosed setting, is generated by power sets of groups in $\mathbf{H}$, viewed as monoids under setwise products [45], this result strongly relates the structure of $\mathbf{J} m \mathbf{H}$ to the structure of $\mathbf{H}$. We also show that this equation can indeed fail for pseudovarieties of groups which are not extension-closed. In addition, we show that the $\mathbf{H}$-closed rational languages are recognized by monoids in $\mathbf{J}(m) \mathbf{H}$. Many generalizations to other Mal'cev and semidirect products are obtained as well.

The paper is organized in the following manner. We begin by defining graphs and labeled graphs and studying their geometry; some basic results from combinatorial group theory are surveyed; we then define deterministic and non-deterministic automata geometrically. After a brief digression on monoid theory, we focus on inverse monoid theory; here, we obtain simple geometric proofs of the results of [30] and lay the groundwork for the more general monoid situation. Then follows a study of the geometry of strongly connected automata; we describe the profinite closures of the languages they recognize in terms of the fundamental groups of geometrically related automata. From there, we move to more general automata by studying them in terms of their strongly connected components; we obtain, in particular, our geometric algorithm for computing the profinite closure of a rational language 
as well as a geometric condition on when the profinite and pro-H closures of a language coincide for an extension-closed pseudovariety of groups $\mathbf{H}$. Our results are then applied to the Cayley graph of a monoid where we demonstrate that certain pseudovarieties of monoids are entirely determined by geometric invariants of the Cayley graphs of their members. The remaining sections apply these results to prove various equalities of pseudovarieties of monoids and to give counterexamples to possible equalities.

\section{Some Conventions and Notation}

Since first and foremost this is a paper concerned with semigroups, monoids, and automata, we are led in general to write our morphisms between algebraic objects on the right. Most other times, arguments will appear in their more customary positions. We shall follow the set theoretic convention of not distinguishing between a relation $\varphi$ of sets $S$ and $T$ viewed as a function $\varphi: S \rightarrow 2^{T}$ and as a set of pairs in $S \times T$. Hence, at various times, one might see such notation as $t \in s \varphi, s \in t \varphi^{-1}$, or $s \varphi t$, all of which mean that $s$ and $t$ are $\varphi$-related. We shall abuse notation when considering morphisms of graphs and categories by using the same symbol to denote the vertex and edge functions. Categories will be viewed as graphs with an algebraic structure and so we shall use words such as vertices and edges instead of objects and arrows. However, it will be convenient to talk of hom sets.

If $A$ is a set, we let $\tilde{A}=A \cup A^{-1}$ where $A$ and $A^{-1}$ are disjoint sets in bijection via a map $a \mapsto a^{-1}$ with the property that $\left(a^{-1}\right)^{-1}=a$ for any $a \in \tilde{A}$. We use $A^{*}$ for the free monoid and $F G(A)$ for the free group generated by a set $A$. For a word

$w \in \tilde{A}^{*}$, we define $w^{-1}$ to be the word obtained in the standard manner by reversing the order of appearance of the letters and replacing each letter by its inverse. In this way, $\tilde{A}^{*}$ becomes the free monoid with involution. We shall not distinguish between a reduced word and the element of the free group which it represents. If $M$ is an $A$-generated algebra of some type, we shall use $[w]_{M}$ to denote evaluation in $M$ of a term $w$ of an appropriate free algebra generated by $A$. Primarily, we shall be interested in monoids, not semigroups. Thus, we shall formulate most of our definitions for monoids, leaving the analogous semigroup definitions to the reader. For undefined concepts from semigroup theory, we refer the reader to [2, 12, 14, and from category theory to [25]. As a final note, many of the results of this paper can be proved making use of profinite monoids. The author used this approach in 44 to prove certain cases of some of the results of this paper. Also many of the deep results which we quote in this paper are proved using profinite groups and profinite graphs. We have avoided using profinite objects deliberately in this paper to make it more accessible. However, one can apply many of the ideas of this paper to profinite groups, monoids, and graphs; for instance, a labeling of a profinite graph by a profinite set can be defined in a fashion analogous to that used for discrete objects in this paper. We leave these generalizations to the interested reader and, indeed, there is already a considerable literature on profinite groups acting on profinite graphs; see [7, 21].

\section{Graphs and Automata}

In this section we introduce our geometric conception of automata, viewing them in a combinatorial or topological fashion. 
3.1. Graphs. By a graph, we mean a graph in the sense of Serre and Stallings [39] 40]. Formally, a graph $\Gamma$ consists of the following data: a set $V(\Gamma)$ of vertices; a set $E(\Gamma)$ of edges; a function $\alpha: E(\Gamma) \rightarrow V(\Gamma)$; and a map on edges $e \mapsto \bar{e}$ such that $\overline{\bar{e}}=e$ and $\bar{e} \neq e$. We define a function $\omega: E(\Gamma) \rightarrow V(\Gamma)$ by $e \omega=\bar{e} \alpha$. One calls, respectively, $e \alpha, e \omega, \bar{e}$ the, respectively, initial vertex, terminal vertex, reverse edge of $e$. Note that $\mathbb{Z} / 2 \mathbb{Z}$ acts freely on $E$ via the involution $e \mapsto \bar{e}$. An orientation of $\Gamma$ consists of a choice of a representative from each $\mathbb{Z} / 2 \mathbb{Z}$-orbit. The representatives are called positively oriented edges. We call a graph $\Gamma$ with a given orientation an oriented or directed graph. If $\Gamma$ is an oriented graph, we use $E^{+}(\Gamma)$ to denote the set of positively oriented edges; then $E(\Gamma)=E^{+}(\Gamma) \cup \overline{E^{+}(\Gamma)}$ and the union is disjoint. An oriented graph is completely determined by its sets of positively oriented edges, by $\alpha$, and by $\omega$. Normally, when one draws an oriented graph, one draws only the positively oriented edges with an arrow from the initial vertex to the terminal vertex. One then thinks of the reverse edge as being the same edge, but traversed in the reverse direction. A graph $\Gamma$ is called finite if $V(\Gamma)$ and $E(\Gamma)$ are finite.

A path $p$ of length $n$ in a graph $\Gamma$ is a sequence of edges $e_{1} \cdots e_{n}$ of $\Gamma$ such that $e_{j} \omega=e_{j+1} \alpha$. The notation $|p|$ will be used to denote the length of $p$. We let $p \alpha=e_{1} \alpha$ and $p \omega=e_{n} \omega$. One then says that $p$ is a path from $p \alpha$ to $p \omega$. If $p \alpha=p \omega$, we call $p$ a circuit or a loop. We also allow an empty loop (of length 0 ) at each vertex $v$, denoted $1_{v}$. If $\Gamma$ is oriented and the $e_{j}$ are all positively oriented, $p$ is called a directed path. By convention, the empty loop is considered to be directed. If $p$ is a path, the reverse path of $p$ is $\bar{p}=\bar{e}_{n} \cdots \bar{e}_{1}$. Note $\bar{p} \alpha=p \omega, \bar{p} \omega=p \alpha$, and $\overline{\bar{p}}=p$. One takes, by convention, $\overline{1_{v}}=1_{v}$.

One can then compose paths in $\Gamma$ in the usual way. If $p_{1} \omega=p_{2} \alpha$, then $p_{1} p_{2}$ is obtained by juxtaposition of the edge sequences. If $p_{1}$ and $p_{2}$ are directed, so is $p_{1} p_{2}$. The product of two loops at a vertex is evidently another loop. Composition is associative and the empty paths act as local identities so we get a category $\Pi(\Gamma)$ with vertex set $V(\Gamma)$ and hom sets $\Pi(\Gamma)\left(v_{1}, v_{2}\right)=$ paths from $v_{1}$ to $\left.v_{2}\right\}$. Note that $\Pi(\Gamma)$ is a category with an involution operation $p \mapsto \bar{p}$. If $\Gamma$ is oriented, then $\Gamma^{*}$ will denote the subcategory of $\Pi(\Gamma)$ consisting of all directed paths and is what is usually referred to as the free category generated by the directed graph $\Gamma$.

We say that $\Gamma$ is connected if any two vertices can be joined by a path, while we say that an oriented graph $\Gamma$ is strongly connected if this can be done by a directed path. A connected component of a graph is a maximal connected subgraph. Any graph is a disjoint union of its connected components; hence we shall mostly consider connected graphs. A maximal strongly connected subgraph of an oriented graph is called a strongly connected component. In an oriented graph $\Gamma$, we write, for $v, w \in V(\Gamma), w \leq v$ (read $w$ is accessible from $v$ ), if there is a directed path from $v$ to $w$. This is a preorder and if $\equiv$ is the associated equivalence relation, then $v \equiv w$ if and only if they are in the same strongly connected component of $\Gamma$.

3.2. The Fundamental Group of a Graph. As this material is well known, see [39] 40, we merely give the definitions and state the primary result. Let $\Gamma$ be a graph. For $p_{1}, p_{2} \in \Pi(\Gamma)\left(v_{1}, v_{2}\right)$ we say $p_{1} \sim p_{2}$ (read $p_{1}$ is homotopic to $\left.p_{2}\right)$ if $p_{1}$ can be obtained from $p_{2}$ by deletions and insertions of paths of the form $e \bar{e}$. This is easily seen to be a congruence and the resulting quotient $\pi(\Gamma)$ is a groupoid called the fundamental groupoid of $\Gamma$. The equivalence class $[p]$ of a path $p$ is called the homotopy class of $p$. The inverse of a path $[p]$ is $[\bar{p}]$. If $v \in V(\Gamma)$, then $\pi_{1}(\Gamma, v)=\pi(\Gamma)(v, v)$ is called the fundamental group of $\Gamma$ at $v$. If $v$ and $v^{\prime}$ are in 
the same connected component of $\Gamma$, then they have (non-canonically) isomorphic fundamental groups.

A path $p \in \Pi(\Gamma)$ is said to be reduced if it has no subpath of the form $e \bar{e}$. For instance, any directed path in an oriented graph is reduced. One can show that each homotopy class of $\pi(\Gamma)$ contains a unique reduced path. We shall not, in general, distinguish between a reduced path $p$ and its homotopy class. A connected graph $\Gamma$ is called a tree if the only reduced circuits in $\Gamma$ have length 0 , that is $\pi_{1}(\Gamma, v)=1$ for every vertex $v$. By Zorn's lemma, every connected graph has a maximal (sub)tree, and this maximal tree contains every vertex. The following theorem is at the root of combinatorial group theory; see, for instance, [40].

Theorem 3.1. Let $\Gamma$ be a connected oriented graph, $T \subseteq \Gamma$ be a maximal subtree, and $v_{0} \in V(\Gamma)$. For each $v \in V(\Gamma)$, let $p_{v}$ be the unique reduced path in $T$ from $v_{0}$ to $v$. Define for $e \in E^{+}(\Gamma), \tilde{e}=p_{e \alpha} e \overline{p_{e \omega}}$. Then $\pi_{1}\left(\Gamma, v_{0}\right)$ is a free group generated by $\{[\tilde{e}] \mid e \notin E(T)\}$.

If $\Gamma$ is finite, one can algorithmically (in polynomial time) find a basis for the fundamental group of $\Gamma$ at any vertex by first constructing a maximal tree $T$, and then computing the appropriate $\tilde{e}$.

3.3. Graph Morphisms and Immersions. A graph morphism $\varphi: \Gamma \rightarrow \Delta$ consists of a pair of functions $\varphi_{V}: V(\Gamma) \rightarrow V(\Delta)$ and $\varphi_{E}: E(\Gamma) \rightarrow E(\Delta)$; however, it will be convenient to just use $\varphi$ for both these functions. The requirements on a graph morphism are: $e \varphi \alpha=e \alpha \varphi$ and $\bar{e} \varphi=\overline{e \varphi}$. If we let $A_{n}$ be the standard arc $[0, n]$, subdivided at the integer points (where there is a positively oriented edge from $j$ to $j+1$ ), then a path $p$ of length $n$ from $v_{1}$ to $v_{2}$ corresponds to a graph morphism $p: A_{n} \rightarrow \Gamma$, explaining our conventions on empty paths. One can easily show that $\Pi$ and $\pi$ are functors (if we look at the category of graphs with base points and base point preserving graph morphisms, then $\pi_{1}$ is a functor as well). If we view a path $p$ of length $n$ as a graph morphism $p: A_{n} \rightarrow \Gamma$, then $p \Pi(\varphi)=p \varphi$ leading us to just write $\varphi$ in place of $\Pi(\varphi)$, and similarly for $\pi(\varphi)$. If $\Gamma$ and $\Delta$ are oriented, $\varphi$ is called orientation-preserving if it sends positively oriented edges to positively oriented edges. In the category of oriented graphs and orientationpreserving maps $\Gamma \mapsto \Gamma^{*}$ is a functor. Note that a directed path $p$ of length $n$ is an orientation-preserving graph morphism $p: A_{n} \rightarrow \Gamma$. If $\varphi: \Gamma \rightarrow \Delta$ is a graph morphism and if $\Delta$ is oriented, then there is a unique orientation of $\Gamma$ so that $\varphi$ is orientation-preserving called the orientation of $\Gamma$ induced by $\varphi$.

The following lemma is an easy exercise which we leave to the reader.

Lemma 3.2. Let $\Gamma_{1}, \Gamma_{2}$, and $\Delta$ be oriented graphs and

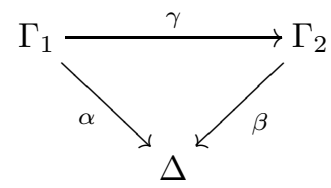

be a commutative diagram of graph morphisms. Then if $\gamma, \beta$ preserve orientation, so does $\alpha$. If $\alpha$ and $\beta$ preserve orientation, so does $\gamma$.

Hence if we have a fixed oriented graph $\Delta$ and consider the comma category of graphs with morphisms into $\Delta$, then if we orient all these graphs so that their 
morphisms to $\Delta$ preserve orientation, all the morphisms in this comma category become orientation-preserving.

We call a graph morphism $\varphi$ faithful if $e_{1} \alpha=e_{2} \alpha, e_{1} \omega=e_{2} \omega$, and $e_{1} \varphi=e_{2} \varphi$ imply that $e_{1}=e_{2}$. Let $\Gamma$ be a graph and $v \in V(\Gamma)$. Then we define $\operatorname{Star}(v)=$ $v \alpha^{-1}$. If $\varphi: \Gamma \rightarrow \Delta$ is a graph morphism, then for any vertex $v \in V(\Gamma)$, we have an induced map $\varphi_{v}: \operatorname{Star}(v) \rightarrow \operatorname{Star}(v \varphi)$. Following Stallings [40, $\varphi$ is called an immersion if $\varphi_{v}$ is injective, locally surjective if $\varphi_{v}$ is surjective, and a covering if $\varphi_{v}$ is bijective for all $v \in V(\Gamma)$. It is easy to see that a composition of maps with any of the above properties has that property as well. By way of example, a reduced path $p$ of length $n$ in a graph $\Gamma$ is an immersion $p: A_{n} \rightarrow \Gamma$.

The following elementary results are sketched in 40.

Theorem 3.3. Let $\varphi: \Gamma \rightarrow \Delta$ be an immersion and $v \in V(\Gamma)$.

1. $\varphi: \pi_{1}(\Gamma, v) \rightarrow \pi_{1}(\Delta, v \varphi)$ is injective.

2. If $p$ is a reduced path in $\Gamma$, then $p \varphi$ is a reduced path in $\Delta$.

3. If $p$ and $q$ are paths starting at $v$ and $p \varphi=q \varphi$, then $p=q$.

4. If $\varphi$ is a covering and $p$ a path starting at $v \varphi$, then there is a (unique) path $\tilde{p}$ starting at $v$ with $\tilde{p} \varphi=p$.

5. Suppose $\tau: \Gamma^{\prime} \rightarrow \Delta$ is a graph morphism with $\Gamma^{\prime}$ connected, $u \in V\left(\Gamma^{\prime}\right)$, and $u \tau=v \varphi$. Then the existence of $\tilde{\tau}: \Gamma^{\prime} \rightarrow \Gamma$ lifting $\tau$ (that is, $\tilde{\tau} \varphi=\tau$ ) with $u \tilde{\tau}=v$ implies $\pi_{1}\left(\Gamma^{\prime}, u\right) \tau \subseteq \pi_{1}(\Gamma, v) \varphi$; furthermore, $\tilde{\tau}$ is unique. If $\varphi$ is a covering, the above condition is sufficient.

We now state the standard theorem on the existence of coverings; see [40].

Theorem 3.4. If $\Delta$ is a connected graph, $v \in V(\Delta)$, and $H \subseteq \pi_{1}(\Delta, v)$ a subgroup, then there exists a covering $\varphi: \Gamma \rightarrow \Delta$ with $\Gamma$ connected, a vertex $u \in V(\Gamma)$ with $u \varphi=v$, and $\pi_{1}(\Gamma, u) \varphi=H$. Any two such coverings are isomorphic as based graphs over $(\Delta, v)$. The number of sheets of the covering, $\left|v \varphi^{-1}\right|$, is the index of $H$.

If $\Gamma$ is a graph, we say a graph morphism $\varphi: \Gamma \rightarrow \Delta$ is a fold if there exist edges $e_{1}, e_{2} \in E(\Gamma)$ such that $e_{1} \alpha=e_{2} \alpha, e_{2} \neq \overline{e_{1}}$, and $\Delta$ is obtained from $\Gamma$ by identifying these edges.

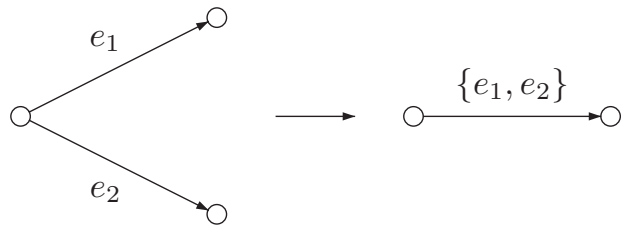

Stallings proves in [40] that a fold induces a surjective map on fundamental groups. The following observation of Stallings [40] will be important in the sequel.

Proposition 3.5. Let $\Gamma$ be a finite graph and $\varphi: \Gamma \rightarrow \Delta$ a morphism of graphs. Then $\varphi$ factors as a finite sequence of folds followed by an immersion. The sequence of folds need not be unique, but the immersion is unique.

The idea is that if $\varphi$ is not an immersion, then there exists a vertex $v$ and distinct edges $e_{1}, e_{2} \in \operatorname{Star}(v)$, with $e_{2} \neq \overline{e_{1}}$, such that $e_{1} \varphi=e_{2} \varphi$. One folds these two edges and repeats this procedure on the quotient graph. Hence one can construct this immersion in rapid polynomial time. 
3.4. Labeled Graphs. Let $A$ be a set. Then we use $B_{A}$ to denote a bouquet of $|A|$ circles; that is, $V\left(B_{A}\right)=\{1\}$ and $E\left(B_{A}\right)=\tilde{A}$ where $\bar{a}=a^{-1}$ for $a \in \tilde{A}$. We orient $B_{A}$ by choosing $E^{+}\left(B_{A}\right)=A$. One can then see that $B_{(}$is a functor which is right adjoint to the functor $E^{+}$. It is easy to see that $\Pi\left(B_{A}\right)=\tilde{A}^{*}, \pi\left(B_{A}\right)=F G(A)$, $B_{A}^{*}=A^{*}$ where we view each of these monoids as a one vertex category. Using Theorems 3.1, 3.3, and 3.4 and using that $\pi_{1}\left(B_{A}, 1\right)=F G(A)$, one obtains the well known result that any subgroup of a free group is free.

A labeling of a graph $\Gamma$ over $A$ consists of a faithful graph morphism $\ell: \Gamma \rightarrow B_{A}$; we orient $\Gamma$ by $\ell$. If $e$ is an edge, we call $e \ell$ the label of $e$. More generally, since there is an induced map $\ell: \Pi(\Gamma) \rightarrow \tilde{A}^{*}$, for a path $p$, we call $p \ell$ the label of $p$. If $p$ is directed, then $p \ell \in A^{*}$. Also note that if $p \ell$ is a reduced word, then $p$ cannot contain a factor $e \bar{e}$ whence $p$ is also reduced. We think of a labeled graph as a collection of vertices and oriented edges, each edge labeled by an element of $A$ and no two coterminal edges having the same label. We shall call a graph $\Gamma$ labeled over $A$ an A-graph.

A morphism $\varphi: \Gamma \rightarrow \Delta$ of A-graphs is a morphism in the comma category of graphs over $B_{A}$, that is, a graph morphism $\varphi$ such that

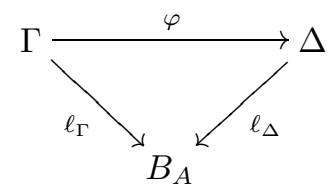

commutes. Note that, by Lemma 3.2 $\varphi$ automatically preserves orientation. We think of $\varphi$ as a label-preserving graph morphism.

A congruence $\sim$ on an $A$-graph $\Gamma$ is an equivalence relation $\sim$ on $V(\Gamma)$. The quotient $A$-graph $\Gamma / \sim$ is defined as follows: $V(\Gamma / \sim)=V(\Gamma) / \sim$; if $v, w \in V(\Gamma)$ and $[v],[w]$ are the $\sim$-classes of $v$ and $w$, respectively, then there is a positively oriented edge in $\Gamma / \sim,[v] \stackrel{a}{\longrightarrow}[w]$ with $a \in A$, if and only if there exist $v^{\prime} \in[v]$ and $w^{\prime} \in[w]$ such that there is an edge $v^{\prime} \stackrel{a}{\longrightarrow} w^{\prime}$ in $\Gamma$. Any morphism of $A$-graphs then factors through a quotient and an embedding.

Let $w \in \tilde{A}^{*}$. Then a run of $w$ in an $A$-graph $\Gamma$ is a path $p$ with $p \ell=w$. We say in this case that there is a run of $w$ from $p \alpha$ to $p w$. If $w \in \tilde{A}^{*}$, then the reversal of $w$ is the word $w^{\rho}$ obtained by reversing the order of the letters occurring in $w$, for instance $(a b)^{\rho}=b a$. A reverse run of a word $w \in \tilde{A}^{*}$ in $\Gamma$ from $p$ to $q$ is then a run of $w^{\rho}$ from $p$ to $q$.

Note that a word may have several runs from a vertex $p$ of an $A$-graph $\Gamma$; we would like to consider the situation where this does not occur. Let $\Gamma$ be an oriented graph. Define, for $v \in V(\Gamma), \operatorname{Star}^{+}(v)=\operatorname{Star}(v) \cap E^{+}(\Gamma)$ and $\operatorname{Star}^{-}(v)=$ $v \omega^{-1} \cap E^{+}(\Gamma)$. Let $\varphi: \Gamma \rightarrow \Delta$ be an orientation-preserving graph morphism. We call $\varphi$ deterministic if $\varphi$ is injective when restricted to $\operatorname{Star}^{+}(v)$ for all $v \in V(\Gamma)$ and codeterministic if $\varphi$ is injective when restricted to $\operatorname{Star}^{-}(v)$ for all $v \in V(\Gamma)$. Note that $\varphi$ is an immersion if and only if it is deterministic and codeterministic. If $\Gamma$ is an $A$-graph, we call $\Gamma$, respectively, deterministic, codeterministic, inverse if the labeling $\ell$ is, respectively, deterministic, codeterministic, an immersion. If $\ell$ is locally surjective, $\Gamma$ is called complete; if $\ell$ is a covering, $\Gamma$ is called a cover.

If $\Gamma$ is a deterministic $A$-graph, then one can show inductively that each $w \in A^{*}$ has at most one run from any vertex $q \in V(\Gamma)$. We let $q w=p$ if the run of $w$ from $q$ exists and ends at $p$, and leave it undefined otherwise. Each $w \in A^{*}$ 
defines, in this manner, a partial function on $V(\Gamma)$. Furthermore, for $w_{1}, w_{2} \in A^{*}$, $\left(q w_{1}\right) w_{2}=q\left(w_{1} w_{2}\right)$, and hence we have a well defined homomorphism of $A^{*}$ into the monoid of partial functions on $V(\Gamma)$. The image is called the transition monoid of $\Gamma$ and is denoted $M(\Gamma)$. Analogously, each word $w \in A^{*}$ has at most one reverse run from any vertex. We let $w q=p$ if the reverse run of $w$ from $q$ ends at $p$, and leave $w q$ undefined otherwise. In this way, we again obtain a set of partial function on $V(\Gamma)$, this time acting on the left. As before, these partial functions form a monoid $M^{\rho}(\Gamma)$ called the left transition monoid of $\Gamma$. If $M$ is a monoid, we use $M^{\rho}$ to denote the reverse monoid; it is then easy to see that $M^{\rho}(\Gamma)=M(\Gamma)^{\rho}$. Hence we will usually leave it to the reader to supply dual results and proofs for reverse runs and left transition monoids. Note that $M(\Gamma)$ consists of functions if and only if $\Gamma$ is complete.

A deterministic $A$-graph congruence on a labeled $A$-graph $\Gamma$ is an $A$-graph congruence $\sim$ such that for $p, q, p^{\prime}, q^{\prime} \in V(\Gamma)$ if $p a=q, p^{\prime} a=q^{\prime}$, and $p \sim p^{\prime}$, then $q \sim q^{\prime}$. The quotient $\Gamma / \sim$ is then a deterministic $A$-graph and any morphism of $\Gamma$ to a deterministic $A$-graph, factors through a quotient by a deterministic $A$-graph congruence followed by an embedding.

If $\Gamma$ is an inverse $A$-graph, that is, the labeling is an immersion, then each $w \in \tilde{A}^{*}$ has at most one run from any $q \in V(\Gamma)$. Again, let $q w=p$ if $w$ has a run from $q$ to $p$, and leave it undefined otherwise. Then $w$ gives a partial one-to-one function with inverse $w^{-1}$. At this point, to avoid confusion, if $\Gamma$ is an $A$-graph with the labeling an immersion, we write $\mathcal{I}(\Gamma)$ if we want to think of $\Gamma$ as an inverse $A$ graph while we shall just write $\Gamma$ when we only want to think of $\Gamma$ as an $A$-graph. Then the transition monoid of $\mathcal{I}(\Gamma)$, denoted $M(\mathcal{I}(\Gamma))$, is the monoid of partial one-to-one functions induced by the various $w \in \tilde{A}^{*}$. We note that $M(\Gamma)$ consists of partial one-to-one functions and is a submonoid of $M(\mathcal{I}(\Gamma))$. In fact, $M(\mathcal{I}(\Gamma))$ is the inversification of $M(\Gamma)$, obtained by adding to $M(\Gamma)$ the inverse of each element. We note that $\mathcal{I}(\Gamma)$ is complete if and only if $\Gamma$ is a cover; this occurs if and only if $M(\mathcal{I}(\Gamma))$ is a group. If $\Gamma$ is finite, $\mathcal{I}(\Gamma)$ is a cover if and only if $M(\Gamma)$ is a group in which case $M(\Gamma)=M(\mathcal{I}(\Gamma))$. In an inverse $A$-graph, it is easy to see that a path $p$ is reduced if and only if $p \ell$ is reduced. Indeed from our earlier remarks, it suffices to show that if $p$ is reduced then so is $p \ell$. But immersions take reduced paths to reduced paths. Note that the fundamental group of an inverse $A$-graph is a subgroup of $F G(A)$. The following observation will be of frequent use.

Proposition 3.6. Let $\Gamma$ be an inverse A-graph and $v \in V(\Gamma)$. Suppose $p, q \in V(\Gamma)$ and $w_{p}, w_{q} \in \tilde{A}^{*}$ label reduced paths from $v$ to $p, q$ respectively. Then $p=q$ if and only if $w_{p} w_{q}^{-1} \in \pi_{1}(\Gamma, v)$.

Proof. Clearly if $p=q$, then $w_{p} w_{q}^{-1} \in \pi_{1}(\Gamma, v)$. For the converse, suppose $u \in \tilde{A}^{*}$ is the largest common suffix of $w_{p}$ and $w_{q}$ and that $w_{p}=u_{p} u$ and $w_{q}=u_{q} u$. Then, since $w_{p} w_{q}^{-1}=u_{p} u_{q}^{-1}$ in $\pi_{1}(\Gamma, v)$ and the right-hand side is reduced, the run of $u_{p} u_{q}^{-1}$ at $v$ exists and is a loop. Since $\Gamma$ is inverse, it follows that $v u_{p}=v u_{q}$ whence

$$
p=v w_{p}=v u_{p} u=v u_{q} u=v w_{q}=q .
$$

At this point, we briefly pause to introduce some semigroup theoretic concepts. An element $m$ of a monoid $M$ is called regular if $m \in m M m$. An element $e \in M$ is called idempotent if $e^{2}=e$. We shall write $E(M)$ for the set of idempotents of $M$. 
Elements $m$ and $n$ are called inverses if $m n m=m$ and $n m n=n$. Every regular element has at least one inverse. If $m n m=m$, then $m n$ and $n m$ are idempotents. A monoid $M$ is called regular if every element of $M$ is regular, a block group if each regular element of $M$ has a unique inverse, and inverse if it is a regular block group. Equivalently, a monoid is inverse if it is regular and its idempotents commute. For example, any group is an inverse monoid.

If $I$ is an inverse monoid, we write $m^{-1}$ for the inverse of $m \in I$. Then $\left(m^{-1}\right)^{-1}=$ $m$ and $(m n)^{-1}=n^{-1} m^{-1}$. Hence any inverse monoid is isomorphic to its reversal. Inverse monoids can be viewed as unary monoids and form a variety of such defined by the equations $x x^{-1} x=x,\left(x^{-1}\right)^{-1}=x$, and $x x^{-1} y y^{-1}=y y^{-1} x x^{-1}$. Thus there is a free inverse monoid on any set $A$, which we denote by $F I M(A)$. Note that the canonical projection of $\tilde{A}^{*}$ to $F G(A)$ factors through $F I M(A)$ with the property that the inverse image of 1 in $F I M(A)$ is $E(F I M(A))$. The word problem for $F I M(A)$ has a beautiful geometric solution, due to Munn [32, which we shall discuss later. If $I$ is an inverse monoid such that $E(I)=I$, that is an idempotent commutative monoid, then $I$ is called a semilattice. Semilattices form a variety of monoids and it is easy to see that the free semilattice on a finite set is again finite. Note that, for any inverse monoid $I, E(I)$ is a semilattice. We remark that, for an inverse $A$-graph $\Gamma, M(\mathcal{I}(\Gamma))$ is an inverse monoid. Indeed, the inverse of the partial function induced by $w$ is the one induced by $w^{-1}$.

An inverse $A$-graph congruence on an $A$-graph $\Gamma$ is a deterministic $A$-graph congruence with the additional property that, for $a \in A, p, q, p^{\prime}, q^{\prime} \in V(\Gamma)$, if $p a=q, p^{\prime} a=q^{\prime}$, and $q \sim q^{\prime}$, then $p \sim p^{\prime}$. The quotient $\Gamma / \sim$ is then an inverse $A$-graph and any morphism of $\Gamma$ to an inverse $A$-graph factors, through a quotient by such a congruence followed by an embedding.

Note that if $\Gamma$ is an oriented graph, then there is a natural immersion $\ell: \Gamma \rightarrow$ $B_{E^{+}(\Gamma)}$, and so every graph can be made an inverse $A$-graph for some set $A$.

3.5. Automata. Let $A$ be a finite set (one often calls such a set an alphabet). Then a non-deterministic A-automaton is a pair $\left(\Gamma, q_{0}\right)$ with $\Gamma$ a finite, connected $A$-graph and $q_{0} \in V(\Gamma)$. We shall often call $V(\Gamma)$ the state set, vertices states, and $q_{0}$ the initial state. In the literature, one often requires a subset $F \subseteq V(\Gamma)$, called the set of final states, as part of the data for such an automaton, but as we are interested in the geometric and algebraic structure of the automaton, we do not require this extra information. We call a non-deterministic automaton accessible if every state is accessible from the initial state. A strongly connected, non-deterministic automaton is one whose underlying graph is strongly connected. These will be our primary object of study. Often, we shall just denote the non-deterministic automaton by $\Gamma$ if the initial state is clear from context, or if we are not so interested in it. An automaton morphism is a morphism of labeled graphs preserving initial states. A non-deterministic automaton congruence is an A-graph congruence. The initial state of the quotient is then the equivalence class of the initial state.

A deterministic A-automaton, or, more simply, an A-automaton, is a non-deterministic $A$-automaton $\left(\Gamma, q_{0}\right)$ such that $\Gamma$ is a deterministic $A$-graph. We call $M(\Gamma)$ the transition monoid of the automaton. An automaton is called complete if $\Gamma$ is a complete $A$-graph. One defines an automaton congruence to be a deterministic $A$-graph congruence. The initial state of the quotient automaton is the equivalence class of the initial state of the original automaton. 
An inverse $A$-automaton is an $A$-automaton $\left(\Gamma, q_{0}\right)$ with $\Gamma$ an inverse $A$-graph. We shall use $\left(\mathcal{I}(\Gamma), q_{0}\right)$ or $\mathcal{I}\left(\Gamma, q_{0}\right)$ to distinguish between $\left(\Gamma, q_{0}\right)$ viewed as an $A$ automaton and an inverse $A$-automaton. The transition monoid of the inverse automaton is then $M(\mathcal{I}(\Gamma))$. Again, we shall often just write $\mathcal{I}(\Gamma)$ if $q_{0}$ is understood or not of significance. An inverse automaton congruence is an inverse $A$-graph congruence and the quotient is made an inverse automaton by choosing the class of the initial state as the initial state of the quotient.

3.6. An Important Functor. Let $A$ be a set. We use $\mathcal{G}_{A}$ for the category of $A$-graphs and $\mathcal{I}_{A}$ for the category of inverse $A$-graphs. Observe that the forgetful functor $\mathcal{I}(\Gamma) \mapsto \Gamma$ embeds $\mathcal{I}_{A}$ as a full subcategory of $\mathcal{G}_{A}$. We now construct a left adjoint $\mathcal{I}: \mathcal{G}_{A} \rightarrow \mathcal{I}_{A}$ such that $\mathcal{I}(\Gamma)$, for an inverse $A$-graph $\Gamma$, has the same meaning as before. We shall call this functor inversification as it generalizes the previous sense of the term.

Let $\Gamma \in \mathcal{G}_{A}$. We define a congruence $\sim_{\Gamma}$ on $\Gamma$ such that the map $\varphi_{\Gamma}: \Gamma \rightarrow \Gamma / \sim_{\Gamma}$ is universal for labeled graph morphisms of $\Gamma$ into inverse $A$-graphs. Then letting $\mathcal{I}(\Gamma)=\mathcal{I}\left(\Gamma / \sim_{\Gamma}\right)$ (where the right-hand side is in the original sense) will give the left adjoint. We note that if $\Gamma$ is an inverse $A$-graph, then the identity map is clearly universal and so the notation $\mathcal{I}(\Gamma)$ is unambiguous.

Define, for $p, q \in V(\Gamma), p \sim_{\Gamma} q$ if there exists $w \in \tilde{A}^{*}$ such that $w$ has a run from $p$ to $q$ and $[w]_{F G(A)}=1$. Using that the set

$$
K=\left\{w \in \tilde{A}^{*} \mid[w]_{F G(A)}=1\right\}
$$

is a unary submonoid of $\tilde{A}^{*}$, it is easy to show that $\sim_{\Gamma}$ is an equivalence relation. We show that $\sim_{\Gamma}$ is deterministic. Suppose $p \sim_{\Gamma} p^{\prime}$ and $p a=q, p^{\prime} a=q^{\prime}$. Let $w \in K$ such that $w$ runs from $p$ to $p^{\prime}$. Then $a^{-1} w a$ runs from $q$ to $q^{\prime}$, so $q \sim_{\Gamma} q^{\prime}$. A dual verification shows that $\sim_{\Gamma}$ is an inverse $A$-graph congruence whence $\Gamma / \sim_{\Gamma}$ is an inverse $A$-graph. Suppose $\Delta$ is an inverse $A$-graph and $\varphi: \Gamma \rightarrow \Delta$ is a morphism of $A$-graphs; we show that $\varphi$ factors through $\varphi_{\Gamma}$. First, we shall need the following standard lemma which can be proved by induction.

Lemma 3.7. One has that $K$ is the smallest subset of $\tilde{A}^{*}$ such that:

1. $1 \in K$;

2. $u, v \in K$, implies $u v \in K$;

3. $u \in K, a \in \tilde{A}$, implies aua ${ }^{-1} \in K$.

We now prove, by induction on $|w|$, that if $w \in K$ and $w$ has a run from $p$ to $q$ in $\Gamma$, then $p \varphi=q \varphi$; it will then follow that $\varphi$ factors through $\varphi_{\Gamma}$. Suppose first that $|w|=0$; then $p=q$, so $p \varphi=q \varphi$. Suppose now that $w=u v$ with $u, v \in K$, $|u|,|v|<|w|$, and that $w$ has a run from $p$ to $q$. Let $p^{\prime}$ be the vertex reached on reading $u$ in this run. Then by induction, $p \varphi=p^{\prime} \varphi=q \varphi$. Suppose now that $w=a u a^{-1}$ with $a \in \tilde{A}, u \in K$ and that $w$ has a run from $p$ to $q$; then $|u|<|w|$. Let $p^{\prime}$ be the vertex reached after reading the first $a$ in this run and $q^{\prime}$ the vertex from which the last $a^{-1}$ is read. Then $u$ reads from $p^{\prime}$ to $q^{\prime}$ so, by induction, $p^{\prime} \varphi=q^{\prime} \varphi$. Now, since $\Delta$ is inverse and $\varphi$ a morphism, $p \varphi=p^{\prime} \varphi a^{-1}, q \varphi=q^{\prime} \varphi a^{-1}$ whence $p \varphi=q \varphi$. We have thus proved the following theorem.

Theorem 3.8. Let $\mathcal{I}: \mathcal{G}_{A} \rightarrow \mathcal{I}_{A}$ be given by $\mathcal{I}(\Gamma)=\mathcal{I}\left(\Gamma / \sim_{\Gamma}\right)$ (in the previous sense on the right-hand side). Then $\mathcal{I}$ is left adjoint to the forgetful functor. 
Suppose $\Gamma$ is a finite $A$-graph. We now give a geometric description of $\mathcal{I}(\Gamma)$ which also shows that it is constructible in polynomial time. Indeed, recall from Proposition 3.5 that $\ell: \Gamma \rightarrow B_{A}$ factors through a finite sequence of folds and an immersion $\ell^{\prime}: \Gamma^{\prime} \rightarrow B_{A}$. The sequence need not be unique, but the immersion is unique. Also one can perform these folds in polynomial time. We show that $\mathcal{I}(\Gamma)=\Gamma^{\prime}$. To do this, it suffices to show that the map to $\Gamma^{\prime}$ has the desired universal property.

Proposition 3.9. Let $\Gamma$ and $\Gamma^{\prime}$ be as above and let $\varphi: \Gamma \rightarrow \Delta$ be a labeled graph morphism with $\Delta$ an inverse A-graph. Then $\varphi$ factors through the quotient map from $\Gamma$ to $\Gamma^{\prime}$.

Proof. The morphism $\varphi$ factors through a finite sequence folds and an immersion $\psi: \Delta^{\prime} \rightarrow \Delta$. But then if $\ell_{\Delta}: \Delta \rightarrow B_{A}$ is the labeling, $\psi \ell_{\Delta}$ is an immersion. Since $\ell=\varphi \ell_{\Delta}$ factors through a sequence of folds and $\psi \ell_{\Delta}$, we see, by the uniqueness of the immersion in Proposition 3.5, that $\Delta^{\prime}=\Gamma^{\prime}$ and the result follows.

For a non-deterministic $A$-automaton $\left(\Gamma, v_{0}\right)$, we shall denote $\left(\mathcal{I}(\Gamma),\left[v_{0}\right]\right)$ by $\mathcal{I}\left(\Gamma, v_{0}\right)$.

\section{Monoid And Semigroup Theory}

We are finally ready to begin applying the machinery we have been building to the theory of monoids. As mentioned earlier, we shall generally leave to the reader semigroup theoretic analogs of our definitions and results.

4.1. Cayley and Schützenberger Graphs. Let $M$ be an $A$-generated monoid. We then define $\Gamma_{A}(M)$, the right Cayley graph of $M$ with respect to $A$, to be the complete deterministic $A$-graph with $V\left(\Gamma_{A}(M)\right)=M$ and $E^{+}\left(\Gamma_{A}(M)\right)=M \times A$. We let $(m, a) \alpha=m,(m, a) \omega=m[a]_{M}$, and the label of $(m, a)$ be $a$. Normally, we draw the edge $(m, a)$ as

$$
m \stackrel{a}{\longrightarrow} m[a]_{M} .
$$

Note that $M\left(\Gamma_{A}(M)\right)=M$. The left Cayley graph of $M$ with respect to $A, \Gamma_{A}^{\rho}(M)$ is the complete deterministic $A$-graph with $V\left(\Gamma_{A}^{\rho}(M)\right)=M, E^{+}\left(\Gamma_{A}^{\rho}(M)\right)=M \times A$, $(m, a) \alpha=m$, and $a$ the label of $(m, a)$, but now with $(m, a) \omega=[a]_{M} m$. Evidently, $M^{\rho}\left(\Gamma_{A}^{\rho}(M)\right)=M$. In fact, $\Gamma_{A}^{\rho}(M)=\Gamma_{A}\left(M^{\rho}\right)$.

If $m, n \in M$, we say that $m \mathcal{R} n$ (read $m$ is $\mathcal{R}$-equivalent to $n)$ if $m$ and $n$ are in the same strongly connected component of $\Gamma_{A}(M)$. We denote the $\mathcal{R}$-class of $m$, $R_{m}$. More generally, we write $m \leq_{\mathcal{R}} n(\operatorname{read} m$ is $\mathcal{R}$-below $n)$ if $m \leq n$ in $\Gamma_{A}(M)$. These notions are independent of $A$ since it is easy to see that $m \leq_{\mathcal{R}} n$ if and only if $m M \subseteq n M$. Similarly, we say $m \mathcal{L} n$, respectively, $m \leq_{\mathcal{L}} n$ if in $\Gamma_{A}^{\rho}(M), m \equiv n$, respectively, $m \leq n$. Finally, we say that for $m, n \in M, m \mathcal{J} n$, respectively, $m \leq \mathcal{J} n$ if $M m M=M n M$, respectively, $M m M \subseteq M n M$. An $\mathcal{R}$-class is called regular if it contains an idempotent. It can be shown that an $\mathcal{R}$-class is regular if and only if each element of the $\mathcal{R}$-class is regular. Dual remarks apply to $\mathcal{L}$-classes and we continue to leave such dualizations to the reader. We refer the reader to [12] for more on the fundamentals of monoid theory.

If $X$ is an $\mathcal{R}$-class of $M$, the Schützenberger graph (with respect to $A$ ) of $X$, denoted $\operatorname{Sch}_{A}(X)$, is the full subgraph of $\Gamma_{A}(M)$ with vertices $X$. We note that $\operatorname{Sch}_{A}(X)$ is a strongly connected, deterministic $A$-graph. We call $M\left(S c h_{A}(X)\right)$ the Schützenberger representation of $X$ and denote it by $S \operatorname{ch}(X)$. Observe that 
this monoid is independent of $A$ and can be described as the monoid of partial functions on $X$ arising from right multiplication by elements of $M$. If $m \mathcal{L} n$ and $r n=m$, it can be shown that left multiplication by $r$ induces a graph isomorphism of Schützenberger graphs. Hence, up to isomorphism, each $\mathcal{D}$-class of $M$ has exactly one corresponding Schützenberger graph (the relation $\mathcal{D}$ is the join of $\mathcal{R}$ and $\mathcal{L}$ and, for finite monoids, $\mathcal{D}=\mathcal{J})$. Dually, for an $\mathcal{L}$-class $Y$, we use the notation $\operatorname{Sch}_{A}^{\rho}(Y)$ for the left Schützenberger graph and $S_{c h}{ }^{\rho}(Y)$ for the left Schützenberger representation of $Y$. That is, $S c h^{\rho}(Y)=M^{\rho}\left(S c h_{A}^{\rho}(Y)\right)$. To each $\mathcal{D}$-class there is also only one left Schützenberger graph up to isomorphism.

If $M$ and $A$ are finite, then $\left(\Gamma_{A}(M), 1\right)$ is an accessible $A$-automaton. For a regular $\mathcal{R}$-class $X$, we shall usually make $S c h_{A}(X)$ an $A$-automaton by choosing an idempotent as the initial state. In the cases of most interest to us, there will be only one idempotent to choose from.

If $I$ is an inverse monoid generated by $A$ (as an inverse monoid) and $X$ is an $\mathcal{R}$-class, we define the Schützenberger graph of $X, \mathcal{I}\left(S c h_{A}(X)\right)$, to be the graph with vertex set $X$ and set of positively oriented edges

$$
\left\{(x, a) \in X \times A \mid x[a]_{I} \in X\right\} .
$$

One defines $(x, a) \alpha=x,(x, a) \omega=x[a]_{I}$, and the label of $(x, a)$ to be $a$. If $I$ and $A$ are finite, we choose the unique idempotent of $\mathcal{R}$ to be the initial state (since $E(I)$ is a semilattice, one can check that an $\mathcal{R}$-class has a unique idempotent). We note that $M\left(\mathcal{I}\left(S c h{ }_{A}(X)\right)\right)=S c h(X)$ and hence is independent of the generating set. Of course, we should justify the notation $\mathcal{I}\left(S c h_{A}(X)\right)$.

Proposition 4.1. Let $X$ be an $\mathcal{R}$-class of an inverse monoid I. Then $\mathcal{I}\left(S c h_{A}(X)\right)$ is an inverse A-graph.

Proof. We show that if $x, y \in I$ and $x \mathcal{R} x y$ then $x y y^{-1}=x$. It then follows that $\operatorname{Sch}(X)$ consists of partial one-to-one maps on $X$. So suppose $x y t=x$ with $t \in I$. Then

$$
\begin{aligned}
x^{-1}\left(x y y^{-1}\right) x^{-1} & =\left(t^{-1} y^{-1} x^{-1}\right)\left(x y y^{-1}\right) x^{-1} \\
& =t^{-1} y^{-1} y y^{-1} x^{-1} x x^{-1}=t^{-1} y^{-1} x^{-1}=x^{-1} .
\end{aligned}
$$

On the other hand,

$$
\left(x y y^{-1}\right) x^{-1}\left(x y y^{-1}\right)=x x^{-1} x y y^{-1} y y^{-1}=x y y^{-1} .
$$

Thus $x^{-1}$ and $x y y^{-1}$ are inverses whence $x=x y y^{-1}$.

Observe that since an inverse monoid is isomorphic to its reversal, there is no reason to study its left Schützenberger graphs. Note that, for a group $G$ generated by $A$ as a group, one can define its Cayley graph $\Gamma_{A}(G)$ with respect to $A$ in the exact same manner (in fact, viewing $G$ as an inverse monoid, this is the Schützenberger graph of the unique $\mathcal{R}$-class of $G$ ) and the resulting graph is a cover (what is called in topology a regular cover). However, if $G$ is not a torsion group, then, in general, $G=M\left(\mathcal{I}\left(\Gamma_{A}(G)\right)\right) \neq M\left(\Gamma_{A}(G)\right)$. A torsion group is, of course, generated by $A$ as a group if and only if it is generated by $A$ as a monoid, and so there is no ambiguity.

4.2. Varieties and Pseudovarieties of Monoids. We note that every definition given here for monoids has a semigroup, inverse monoid, and unary monoid analog which we shall use freely. The following notions, which may seem a bit strange to those not familiar with them, go back to Eilenberg, Krohn, Rhodes, and Tilson. 
If $M$ and $N$ are monoids, then a relational morphism $\varphi: M \leftrightarrow N$ is a relation $\varphi \subseteq M \times N$ which is a submonoid whose projection to $M$ is onto. If the projection to $N$ is injective, we say $\varphi$ is a division and that $M$ divides $N$ (or is a divisor of $N$ ). If $M$ and $N$ are both $A$-generated, then there is a natural relational morphism $\varphi: M \leftrightarrow N$ given by

$$
\left\langle\left([a]_{M},[a]_{N}\right)\right\rangle \subseteq M \times N .
$$

We call this the canonical relational morphism.

A variety of monoids is a class of monoids closed under taking arbitrary products and divisors. A well known theorem of Birkhoff states that varieties are defined by identities and have free objects on any set. A pseudovariety of monoids is a class of finite monoids closed under taking finite products and divisors. Examples include the classes: $\mathbf{M}$ of all finite monoids; $\mathbf{G}$ of all finite groups; $\mathbf{G}_{p}$ of all $p$-groups; $\mathbf{G}_{\text {sol }}$ of all finite solvable groups; and $\mathbf{G}_{n i l}$ of all finite nilpotent groups. An intersection of varieties, respectively, pseudovarieties is again a variety, respectively, pseudovariety, so the classes of varieties and pseudovarieties form complete lattices. If $\mathbf{V}$ and $\mathbf{W}$ are varieties, respectively, pseudovarieties, the least variety, respectively, pseudovariety containing them both is called their join and is denoted $\mathbf{V} \vee \mathbf{W}$.

If $\varphi: M \hookrightarrow N$ is a relational morphism of finite monoids, then, for $e \in E(N)$, $e \varphi^{-1}$ is a semigroup. We define the Mal'cev kernel of $\varphi$ to be the pseudovariety of semigroups $\mathbf{V}_{\varphi}$ generated by $e \varphi^{-1}$ with $e \in E(N)$. If $\mathbf{V}$ is a pseudovariety of semigroups and $\mathbf{W}$ a pseudovariety of monoids, then the collection of all finite monoids $M$ with a relational morphism $\varphi: M \leftrightarrow N \in \mathbf{W}$ with Mal'cev kernel in $\mathbf{V}$ (that is, $\mathbf{V}_{\varphi} \subseteq \mathbf{V}$ ) forms a pseudovariety $\mathbf{V}(m \mathbf{W}$ called the Mal'cev product of $\mathbf{V}$ and $\mathbf{W}$. The Mal'cev product is not associative, but the following property holds; see, for instance, 50.

Proposition 4.2. Let $\mathbf{U}$ and $\mathbf{V}$ be pseudovarieties of semigroups and $\mathbf{W}$ a pseudovariety of monoids. Then

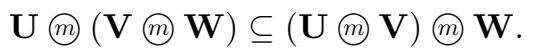

If $M$ and $N$ are monoids, a left action of $N$ on $M$ is a homomorphism of $N$ into the endomorphism monoid of $M$. We shall write the action by left exponentiation. The semidirect product of $M$ and $N$ with respect to this action, denoted $M * N$, is then $M \times N$ with multiplication given by

$$
(m, n)(r, s)=\left(m^{n} r, n s\right),
$$

exactly as for groups. It is easy to see that this is a monoid with identity $(1,1)$. If $\mathbf{V}$ and $\mathbf{W}$ are pseudovarieties of monoids, then $\mathbf{V} * \mathbf{W}$ is the collection of monoids which divide a semidirect product $M * N$ with $M \in \mathbf{V}$ and $N \in \mathbf{W}$. This forms a pseudovariety called the semidirect product of $\mathbf{V}$ and $\mathbf{W}$. The semidirect product operation is associative; see [14.

If $\varphi: M \leftrightarrow G$ is a relational morphism with $G$ a group, then $1 \varphi^{-1}$ is a submonoid of $M$. If $\mathbf{H}$ is a pseudovariety of groups (and we shall reserve $\mathbf{H}$ for such pseudovarieties) and $\mathbf{V}$ is a pseudovariety of monoids then it is easy to see that $\mathbf{V} * \mathbf{H} \subseteq \mathbf{V} m \mathbf{H}$. A sufficient condition for equality is that $\mathbf{V}$ is local in the sense of Tilson [49], see [24] for more. A monoid is called, respectively, $\mathcal{R}$-trivial, $\mathcal{L}$-trivial, $\mathcal{J}$-trivial if the corresponding relation is the equality relation. We let $\mathbf{R}, \mathbf{L}$, and $\mathbf{J}$ denote the respective pseudovarieties of finite $\mathcal{R}$-trivial, $\mathcal{L}$-trivial, and $\mathcal{J}$-trivial monoids. It turns out that $\mathbf{R}$ and $\mathbf{L}$ are local [14, 49], but $\mathbf{J}$ is not [16, 49]; thus 
$\mathbf{R} * \mathbf{H}=\mathbf{R} \rightarrow \mathbf{H}$ and $\mathbf{L} * \mathbf{H}=\mathbf{L} m \mathbf{H}$. In this paper, we shall study $\mathbf{V} m \mathbf{H}$ and $\mathbf{V} * \mathbf{H}$, particularly for these three pseudovarieties. The heart of the paper concerns the equation $\mathbf{J} * \mathbf{H}=\mathbf{J}: \mathbf{H}$; we shall give classes of pseudovarieties $\mathbf{H}$ for which the equation holds and classes for which it fails to hold as well as some conjectures on a complete classification.

Let $\mathbf{H}$ be a pseudovariety of groups and $M$ a finite monoid. Then the $\mathbf{H}$-kernel of $M$

$$
K_{\mathbf{H}}(M)=\bigcap_{\mu: M \rightarrow G \in \mathbf{H}} 1 \mu^{-1}
$$

is a submonoid of $M$ containing $E(M)$. Although, at first glance, this definition may look a bit arcane, we shall see that $K_{\mathbf{H}}(M)$ encodes a great deal of algebraic and geometric information about $M$. For instance, Proposition 4.3 will imply that $M \in$ $\mathbf{V} m \mathbf{H}$ if and only if $K_{\mathbf{H}}(M) \in \mathbf{V}$. We shall see later that $K_{\mathbf{H}}$ is a functor preserving surjection. We shall be interested in when this functor is effectively computable. The famous Rhodes type II conjecture, proved independently by Ash [10] and Ribes and Zalesskiı [37. (via a profinite group-theoretic argument), gives a polynomial time algorithm to compute $K_{\mathrm{G}}$. Ribes and Zalesskiu have given an algorithm to compute $K_{\mathbf{G}_{p}}$ [38] for $p$ a prime, shown recently to be polynomial time by Margolis, Sapir, and Weil [30]. We shall give rapid geometric algorithms to compute these kernels in this paper. It turns out that to understand $K_{\mathbf{H}}$, it is necessary to understand the pseudovariety of inverse monoids $\mathbf{S l} m \mathbf{H}$. This has been studied extensively in [30] and we give a new proof of their main result using our work on graphs and immersions.

We end this subsection with the following important proposition from [24].

Proposition 4.3. Let $M$ be a finite A-generated monoid and $\mathbf{H}$ a pseudovariety of groups. Then there exists an A-generated group $G \in \mathbf{H}$ such that if $\mu: M \hookrightarrow G$ is the canonical relational morphism, then $m \in K_{\mathbf{H}}(M)$ if and only if $m \in 1 \mu^{-1}$.

Proof. For each $m \in M \backslash K_{\mathbf{H}}(M)$, there exists $\varphi_{m}: M \multimap G_{m} \in \mathbf{H}$ such that $m \notin 1 \varphi_{m}^{-1}$. So, for $\prod \varphi_{m}: M \hookleftarrow \prod G_{m} \in \mathbf{H}, r \in K_{\mathbf{H}}(M)$ if and only if $r \in$ $1\left(\prod \varphi_{m}\right)^{-1}$. Now, for each $a \in A$, choose $\tilde{a} \in a\left(\prod \varphi_{m}\right)$. Then

$$
G=\langle\{\tilde{a} \mid a \in A\}\rangle \in \mathbf{H}
$$

is the desired group.

\section{Inverse Automata and Embedding Problems}

In this section, we obtain simple proofs of the results of [30] characterizing membership in the pseudovariety of inverse monoids $\mathbf{S l}(\mathrm{m} \mathbf{H}$. It turns out to be a geometric condition involving embeddings of inverse monoids in covers (or, from another viewpoint, extensions of partial one-to-one maps). Our approach is more elementary in that we avoid using profinite monoids. These techniques form the basis for our later work.

5.1. Inverse Automata. We first need the following well known result which can easily be proved from Lemma 7.1 below. If $M$ is any monoid, we use $\oplus \operatorname{Sch}(M)$ to denote the direct product of $S \operatorname{ch}(X)$ as $X$ ranges over the regular $\mathcal{R}$-classes of $M$ and $\oplus S \operatorname{ch}^{\rho}(M)$ to denote the direct product of $S \operatorname{ch}^{\rho}(Y)$ as $Y$ ranges over the regular $\mathcal{L}$-classes of $M$. 
Proposition 5.1. Let I be a finite inverse monoid. Then $I$ is a subdirect product of the monoids $S \operatorname{ch}(X)$ as $X$ ranges over the $\mathcal{R}$-classes of $I$; that is to say, the natural map from $I$ to $\oplus S \operatorname{ch}(I)$ is injective.

Thus, to deal with membership in the pseudovariety of inverse monoids $\mathbf{S l} m \mathbf{H}$, it suffices to handle the case of transition monoids of inverse automata. Our treatment is similar to that of [30], but we do not restrict to the special case of what they call reduced inverse automata. The principal difference is that we use the $\mathbf{H}$-kernel in the types of arguments where profinite monoids are used in [30].

Proposition 5.2. Let $G$ be a finite (or torsion) group, $I$ an inverse monoid, and $\varphi: I \leftrightarrow G$ a relational morphism of monoids. Then $\varphi$ is a relational morphism of inverse monoids; that is, $\varphi$ is a subinverse monoid of $I \times G$.

Proof. First note that $m^{-1}=\left(m^{-1} m\right)^{n} m^{-1}$ for all $n \geq 0$. Suppose that $(m, g) \in \varphi$. Let $m^{-1} \varphi h$. Then $\left(\left(m^{-1} m\right)^{n} m^{-1},(h g)^{n} h\right) \in \varphi$ for all $n \geq 0$. Let $k>0$ be such that $(h g)^{k}=1$. Then $(h g)^{k-1} h=g^{-1}$, and so $\left(m^{-1}, g^{-1}\right) \in \varphi$ as desired.

It now follows that the pseudovariety of inverse monoids $\mathbf{S l} m \mathbf{H}$ is nothing more than the collection of inverse monoids in the monoid pseudovariety $\mathbf{S l} m \mathbf{H}$. Also, if $I$ is a finite inverse monoid, it now follows $K_{\mathbf{H}}(I)$ is a subinverse monoid of $I$ and that if $n \in I$, then $n^{-1} K_{\mathbf{H}}(I) n \subseteq K_{\mathbf{H}}(I)$. The following proposition is easy to prove with our geometric methods. We recall for a finite cover $\Gamma$ that $M(\Gamma)=M(\mathcal{I}(\Gamma))$ and is a group.

Proposition 5.3. Let $\left(\mathcal{I}(\Gamma), q_{0}\right)$ be an inverse A-automaton. Then $M(\mathcal{I}(\Gamma)) \in$ $\mathbf{S l}\left[\mathbf{H}\right.$ if and only if there exists a finite cover $\Gamma^{\prime}$ with $\Gamma \subseteq \Gamma^{\prime}$ and $M\left(\Gamma^{\prime}\right) \in \mathbf{H}$.

Proof. Let $I=M(\mathcal{I}(\Gamma))$. Suppose first that such a $\Gamma^{\prime}$ exists and let $w \in \tilde{A}^{*}$ be such that $[w]_{M\left(\Gamma^{\prime}\right)}=1$. Then $w$ acts as the identity on $\Gamma^{\prime}$ and hence as a partial identity on $\Gamma$ whence $[w]_{I} \in E(I)$. But $E(I) \in \mathbf{S l}$; thus the canonical relational morphism of $I$ with $M\left(\Gamma^{\prime}\right)$ has Mal'cev kernel in $\mathbf{S l}$.

For the converse, choose, as in Proposition 4.3, a group $G \in \mathbf{H}$ which is $A$ generated and such that, for the canonical relational morphism $\mu: I \multimap G, 1 \mu^{-1}=$ $K_{\mathbf{H}}(I)=E(I)$. Let

$$
\operatorname{Stab}\left(q_{0}\right)=\left\{w \in \tilde{A}^{*} \mid q_{0} w=q_{0}\right\} .
$$

This is evidently a unary submonoid of $\tilde{A}^{*}$. Let

$$
H=\left\{g \in G \mid g=[w]_{G}, w \in \operatorname{Stab}\left(q_{0}\right)\right\},
$$

that is, the image of $\operatorname{Stab}\left(q_{0}\right)$ in $G$. Then $H$ is a subgroup of $G$. We let $\Gamma^{\prime}$ be the coset graph of $H: V\left(\Gamma^{\prime}\right)=G / H ; E^{+}\left(\Gamma^{\prime}\right)=G / H \times A ;(H g, a) \alpha=H g$; $(H g, a) \omega=H g[a]_{G}$; and the label of $(H g, a)$ is $a$. An edge $(H g, a)$ is drawn

$$
H g \stackrel{a}{\longrightarrow} H g[a]_{G} \text {. }
$$

Then $\left(\Gamma^{\prime}, H\right)$ is a complete inverse $A$-automaton, that is, a finite-sheeted cover of $B_{A}$. Let $H_{G}=\bigcap_{g \in G} g^{-1} H g$ be the core of $H$ in $G$. It is well known that $M\left(\Gamma^{\prime}\right)=G / H_{G}$.

We claim $\pi_{1}\left(\Gamma, q_{0}\right) \subseteq \pi_{1}\left(\Gamma^{\prime}, H\right)$. Indeed, if $w \in \tilde{A}^{*}$ reads a circuit at $q_{0}$, then $w \in \operatorname{Stab}\left(q_{0}\right)$, and so $[w]_{G} \in H$ whence the run of $w$ from $H$ is a circuit. Since $\Gamma^{\prime}$ is a cover of $B_{A}$, there is, by Theorem [3.3, a unique automaton morphism $\varphi:\left(\mathcal{I}(\Gamma), q_{0}\right) \rightarrow\left(\mathcal{I}\left(\Gamma^{\prime}\right), H\right)$ (note that a lifting of the labeling function of $\Gamma$ to $\Gamma^{\prime}$ 
with $q_{0}$ going to $H$ is the same thing as an automaton morphism). To show that the morphism is an embedding, it suffices to show that it is injective on vertices (since labelings are faithful). So let $q, q^{\prime} \in V(\Gamma)$ and suppose $q \varphi=q^{\prime} \varphi$. Let $q=q_{0} w_{1}$ and $q^{\prime}=q_{0} w_{2}$. Then

$$
H w_{1}=q \varphi=q^{\prime} \varphi=H w_{2}
$$

so $\left[w_{1} w_{2}^{-1}\right]_{G} \in H$. Hence there exists $v \in \operatorname{Stab}\left(q_{0}\right)$ such that $\left[v w_{1} w_{2}^{-1}\right]_{G}=1$. But then, by choice of $G,\left[v w_{1} w_{2}^{-1}\right]_{I}$ is an idempotent and hence acts as a partial identity; so $q_{0} v w_{1} w_{2}^{-1}=q_{0}$. But,

$$
q_{0} v w_{1} w_{2}^{-1}=q_{0} w_{1} w_{2}^{-1}=q w_{2}^{-1} .
$$

Thus

$$
q w_{2}^{-1}=q_{0}=q^{\prime} w_{2}^{-1}
$$

and so $q=q^{\prime}$. The result follows.

If an inverse automaton satisfies the equivalent conditions of the above theorem, we call it $\mathbf{H}$-extendible. Note that any inverse automaton is $\mathbf{G}$-extendible whence $\mathbf{S l} \rightarrow \mathbf{G}$ is the pseudovariety of all finite inverse monoids. We now show that there is a least automaton congruence on an inverse automaton $\left(\mathcal{I}(\Gamma), q_{0}\right)$ so that the quotient is $\mathbf{H}$-extendible. Our description is slightly different from, and more general than, the one given in 30 .

Theorem 5.4. Let $\left(\mathcal{I}(\Gamma), q_{0}\right)$ be an inverse A-automaton. We define, for $p, q \in$ $V(\Gamma), p \sim_{\mathbf{H}} q$ if there exists $m \in K_{\mathbf{H}}(M(\mathcal{I}(\Gamma)))$ such that $p m=q$. Then $\sim_{\mathbf{H}}$ is the least inverse automaton congruence on $\left(\mathcal{I}(\Gamma), q_{0}\right)$ with $\mathbf{H}$-extendible quotient. We call $\sim_{\mathbf{H}}$ the $\mathbf{H}$-extendible congruence.

Proof. Let $I=M(\mathcal{I}(\Gamma))$. Using that $K_{\mathbf{H}}(I)$ is a subinverse monoid, it is easy to see that $\sim_{\mathbf{H}}$ is an equivalence relation. Suppose $p \sim_{\mathbf{H}} p^{\prime}$ and $p a=q, p^{\prime} a=q^{\prime}$. Let $p^{\prime}=p m$ with $m \in K_{\mathbf{H}}(I)$. Then $q a^{-1} m a=q^{\prime}$ and $a^{-1} m a \in K_{\mathbf{H}}(I)$, so $q \sim_{\mathbf{H}} q^{\prime}$. The other verification that $\sim_{\mathbf{H}}$ is an inverse automaton congruence is dual. Let $\left(\mathcal{I}\left(\Gamma^{\prime}\right), v_{0}\right)=\left(\mathcal{I}(\Gamma), q_{0}\right) / \sim_{\mathbf{H}}$. Let $G \in \mathbf{H}$ be such that $m \in K_{\mathbf{H}}(I)$ if and only there is a word $w \in \tilde{A}^{*}$ with $[w]_{I}=m,[w]_{G}=1$. Consider the canonical relational morphism of $M\left(\mathcal{I}\left(\Gamma^{\prime}\right)\right)$ with $G$. Suppose $w \in \tilde{A}^{*}$ is such that $[w]_{G}=1$ and that $p, q \in V(\Gamma)$ are such that $[p]_{\mathbf{H}} w=[q]_{\mathbf{H}}$ where $[p]_{\mathbf{H}}$ and $[q]_{\mathbf{H}}$ denote the $\sim_{\mathbf{H}^{-}}$equivalence classes of $p$ and $q$, respectively. Then, by definition of $\sim_{\mathbf{H}}$ and choice of $G$, if $w=a_{1} \cdots a_{n}$ with $a_{j} \in \tilde{A}$, then there exist $u_{i} \in \tilde{A}^{*}(i=0, \ldots, n)$ such that $\left[u_{i}\right]_{G}=1$ and $p u_{0} a_{1} u_{1} \cdots u_{n-1} a_{n} u_{n}=q$. But then

$$
\left[u_{0} a_{1} u_{1} \cdots u_{n-1} a_{n} u_{n}\right]_{G}=[w]_{G}=1 .
$$

So, by choice of $G$ and the definition $\sim_{\mathbf{H}}$, we see that $p \sim_{\mathbf{H}} q$ whence $w$ acts as a partial identity on $V\left(\Gamma^{\prime}\right)$. Thus $M\left(\mathcal{I}\left(\Gamma^{\prime}\right)\right) \in \mathbf{S l} m \mathbf{H}$, and so $\left(\mathcal{I}\left(\Gamma^{\prime}\right), v_{0}\right)$ is H-extendible.

Suppose $(\mathcal{I}(\Delta), v)$ is another $\mathbf{H}$-extendible quotient of $\left(\mathcal{I}(\Gamma), q_{0}\right)$ and let $\varphi$ : $\left(\mathcal{I}(\Gamma), q_{0}\right) \rightarrow(\mathcal{I}(\Delta), v)$ be the projection morphism. Then if $p, q \in V(\Gamma)$ and $p \sim_{\mathbf{H}} q$, there exists $m \in K_{\mathbf{H}}(I)$ such that $p m=q$. Let $\Delta^{\prime}$ be a finite cover with $M\left(\Delta^{\prime}\right) \in \mathbf{H}$ and $\Delta \subseteq \Delta^{\prime}$. Then there exists $w \in \tilde{A}^{*}$ such that $[w]_{I}=m$ and $[w]_{M\left(\Delta^{\prime}\right)}=1$. Now, since $p w=q$ and $\Delta$ is quotient of $\Gamma, p \varphi w=q \varphi$. Hence, since $\Delta \subseteq \Delta^{\prime}, p \varphi=q \varphi$. The result follows. 
We have just shown that every inverse automaton $\mathcal{I}\left(\Gamma, q_{0}\right)$ has a maximal $\mathbf{H}$ extendible image which we shall denote by $\mathcal{I}_{\mathbf{H}}\left(\Gamma, q_{0}\right)$. We shall denote the underlying graph of this automaton $\mathcal{I}_{\mathbf{H}}(\Gamma)$ and the initial state by $\left[q_{0}\right]_{\mathbf{H}}$. These can be put together to give a functor from the category of inverse automata to the category of $\mathbf{H}$-extendible inverse automata, left adjoint to the forgetful functor (which is again an inclusion of a full subcategory).

Note that since one can effectively compute all the automaton congruences on a finite automaton, the decidability of $\mathbf{H}$-extendibility of an inverse automaton is equivalent to computability of the $\mathbf{H}$-extendible congruence, given an inverse automaton as input.

We have thus far reduced the membership problem to determining the least extendible congruence on an inverse automata. Before we can solve this problem, we need to recall the definition of the profinite topology on a group, due to Hall [23].

5.2. Profinite Topologies. Let $G$ be a group and $\mathbf{H}$ a pseudovariety of groups. Then the pro-H topology on $G$ is defined by taking as a basis of neighborhoods of 1 all normal subgroups $N$ of $G$ such that $G / N \in \mathbf{H}$. One then makes $G$ into a topological group in the standard way. This is the weakest topology on $G$ so that every homomorphism of $G$ to a group in $\mathbf{H}$ (endowed with the discrete topology) is continuous. We say that $G$ is residually in $\mathbf{H}$ if, for any $g \in G \backslash\{1\}$, there is a homomorphism $\varphi: G \rightarrow H \in \mathbf{H}$ with $g \varphi \neq 1$, or, equivalently, $\{1\}$ is a closed subgroup. In this case, the pro-H topology is Hausdorff and, in fact, metric. In general, the topology is given by the following ultrametric écart. For $g \in G$, define

$$
r(g)=\min (\{[G: N] \mid G / N \in \mathbf{H}, g \notin N\} \cup\{\infty\}) .
$$

Then the $\mathbf{H}$-pseudonorm is given by

$$
|g|_{\mathbf{H}}=2^{-r(g)}\left(\text { where } 2^{-\infty}=0\right) .
$$

One can verify that

$$
\left|g_{1} g_{2}\right|_{\mathbf{H}} \leq \max \left\{\left|g_{1}\right|_{\mathbf{H}},\left|g_{2}\right|_{\mathbf{H}}\right\}
$$

For $g_{1}, g_{2} \in G$, we define

$$
d_{\mathbf{H}}\left(g_{1}, g_{2}\right)=\left|g_{1} g_{2}^{-1}\right|_{\mathbf{H}} .
$$

It is easy to see that this is an ultrametric écart defining the pro- $\mathbf{H}$ topology which is a metric if and only if $G$ is residually in $\mathbf{H}$. If $\mathbf{H}$ is the pseudovariety $\mathbf{G}_{p}$ of $p$ groups for a prime $p$ and $G=\mathbb{Z}$, then this topology is just the usual $p$-adic topology on the integers and the above norm and metric are equivalent to the usual $p$-adic norm and metric. The following straightforward proposition is due to Hall [23]; see, for instance, 30].

Proposition 5.5. Let $G$ be a group, $H$ a subgroup, and $\mathbf{H}$ a pseudovariety of groups. Then the following are equivalent for the pro-H topology:

1. $H$ is open;

2. $H$ is closed of finite index;

3. $H$ is of finite index and $G / H_{G} \in \mathbf{H}$ where, as before, $H_{G}$ denotes the core of $H$ in $G$.

We use $c l_{\mathbf{H}}(X)$ to denote the closure of $X \subseteq G$ in the pro-H topology. For those not familiar with this topology, we offer the following "Rosetta stone:" if $g \in G$, then $g \in c l_{\mathbf{H}}(X)$ if and only if, for all $\varphi: G \rightarrow H \in \mathbf{H}, g \varphi \in X \varphi$. We note that since 
the pro-H topology is pseudometric, one has that $c l_{\mathbf{H}}(X \cup Y)=c l_{\mathbf{H}}(X) \cup c l_{\mathbf{H}}(Y)$. It is also not difficult to prove the following standard proposition which we leave as an exercise.

Proposition 5.6. Let $G$ be a group, $H$ a subgroup, and $\mathbf{H}$ a pseudovariety of groups. Then $\mathrm{cl}_{\mathbf{H}}(H)=\bigcap_{\text {open } K \supseteq H} K$.

From now on, we take $\mathbf{H}$ to be a fixed pseudovariety of groups. Let $A$ be a finite set and consider $F G(A)$ in the pro-H topology. If $F G(A)$ is not residually in $\mathbf{H}$, then $c l_{\mathbf{H}}(\{1\})$ is a normal, in fact, verbal subgroup of $F G(A)$ and $F G_{\mathbf{H}}(A)=$ $F G(A) / c l_{\mathbf{H}}(\{1\})$ is the relatively free group in the variety of groups generated by $\mathbf{H}$. Indeed, note that, by definition of the pro-H topology, $c l_{\mathbf{H}}(\{1\})$ consists precisely of those words $w \in F G(A)$ which map to 1 in every group in $\mathbf{H}$.

Let $M$ be a finite $A$-generated monoid (or inverse monoid). We define a relational morphism $\alpha_{\mathbf{H}}: M \nrightarrow F G(A)$ as follows. Give $M$ the discrete topology, $F G(A)$ the pro-H topology, and $M \times F G(A)$ the product topology. Let

$$
\alpha_{\mathbf{H}}=c l_{\mathbf{H}}\left(\left\langle\left([a]_{M},[a]_{F G(A)}\right)\right\rangle\right)
$$

(where if $M$ is an inverse monoid, we take the subinverse monoid generated by the above pairs). We note that if

$$
L_{m}=\left\{u \in A^{*} \mid[u]_{M}=m\right\},
$$

then $m \alpha_{\mathbf{H}}=\operatorname{cl}_{\mathbf{H}}\left(L_{m}\right)$ for $M$ a monoid. For $M$ an inverse monoid,

$$
m \alpha_{\mathbf{H}}=c l_{\mathbf{H}}\left(\left\{[w]_{F G(A)} \mid w \in \tilde{A}^{*},[w]_{M}=m\right\}\right) .
$$

Proposition 5.7. Let $M$ be a finite A-generated monoid (or inverse monoid). The $m \in K_{\mathbf{H}}(M)$ if and only if $m \in 1 \alpha_{\mathbf{H}}^{-1}$.

Proof. We just handle the monoid case, the inverse monoid case being identical. Let $G \in \mathbf{H}$ be an $A$-generated group such that, for the canonical relational morphism $\mu: M \multimap G, m \in K_{\mathbf{H}}(M)$ if and only if $m \in 1 \mu^{-1}$. Let $\varphi: F G(A) \rightarrow G$ be the canonical surmorphism and $N=\operatorname{ker} \varphi$. Suppose now that $1 \in \operatorname{cl}_{\mathbf{H}}\left(L_{m}\right)$. Then, since $N$ is an open subgroup, $N \cap L_{m}$ is non-empty, so there exists $w \in A^{*}$ such that $[w]_{M}=m$ and $[w]_{G}=1$. Thus $m \in K_{\mathbf{H}}(M)$.

Conversely, if $m \in K_{\mathbf{H}}(M)$, then, for any open normal subgroup $N \in F G(A)$, we see that $L_{m} \cap N$ is non-empty by considering the canonical relational morphism of $M$ with $F G(A) / N \in \mathbf{H}$. So $1 \in c l_{\mathbf{H}}\left(L_{m}\right)$.

Corollary 5.8. Let $\left(\mathcal{I}(\Gamma), q_{0}\right)$ be an inverse A-automaton and $\varphi: \tilde{A}^{*} \rightarrow F G(A)$ the natural projection. Let $L_{p, q}=\left\{w \in \tilde{A}^{*} \mid p w=q\right\}$. Then $p \sim_{\mathbf{H}} q$ if and only if $1 \in \operatorname{cl}_{\mathbf{H}}\left(L_{p, q} \varphi\right)$ in the pro-H topology.

Proof. Let $I=M(\mathcal{I}(\Gamma))$. If there exists $m \in K_{\mathbf{H}}(I)$ such that $p m=q$, then, by Proposition 5.7, $1 \in c l_{\mathbf{H}}\left(L_{p, q} \varphi\right)$. For the converse, let $G \in \mathbf{H}$ be $A$-generated such that $m \in K_{\mathbf{H}}(I)$ if and only if, for the canonical relational morphism $\mu: I \multimap G$, $m \in 1 \mu^{-1}$. Then, since $1 \in \operatorname{cl}_{\mathbf{H}}\left(L_{p, q} \varphi\right)$, it follows that there exists $w \in L_{p . q}$ such that $[w]_{G}=1$. Then $m=[w]_{I} \in K_{\mathbf{H}}(I)$ and $p m=q$, so $p \sim_{\mathbf{H}} q$.

We now calculate $c l_{\mathbf{H}}\left(L_{p, q}\right)$.

Lemma 5.9. Let $\left(\mathcal{I}(\Gamma), q_{0}\right)$ be an inverse $A$-automaton, $p, q \in V(\Gamma), w_{p}$ a reduced word reading from $q_{0}$ to $p$, and $w_{q}$ a reduced word reading from $q_{0}$ to $q$. Let $L_{p, q}=$ 
$\left\{w \in \tilde{A}^{*} \mid p w=q\right\}$ and $\varphi: \tilde{A}^{*} \rightarrow F G(A)$ the canonical projection. Then $L_{p, q} \varphi=$ $w_{p}^{-1} \pi_{1}\left(\Gamma, q_{0}\right) w_{q}$.

Proof. Clearly, $w_{p}^{-1} \pi_{1}\left(\Gamma, q_{0}\right) w_{q} \subseteq L_{p, q} \varphi$. Conversely, if $w \in L_{p, q}$, then $w_{p} w w_{q}^{-1}$ is a loop at $q_{0}$. It follows that $[w]_{F G(A)} \in w_{p}^{-1} \pi_{1}\left(\Gamma, q_{0}\right) w_{q}$.

Since translation is a homeomorphism, we get the following.

Lemma 5.10. Let $G$ be a group in the pro-H topology, $X \subseteq G$, and $g \in G$. Then $c l_{\mathbf{H}}(X g)=c l_{\mathbf{H}}(X) g$ and $c l_{\mathbf{H}}(g X)=g\left(c l_{\mathbf{H}}(X)\right)$.

Thus from Lemma 5.9 we obtain the following.

Corollary 5.11. Let $\left(\mathcal{I}(\Gamma), q_{0}\right)$ be an inverse A-automaton, $p, q \in V(\Gamma)$, w $w_{p}$ a reduced word reading from $q_{0}$ to $p$, and $w_{q}$ a reduced word reading from $q_{0}$ to $q$. Then

$$
c l_{\mathbf{H}}\left(L_{p, q} \varphi\right)=w_{p}^{-1} c l_{\mathbf{H}}\left(\pi_{1}\left(\Gamma, q_{0}\right)\right) w_{q} .
$$

Now we can obtain a characterization of the least $\mathbf{H}$-extendible congruence for an inverse automaton. This result was obtained in the special case of a reduced automaton in [30].

Theorem 5.12. Let $\left(\mathcal{I}(\Gamma), q_{0}\right)$ be an inverse A-automaton, $p, q \in V(\Gamma), w_{p}$ a reduced word reading from $q_{0}$ to $p$, and $w_{q}$ a reduced word reading from $q_{0}$ to $q$. Then $p \sim_{\mathbf{H}} q$ if and only if $w_{p} w_{q}^{-1} \in \operatorname{cl}_{\mathbf{H}}\left(\pi_{1}\left(\Gamma, q_{0}\right)\right)$.

Proof. By Corollary [5.8, $p \sim_{\mathbf{H}} q$ if and only if $1 \in c l_{\mathbf{H}}\left(L_{p, q} \varphi\right)$. So, by the above corollary, $1 \in c l_{\mathbf{H}}\left(L_{p, q} \varphi\right)$ if and only if $w_{p} w_{q}^{-1} \in c l_{\mathbf{H}}\left(\pi_{1}\left(\Gamma, q_{0}\right)\right)$.

We are thus led to the following result of [30].

Theorem 5.13. Let $\mathbf{H}$ be a pseudovariety of groups. Suppose that there is an algorithm, for each finite set $A$, to determine, given as input a finite set $Y$ of reduced words over $A$, membership in $c l_{\mathbf{H}}(\langle Y\rangle)$ in the pro-H topology. Then the membership problem for the pseudovariety of inverse monoids $\mathbf{S l} m \mathbf{H}$ is decidable in only polynomially worse time.

Proof. If $I$ is a finite $A$-generated inverse monoid, then it is well known that the automaton $\left(\operatorname{Sch}_{A}(X), e_{X}\right)$ for each $\mathcal{R}$-class $X$ of $I$ (with corresponding idempotent $\left.e_{X}\right)$ can be constructed in polynomial time. By Proposition 5.1, $I \in \mathbf{S l} m \mathbf{H}$ if and only if $M\left(\mathcal{I}\left(S c h_{A}(X)\right)\right) \in \mathbf{S l} m \mathbf{H}$ for every $\mathcal{R}$-class $X$. But this occurs if and only if each such $\mathcal{I}\left(\operatorname{Sch}_{A}(X)\right)$ is $\mathbf{H}$-extendible. By Theorem 5.12 this can be checked in only polynomial worse time than the membership problem for $c l_{\mathbf{H}}\left(\pi_{1}\left(\operatorname{Sch}_{A}(X), e_{X}\right)\right)$. But we can find a finite basis for $\pi_{1}\left(\operatorname{Sch}_{A}(X), e_{X}\right)$ in polynomial time so the result follows.

Now one can ask: What does $\mathbf{H}$-extendibility mean in terms of the fundamental group of an inverse automaton? The answer is simple [30].

Proposition 5.14. Let $\mathbf{H}$ be a pseudovariety of groups and suppose that $\left(\mathcal{I}(\Gamma), v_{0}\right)$ is an $\mathbf{H}$-extendible inverse A-automaton. Then $\pi_{1}\left(\Gamma, v_{0}\right)$ is a free factor in an $\mathbf{H}$ open subgroup $K \subseteq F G(A)$.

Proof. Let $\Delta$ be a finite cover with $\Gamma \subseteq \Delta$ and $M(\Delta)$ in $\mathbf{H}$. Then it is easy to see that $\Delta$ is the coset graph of $K=\pi_{1}\left(\Delta, v_{0}\right)$, so $M(\Delta)=F G(A) / K_{F G(A)}$. Hence $K$ is open by Proposition [5.5. Now any maximal subtree of $\Gamma$ can be extended to one of $\Delta$, so there is a basis for $K$ containing a basis for $\pi_{1}\left(\Gamma, v_{0}\right)$. 
The following result can be found in [30, 38]. A non-trivial pseudovariety $\mathbf{H}$ of groups is said to be extension-closed if $\mathbf{H} * \mathbf{H}=\mathbf{H}$, or, equivalently, $1 \rightarrow N \rightarrow$ $G \rightarrow H \rightarrow 1$ an exact sequence of groups with $N, H \in \mathbf{H}$, implies that $G \in \mathbf{H}$. For instance, the pseudovarieties $\mathbf{G}, \mathbf{G}_{p}$, and $\mathbf{G}_{\text {sol }}$ are extension-closed, while $\mathbf{G}_{n i l}$ is not.

Proposition 5.15. Let $\mathbf{H}$ be an extension-closed pseudovariety. Suppose $H$ is a subgroup of $F G(A)$ which is a free factor in an open subgroup $K$, then $H$ is finitely generated and closed in the pro-H topology.

It should be pointed out that, for any pseudovariety $\mathbf{H}$ of groups, if $H \subseteq F G(A)$ is a finitely generated closed subgroup, then it is a free factor in an open subgroup. To show this, we construct, following the algorithm of Stallings [40], a finite inverse automaton with $H$ as its fundamental group. Then, following [30], we show that this automaton is $\mathbf{H}$-extendible; the claim will then follow. Let $Y$ be a finite set of reduced words such that $H=\langle Y\rangle$. Form an $A$-graph $\Delta$ as follows. First, for each element $w$ of $Y$, take a circle subdivided into $|w|$ parts reading the word $w$ from some chosen base point; then identify all these base points. The resulting graph $\Delta$ is an $A$-graph which is a wedge of subdivided circles each reading an element of $Y$. Let $w_{0}$ be the base point. One then has that $\pi_{1}\left(\Delta, w_{0}\right)$ is a free group on $|Y|$ generators and if $\ell:\left(\Delta, w_{0}\right) \rightarrow\left(B_{A}, 1\right)$ is the labeling, then $\pi_{1}\left(\Delta, w_{0}\right) \ell=\langle Y\rangle$. Let $\Gamma=\mathcal{I}(\Delta)$ and $v_{0}$ be the equivalence class of $w_{0}$. Since $\Gamma$ is obtained by factoring $\ell$ through a sequence of folds, and folds are surjective on fundamental groups, it follows that $\pi_{1}\left(\Gamma, v_{0}\right)=\pi_{1}\left(\Delta, w_{0}\right) \ell=\langle Y\rangle$. Now suppose $p, q \in V(\Gamma)$ with $p \sim_{\mathbf{H}} q$. Let $w_{p}$ and $w_{q}$ be reduced words reading from $v_{0}$ to $p$ and $q$ respectively. Then, since $H=c l_{\mathbf{H}}(H)$ by hypothesis, $w_{p} w_{q}^{-1} \in c l_{\mathbf{H}}(H)$ implies $w_{p} w_{q}^{-1} \in H$, and hence $p=q$. We thus have the following proposition of [30].

Proposition 5.16. Let $\mathbf{H}$ be a pseudovariety of groups and $H \subseteq F G(A)$ be a finitely generated subgroup, closed in the pro-H topology. Then $H$ is a free factor of an open subgroup $K$.

Since any inverse automaton is $\mathbf{G}$-extendible, we recover some old theorems of Hall [22, 23, 40.

Theorem 5.17. Let $H \subseteq F G(A)$ be finitely generated. Then $H$ is a free factor in a $\mathbf{G - o p e n ~ s u b g r o u p ~ a n d ~ h e n c e ~ i s ~ c l o s e d ~ i n ~ t h e ~ p r o - G ~ t o p o l o g y . ~}$

A related result of Ribes and Zalesskiu, see [30. 38], is the following.

Proposition 5.18. Let $\mathbf{H}$ be an extension-closed pseudovariety of groups and $H \subseteq$ $F G(A)$ be a finitely generated subgroup. Then $\operatorname{rank}\left(\operatorname{cl}_{\mathbf{H}}(H)\right) \leq \operatorname{rank}(H)$. In particular, $\operatorname{cl}_{\mathbf{H}}(H)$ is finitely generated.

The following is a deep result of Ribes and Zalesskiu [37 38].

Theorem 5.19. Let $\mathbf{H}$ be an extension-closed pseudovariety of groups and let $H_{1}, \ldots, H_{n}$ be finitely generated, closed subgroups of $F G(A)$ in the pro-H topology. Then $H_{1} \cdots H_{n}$ is closed.

We end this subsection by remarking that in [30] algorithms are given to compute membership in the $\mathbf{H}$-closure of a finitely generated subgroup of a free group for the pseudovarieties $\mathbf{G}_{p}$ of $p$-groups and $\mathbf{G}_{n i l}$ of nilpotent groups, while in [42] the author solves this problem for pseudovarieties with a decidable membership problem, consisting entirely of abelian groups. 
5.3. Some Connections to Decidability Results from Group Theory. We now present an argument for (a generalization of) an unpublished result of Margolis [26]. The proof is based on the arguments used by Rhodes [35] and the author (with Rhodes) [36] for a monoid analog, but is of a geometric nature, using arguments involving covers.

Let $w \in \tilde{A}^{*}$ be a reduced word. A group $G$ is said to satisfy the group identity $(w=1)$, written $G \models(w=1)$, if, for every function $\varphi: A \rightarrow G, w \tilde{\varphi}=1$ where $\tilde{\varphi}$ is the unique extension of $\varphi$ to $F G(A)$. More generally, if $E$ is a set of group identities, then $G \models E(\operatorname{read} G$ satisfies $E)$ if, for each identity $(w=1) \in E, G \models(w=1)$. If $\mathbf{H}$ is a pseudovariety of groups and $E$ a set of group identities, we say $\mathbf{H}=E$ if, for every $G \in \mathbf{H}, G \models E$. Let $w \in \tilde{A}^{*}$ be a reduced word. Then an easy exercise shows that $\mathbf{H} \models(w=1)$ if and only if $w \in c_{\mathbf{H}}(\{1\})$. Let $E_{\mathbf{H}}$ be the set of all group identities of the form $(w=1)$ satisfied by $\mathbf{H}$ with $w$ a reduced word over a fixed, countable, recursively enumerable set; we call $E_{\mathbf{H}}$ the equational theory of $\mathbf{H}$. We say $\mathbf{H}$ has decidable equational theory if $E_{\mathbf{H}}$ is recursive. Hence if, for each finite set $A$, there is an algorithm to compute membership in $c l_{\mathbf{H}}(\langle Y\rangle) \subseteq F G(A)$, given a finite set $Y$ of reduced words over $\tilde{A}$, then the equational theory of $\mathbf{H}$ is decidable. Our next goal is to show that if the pseudovariety of inverse monoids $\mathbf{S l} m \mathbf{H}$ is decidable, then so is the equational theory of $\mathbf{H}$.

First, we show a connection between embeddings of inverse automata in covers and the word problem for groups. We start by discussing the word problem for $F I M(A)$. Let $w \in \tilde{A}^{*}$. Then the Munn tree of $w, \Gamma(w)$, is the subgraph of $\Gamma_{A}(F G(A))$ used in the run of $w$ from 1 . The result is then that $[w]_{F I M(A)}=$ $\left[w^{\prime}\right]_{F I M(A)}$ if and only if $[w]_{F G(A)}=\left[w^{\prime}\right]_{F G(A)}$ and $\Gamma(w)=\Gamma\left(w^{\prime}\right)$. Given a reduced word $w \in \tilde{A}^{*}$, we then consider the inverse $A$-automaton $(\mathcal{I}(\Gamma(w)), 1)$. Define Test $(w)$ to be the set of all inverse automaton quotients of $(\mathcal{I}(\Gamma(w)), 1)$ such that the states of 1 and $w$ do not get identified. Then we have the following simple lemma.

Lemma 5.20. Let $G$ be an A-generated (perhaps infinite) group and $w \in \tilde{A}^{*} a$ reduced word. Then $[w]_{G}=1$ if and only if no element of Test $(w)$ can be embedded in $\Gamma_{A}(G)$.

Proof. We first note that $\Gamma_{A}(G)$ is a regular covering of $B_{A}$ : a word $v \in \tilde{A}$ reads a loop at one vertex of $G$ if and only if it reads a loop at every vertex. So suppose $\left(\mathcal{I}(\Gamma), v_{0}\right) \in \operatorname{Test}(w)$ is such that $\Gamma \subseteq \Gamma_{A}(G)$. Then, since $v_{0} w \neq v_{0}$ by definition of Test $(w), w$ does not read a loop at any vertex of $\Gamma_{A}(G)$, so $[w]_{G}=[1]_{G} w \neq[1]_{G}$.

Suppose, on the other hand, that $[w]_{G} \neq 1$. Since $\Gamma(w)$ is a tree, $\pi_{1}(\Gamma(w), 1)=$ 1. Then, since $\Gamma_{A}(G)$ is a cover, we obtain, by Theorem 3.3 a unique inverse automaton morphism $\varphi:(\mathcal{I}(\Gamma(w)), 1) \rightarrow\left(\Gamma_{A}(G),[1]_{G}\right)$. Let $\sim_{\varphi}$ be the associated inverse automaton congruence on $(\mathcal{I}(\Gamma(w)), 1)$. Then, since $[w]_{G} \neq[1]_{G}, 1 \varphi \neq w \varphi$. Hence $(\mathcal{I}(\Gamma(w)), 1) / \sim_{\varphi} \in$ Test $(w)$ which embeds in $\Gamma_{A}(G)$.

Theorem 5.21. It is undecidable whether, given as input a finite inverse A-automaton $\left(\mathcal{I}(\Gamma), q_{0}\right)$ over some finite set $A$ and a finite presentation of a group $G$ generated by $A$, whether $\left(\mathcal{I}(\Gamma), q_{0}\right)$ can be embedded in the Cayley graph $\Gamma_{A}(G)$.

Proof. The above lemma shows that the decidability of the problem in question would imply the decidability of the word problem which is well known to be undecidable. 
Now we obtain the following unpublished result of Margolis (actually, my understanding is that he proved the result for equational pseudovarieties).

Theorem 5.22. Let $\mathbf{H}$ be a pseudovariety of groups. Then if the pseudovariety of inverse monoids $\mathbf{S l} \rightarrow \mathbf{H}$ has decidable membership problem, $\mathbf{H}$ has decidable equational theory.

Proof. Let $w \in \tilde{A}^{*}$ be a reduced word. We claim $\mathbf{H}=(w=1)$ if and only if no element of Test $(w)$ has transition monoid in $\mathbf{S l} m \mathbf{H}$. The result follows from this. Suppose first that some element $\left(\mathcal{I}(\Gamma), v_{0}\right)$ of Test $(w)$ has $M(\mathcal{I}(\Gamma)) \in \mathbf{S l}(m \mathbf{H}$ and that $\mathbf{H} \models(w=1)$. Then $\left(\mathcal{I}(\Gamma), v_{0}\right)$ is $\mathbf{H}$-extendible and so there is a cover $\Delta$ with $M(\Delta) \in \mathbf{H}$ and $\Gamma \subseteq \Delta$. But then $[w]_{M(\Delta)}=1$ whence $w$ acts a partial identity on $V(\Gamma)$. But since $1 w=w$ in $\Gamma(w)$, it follows $v_{0} w$ is the equivalence class in $V(\Gamma)$ of $w$, and so 1 and $w$ are equivalent in $V(\Gamma)$, contradicting that $\left(\mathcal{I}(\Gamma), v_{0}\right) \in \operatorname{Test}(w)$.

Suppose now that $\mathbf{H}$ does not satisfy $(w=1)$. Then there is a group $G \in \mathbf{H}$ for which $[w]_{G} \neq 1$. Hence, by Lemma $\left[5.20\right.$ some element $\left(\Gamma, v_{0}\right) \in \operatorname{Test}(w)$ can be embedded in $\Gamma_{A}(G)$. So $M(\mathcal{I}(\Gamma)) \in \mathbf{H}$.

As far as the author knows, it is open as to whether there exists a pseudovariety $\mathbf{H}$ of groups with decidable membership problem but whose equational theory is undecidable. In fact, we believe the following special case is still open. Let $A$ be a finite set and $E$ a finite set of group identities of the form $(w=1)$ with $w$ a reduced word in $\tilde{A}^{*}$. Then the set of all finite groups satisfying $E$ forms a pseudovariety $\llbracket E \rrbracket$ which evidently has decidable membership. The identity problem for groups asks whether, for every finite set of group identities $E$, there is an algorithm to determine, given a reduced word $w$ in variables $A$ as input, whether $G \models E$ implies $G \models(w=1)$ for any group $G$. This is known to be undecidable; see [1] for a history and references. The finite identity problem for groups asks the same question, but the groups $G$ are restricted to be finite. Equivalently, this question asks whether the equational theory of $\llbracket E \rrbracket$ is decidable for every finite set $E$ of identities. We believe that this question remains open, although the analogous question for semigroups is undecidable [1]. The above theorem shows that if there is a finite set of group identities $E$ for which one cannot decide if $(w=1)$ is a consequence in finite groups for all reduced words $w$, then $\mathbf{S l}(m \llbracket \llbracket E \rrbracket$ has an undecidable membership problem as a pseudovariety of inverse monoids (and hence also as a pseudovariety of monoids). We shall see later that several other algorithmic problems in which we are interested are equivalent to the membership problem for $\mathbf{S l} m \mathbf{H}$. We do note, however, that the undecidability of the identity problem for groups implies, by the above arguments, that there exists a variety of groups $\mathbf{H}$ for which one can decide membership for finite groups, but for which one cannot decide membership for finite inverse monoids in the inverse monoid variety $\mathbf{S l} m \mathbf{H}$ (defined analogously to the pseudovariety).

\section{Geometric Automata Theory}

In this section, we apply our ideas to automata in general. The key idea is to study the strongly connected components of an automaton.

6.1. Strongly Connected Automata. We have so far shown that, for any pseudovariety $\mathbf{H}$ of groups, there is a functor $\left(\Gamma, v_{0}\right) \mapsto \mathcal{I}_{\mathbf{H}}\left(\Gamma, v_{0}\right)$ from the category of non-deterministic $A$-automata to the category of $\mathbf{H}$-extendible inverse $A$-automata 
obtained by first folding edges to arrive at $\mathcal{I}\left(\Gamma, v_{0}\right)$ and then imposing $\sim_{\mathbf{H}}$. We wish to study this functor algebraically when restricted to the subcategory of strongly connected automata. Note that an automaton quotient of a strongly connected automaton is strongly connected, so these functors take strongly connected automata to strongly connected automata.

We first observe that any finite monoid can be made into a unary monoid in a natural way. If $M$ is a finite monoid and $m \in M$, then there is a unique idempotent $e$ which is a positive power of $m$. We denote this idempotent by $m^{\omega}$. There is also a unique element $r \in\langle m\rangle$ which is regular and such that $m r=m^{\omega}$. We call this element $m^{\omega-1}$ (note: $m^{\omega-1} m=m^{\omega}=m m^{\omega-1}$ ). One can verify that if $n \geq|M|$, then $m^{\omega}=m^{n !}$ and $m^{\omega-1}=m^{2 n !-1}$. Also, one can verify that if $\varphi: M \rightarrow N$ is a homomorphism, then $m^{\omega-1} \varphi=(m \varphi)^{\omega-1}$. So if we treat ()$^{\omega-1}$ as a unary operation, all homomorphisms of finite monoids are unary monoid homomorphisms. It then follows that any relational morphism $\mu: M \hookrightarrow N$ of finite monoids is automatically a relational morphism of unary monoids; that is, if $(m, n) \in \mu$, then $\left(m^{\omega-1}, n^{\omega-1}\right)=(m, n)^{\omega-1} \in \mu$. Let $\mathbf{M}^{\omega-1}$ denote the variety of unary monoids generated by finite monoids with this unary operation. Then, for each set $A$, we use $F^{\omega-1}(A)$ for the free unary monoid in this variety. Note that if $G$ is a group, then $G \in \mathbf{M}^{\omega-1}$ and ()$^{\omega-1}$ is just the usual inverse. This follows from noting that the free group on any generating set is residually finite and that the ()$^{\omega-1}$ operator is just the ordinary inverse for a finite group. Hence there is a natural projection $\rho: F^{\omega-1}(A) \rightarrow F G(A)$. For any element $m$ of a monoid in $\mathbf{M}^{\omega-1}$, we define $m^{\omega}=m^{\omega-1} m$. Then the identities $\left(x^{\omega}\right)^{n}=x^{\omega}(n \geq 1),\left(x^{\omega}\right)^{\omega}$, and $x x^{\omega-1}=x^{\omega}$ hold in $\mathbf{M}^{\omega-1}$.

This above discussion concerns a special case of the notion of an implicit operation; see [2, 7] for the relationship between implicit operations, profinite monoids, and pseudovarieties. Since we shall not make use of any other implicit operations in this paper, we avoid developing the notion. We do note, however, that many of the pseudovarieties which we have been considering are definable by a set of unary monoid identities, meaning that a finite monoid, viewed as a unary monoid via the ()$^{\omega-1}$-operation, is in the pseudovariety if and only if it satisfies the unary monoid identities in this set. For instance, $\mathbf{G}=\llbracket x^{\omega}=1 \rrbracket, \mathbf{R}=\llbracket(x y)^{\omega} x=(x y)^{\omega} \rrbracket$, $\mathbf{L}=\llbracket x(y x)^{\omega}=(y x)^{\omega} \rrbracket$, and $\mathbf{J}=\llbracket(x y)^{\omega} x=(x y)^{\omega}, x(y x)^{\omega}=(y x)^{\omega} \rrbracket$. If $\left(\Gamma, v_{0}\right)$ is an $A$-automaton and $p$ a state, then, for any term $\underline{w} \in F^{\omega-1}(A)$, we define $p \underline{w}=p[\underline{w}]_{M(\Gamma)}$.

We now study the relationship between runs of words from $\tilde{A}^{*}$ in $\mathcal{I}(\Gamma)$ and terms of $F^{\omega-1}(A)$. We say an element $\underline{w} \in F^{\omega-1}(A)$ has height at most one if it can be represented by a term with no nested occurrences of ()$^{\omega-1}$. For instance, $(b a)^{\omega-1} b$ has height at most one.

Lemma 6.1. Let $\left(\Gamma, v_{0}\right)$ be a strongly connected A-automaton, $a \in A$, and suppose $p a=q$. Then there exists $t \in A^{*}$ such that $q(t a)^{\omega-1} t=p$.

Proof. Since $\Gamma$ is strongly connected, there exists $t \in A^{*}$ such that $q t=p$. It is then easy to see that $q(t a)^{\omega-1} t=p$.

This lemma says that traversing an edge labeled by $a$ in the reverse direction can be accomplished by a term of $F^{\omega-1}(A)$ which maps to $a^{-1}$ in $F G(A)$. Hence, given any run in $\Gamma$ of a word in $\tilde{A}^{*}$, we can, by replacing each reverse transition 
with an appropriate $(\omega-1)$-term, obtain an $(\omega-1)$-term of height at most one with the same effect.

Lemma 6.2. Let $\left(\Gamma, v_{0}\right)$ be a strongly connected A-automaton, $w \in \tilde{A}^{*}$, and suppose that there is a run of $w$ from $p$ to $q$. Then there exists $\underline{w} \in F^{\omega-1}(A)$ of height at most one such that $p \underline{w}=q$ and $[\underline{w}]_{F G(A)}=[w]_{F G(A)}$.

Proof. We induct on $|w|$. If $|w|=0$, then $\underline{w}=1$ clearly works. Suppose $|w|=n$. Then if $w=v \tilde{a}, \tilde{a} \in \tilde{A}$, we see by induction that there exists $\underline{v} \in F^{\omega-1}(A)$ of height at most one with $p \underline{v}=q^{\prime}$, the vertex reached on reading $v$ in the run of $w$ from $p$, and $[\underline{v}]_{F G(A)}=[v]_{F G(A)}$. If $\tilde{a}=a \in A$, then $\underline{w}=\underline{v} a$ is as desired. If $\tilde{a}=a^{-1}$, with $a \in A$, then, since $q a=q^{\prime}$, there exists, by Lemma 6.1 $t \in A^{*}$ such that $q^{\prime}(t a)^{\omega-1} t=q$. Then the term $\underline{w}=\underline{v}(t a)^{\omega-1} t$ is as desired.

Proposition 6.3. Let $\left(\Gamma, v_{0}\right)$ be a strongly connected A-automaton and $w \in \tilde{A}^{*}$. We use $[p]$ for the equivalence class of a vertex $p$ in $\mathcal{I}\left(\Gamma, v_{0}\right)$. Suppose $p, q \in V(\Gamma)$ are such that $[p] w=[q]$. Then there exists $\underline{w} \in F^{\omega-1}(A)$ of height at most one such that $p \underline{w}=q$ and $[\underline{w}]_{F G(A)}=[w]_{F G(A)}$.

Proof. Suppose $w=a_{1} \cdots a_{n}$ with the $a_{i} \in \tilde{A}$. Then, by definition of $\mathcal{I}(\Gamma)$, there exist $u_{i} \in \tilde{A}^{*}(i=0, \ldots, n)$ such that $u_{0} a_{1} u_{1} a_{2} \cdots u_{n-1} a_{n} u_{n}$ has a run from $p$ to $q$ in $\Gamma$ and $\left[u_{i}\right]_{F G(A)}=1$ for all $i$. Thus, by the above lemma, there is a term $\underline{w} \in F^{\omega-1}(A)$ of height at most one such that $p \underline{w}=q$ and

$$
[\underline{w}]_{F G(A)}=\left[u_{0} a_{1} \cdots a_{n} u_{n}\right]_{F G(A)}=[w]_{F G(A)} .
$$

We remark that if $M$ is a finite $A$-generated monoid, $m \in M$, and $\underline{w} \in F^{\omega-1}(A)$ such that $[\underline{w}]_{M}=m$ and $[\underline{w}]_{F G(A)}=1$, then $m \in K_{\mathbf{G}}(M)$ (consider a relational morphism as per Proposition 4.3). The Rhodes type II conjecture is equivalent to the converse; see [5]. We then have the following corollary (compare to [48]).

Corollary 6.4. Let $\left(\Gamma, v_{0}\right)$ be a strongly connected A-automaton, then $[p]=[q]$ in $\left(\mathcal{I}(\Gamma), v_{0}\right)$ if and only if there exists $m \in K_{\mathbf{G}}(M(\Gamma))$ such that $p m=q$.

Proof. In light of the above remark, Proposition 6.3 shows that the condition is necessary. For sufficiency, consider any embedding of $\mathcal{I}(\Gamma)$ in a finite cover $\Delta$. Then, since $m \in K_{\mathbf{G}}$, there exists $u \in A^{*}$ such that $[u]_{M}=m$ and $[u]_{M(\Delta)}=1$. But $[p] u=[q]$ whence $[p]=[q]$.

Our next goal is to characterize in a similar manner the congruence on a strongly connected $A$-automaton $\left(\Gamma, v_{0}\right)$ associated to taking $\mathcal{I}_{\mathbf{H}}\left(\Gamma, v_{0}\right)$. If $\mathbf{V}$ is a pseudovariety of monoids, we denote by $\mathbf{E V}$ the pseudovariety consisting of finite monoids $M$ such that $\langle E(M)\rangle \in \mathbf{V}$.

Proposition 6.5. Let $\left(\Gamma, v_{0}\right)$ be a strongly connected A-automaton. Then the following are equivalent:

1. The A-graph $\Gamma$ is inverse;

2. $M(\Gamma) \in \mathbf{S l}(m) \mathbf{G}$;

3. $E(M(\Gamma)) \in \mathbf{S l}$;

4. $M(\Gamma) \in \mathbf{E R}$. 
Proof. Let $M=M(\Gamma)$. If $\Gamma$ is an inverse $A$-graph, then we can embed it in a finite cover $\Delta$. Hence, by considering the canonical relational morphism with $M(\Delta)$, we see that $M \in \mathbf{S l} m \mathbf{G}$. Since $E(M) \subseteq K_{\mathbf{G}}(M)$, if $M \in \mathbf{S l} m \mathbf{G}$, then $E(M) \in \mathbf{S l}$. Since $\mathbf{S l} \subseteq \mathbf{R}$, we are left with showing that if $M \in \mathbf{E R}$, then $\Gamma$ is an inverse $A$-graph. Since $\Gamma$ is deterministic, we need to show that if $p a=q$ and $p^{\prime} a=q$, then $p=p^{\prime}$. By Lemma 6.1, there exist $t, t^{\prime} \in A^{*}$ such that $q(t a)^{\omega-1} t=p$ and $q\left(t^{\prime} a\right)^{\omega-1} t^{\prime}=p^{\prime}$. Now

$$
p\left(a t^{\prime}\right)^{\omega}=p\left(a t^{\prime}\right)\left(a t^{\prime}\right)^{\omega-1}=q\left(t^{\prime} a\right)^{\omega-1} t^{\prime}=p^{\prime} .
$$

Similarly, $p^{\prime}(a t)^{\omega}=p$. Let $e_{1}=\left(a t^{\prime}\right)^{\omega}$ and $e_{2}=(a t)^{\omega}$. Then $p\left(e_{1} e_{2}\right)^{\omega} e_{1}=p^{\prime}$ and $p\left(e_{1} e_{2}\right)^{\omega}=p$. Since $\left(e_{1} e_{2}\right)^{\omega} \mathcal{R}\left(e_{1} e_{2}\right)^{\omega} e_{1}$ in $\langle E(M)\rangle$, they are equal and hence $p=p^{\prime}$. The result follows.

To describe $\mathcal{I}_{\mathbf{H}}\left(\Gamma, v_{0}\right)$ in terms of $M(\Gamma)$, we must first understand the relationship between $K_{\mathbf{H}}(M(\Gamma))$ and $K_{\mathbf{H}}(M(\mathcal{I}(\Gamma)))$.

Lemma 6.6. Let $\left(\Gamma, v_{0}\right)$ be a strongly connected A-automaton. Then, again using $[p]$ for the class of a state in $\mathcal{I}\left(\Gamma, v_{0}\right)$, there exists $m \in K_{\mathbf{H}}(M(\mathcal{I}(\Gamma)))$ with $[p] m=[q]$ if and only if there exists $r \in K_{\mathbf{H}}(M(\Gamma))$ with $p r=q$.

Proof. We let $M=M(\Gamma)$ and $I=M(\mathcal{I}(\Gamma))$. Suppose $[p] m=[q]$ with $m \in K_{\mathbf{H}}(I)$. Let $G \in \mathbf{H}$ be an $A$-generated group such that $r \in K_{\mathbf{H}}(M)$ if and if $r \in 1 \mu^{-1}$ under the canonical relational morphism $\mu: M \bullet G$. Then, since $m \in K_{\mathbf{H}}(I)$, there exists $w \in \tilde{A}^{*}$ such that $[w]_{I}=m$ and $[w]_{G}=1$. Hence $[p] w=[q]$, and so, by Proposition $\left[6.3\right.$, there exists $\underline{w} \in F^{\omega-1}(A)$ such that $p \underline{w}=q$ and $[\underline{w}]_{F G(A)}=$ $[w]_{F G(A)}$. Therefore, $[\underline{w}]_{G}=[w]_{G}=1$, and $r=[\underline{w}]_{M} \in K_{\mathbf{H}}(M)$ with $p r=q$.

Conversely, suppose such an $r$ exists. Let $G \in \mathbf{H}$ be an $A$-generated group such that $m \in K_{\mathbf{H}}(I)$ if and only if there exists $w \in \tilde{A}^{*}$ such that $[w]_{I}=m$ and $[w]_{G}=1$ (that is, $m$ relates to 1 under the canonical relational morphism). Since $r \in K_{\mathbf{H}}(M)$, there exists $u \in A^{*}$ with $[u]_{M}=r$ and $[u]_{G}=1$. But then $p u=q$, so $[p] u=[q]$, and $m=[u]_{I} \in K_{\mathbf{H}}(I)$ as desired.

Proposition 6.7. Let $\left(\Gamma, v_{0}\right)$ be a strongly connected A-automaton. Then the following are equivalent:

1. $\Gamma$ is an inverse A-graph and $M(\mathcal{I}(\Gamma)) \in \mathbf{S l} m \mathbf{H}$;

2. $M(\Gamma) \in \mathbf{S l}(m \mathbf{H}$;

3. $M(\Gamma) \in \mathbf{R}: \mathbf{H}$.

Proof. Let $M=M(\Gamma)$ and $I=M(\mathcal{I}(\Gamma))$. If $\Gamma$ is an inverse $A$-graph, then $M \subseteq I$ and so if, in addition, $I \in \mathbf{S l} m \mathbf{H}$, then $M$ is as well. Clearly $\mathbf{S l} m \mathbf{H} \subseteq \mathbf{R} m \mathbf{H}$. Suppose $M \in \mathbf{R}\left(m \mathbf{H}\right.$. Then, since $E(M) \subseteq K_{\mathbf{H}}(M), M \in \mathbf{E R}$ whence $\Gamma$ is an inverse $A$-graph by Proposition 6.5. Suppose $p \sim_{\mathbf{H}} q$ in $\mathcal{I}(\Gamma)$. Then there exists $m_{1}, m_{2} \in K_{\mathbf{H}}(I)$ such that $p m_{1}=q$ and $q m_{2}=p$. But then, by the above lemma, there exist $r_{1}, r_{2} \in K_{\mathbf{H}}(M)$ such that $p r_{1}=q$ and $q r_{2}=p$. Now $\left(r_{1} r_{2}\right)^{\omega} r_{1}$ and $\left(r_{1} r_{2}\right)^{\omega}$ are $\mathcal{R}$-related elements of $K_{\mathbf{H}}(M)$ and hence equal. Thus

$$
p=p\left(r_{1} r_{2}\right)^{\omega}=p\left(r_{1} r_{2}\right)^{\omega} r_{1}=q .
$$

We can therefore conclude that $\mathcal{I}\left(\Gamma, v_{0}\right)$ is $\mathbf{H}$-extendible, and so $I \in \mathbf{S l}(m) \mathbf{H}$.

We note that the hypothesis that $\Gamma$ is strongly connected is needed in the above proposition. For instance, let $\mathbf{G}_{c o m}$ be the pseudovariety of all finite abelian groups,

$$
M=\left\langle x, y \mid x^{2}=y^{2}=x y x=y x y=0\right\rangle,
$$


and $\Gamma$ be the subgraph of $\Gamma_{\{x, y\}}(M)$ obtained by removing the vertex 0 ,

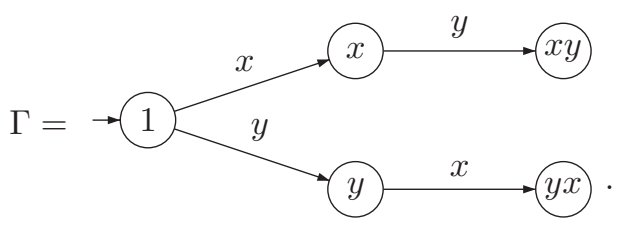

Then $\Gamma$ is an inverse $A$-graph, $M(\Gamma)=M \in \mathbf{S l} m \mathbf{G}_{c o m}$ (consider the relational morphism with $\mathbb{Z} / 3 \mathbb{Z}$ induced by sending $x, y$ to 1$)$, but $\mathcal{I}(\Gamma)$ is clearly not $\mathbf{G}_{\text {com }^{-}}$ extendible.

We now look at the special case where $\mathbf{H}=\mathbf{1}$, the trivial pseudovariety. Let $\mathbf{K}=\llbracket x^{\omega} y=x^{\omega} \rrbracket$ be the pseudovariety of all finite semigroups satisfying the above unary semigroup identity.

Proposition 6.8. Let $\left(\Gamma, v_{0}\right)$ be a strongly connected A-automaton. Then the following are equivalent:

1. $\Gamma$ has one vertex;

2. $M(\Gamma) \in \mathbf{S l}$;

3. $M(\Gamma) \in \mathbf{K}: \mathbf{S l}$.

Proof. Let $M=M(\Gamma)$. If $\Gamma$ has one vertex, then $\mathcal{I}(\Gamma)$ is 1-extendible and so $M \in \mathbf{S l}$. Clearly $\mathbf{S l} \subseteq \mathbf{K}(m)$ Sl. Suppose $M \in \mathbf{K} m \mathbf{S}$ Sl. Let $q \in V(\Gamma)$. Since $\left(\Gamma, v_{0}\right)$ is strongly connected, there exist $t, u \in A^{*}$ such that $v_{0} t=q, q u=v_{0}$. Then, since in any semilattice $(t u)^{\omega}=(t u)^{\omega} t,\left[(t u)^{\omega}\right]_{M}\left[(t u)^{\omega} t\right]_{M}=\left[(t u)^{\omega}\right]_{M}$. So

$$
q=v_{0}(t u)^{\omega}(t u)^{\omega} t=v_{0}(t u)^{\omega}=v_{0}
$$

whence $\Gamma$ has one vertex.

Now for our main theorem of this subsection.

Theorem 6.9. Let $\left(\Gamma, v_{0}\right)$ be a strongly connected A-automaton. Then the least congruence $\sim_{\mathbf{H}}$ on $\left(\Gamma, v_{0}\right)$ with quotient having transition monoid in $\mathbf{S l} \rightarrow \mathbf{H}$ is the congruence associated to the quotient $\mathcal{I}_{\mathbf{H}}\left(\Gamma, v_{0}\right)$. This congruence is given by $p \sim_{\mathbf{H}} q$ if and only if there exists $r \in K_{\mathbf{H}}(M(\Gamma))$ such that $p r=q$.

Proof. Let $M=M(\Gamma)$ and $I=M(\mathcal{I}(\Gamma))$. By Proposition 6.7 it is clear that the least such congruence is the one associated to $\mathcal{I}_{\mathbf{H}}\left(\Gamma, v_{0}\right)$. We use $[p]$ for the equivalence class of a state $p \in V(\Gamma)$ in $\mathcal{I}(\Gamma)$. Then $[p] \sim_{\mathbf{H}}[q]$ if and only if there exists $m \in K_{\mathbf{H}}(I)$ such that $[p] m=[q]$. But, by Lemma 6.6 this occurs if and only if there exists $r \in K_{\mathbf{H}}(M)$ such that $p r=q$. The result follows.

6.2. A Topological Viewpoint. Let $L \subseteq A^{*}$. Then $L$ is called a rational or recognizable language if there is an $A$-automaton $\left(\Gamma, v_{0}\right)$ and a subset $F \subseteq V(\Gamma)$, called the set of final states, such that

$$
L=\left\{w \in A^{*} \mid v_{0} w \in F\right\} .
$$

We say, in this case, that $\left(\Gamma, v_{0}\right)$ recognizes $L$. If $\left(\Gamma, v_{0}\right)$ is any automaton and $p, q \in V(\Gamma)$, we let

$$
L_{p, q}=\left\{w \in A^{*} \mid p w=q\right\} .
$$


This language is evidently rational. We call a rational language bonded if there exists a strongly connected $A$-automaton which recognizes it. The following proposition follows from Theorem 6.9 exactly as the analogous result followed for inverse automata.

Proposition 6.10. Let $\left(\Gamma, v_{0}\right)$ be a strongly connected $A$-automaton and $\mathbf{H}$ a pseudovariety of groups. Let $p, q \in V(\Gamma)$. Then $p \sim_{\mathbf{H}} q$ if and only if $1 \in \operatorname{cl}_{\mathbf{H}}\left(L_{p, q}\right) \subseteq$ $F G(A)$.

We now give a simple description, for a bonded language $L$, of its pro- $\mathbf{G}$ closure. In particular, we shall see that given a strongly connected $A$-automaton recognizing $L$, one can calculate $c l_{\mathbf{G}}(L)$ in rapid polynomial time (in the size of the automaton). First note that if $F \subseteq V(\Gamma)$ is the set of final states, then $L=\bigcup_{q \in F} L_{v_{0}, q}$ whence $c l_{\mathbf{G}}(L)=\bigcup_{q \in F} c l_{\mathbf{G}}\left(L_{v_{0}, q}\right)$. We are thus reduced to the case of a bonded language of the form $L_{v_{0}, q}$. We call such a language a simple bonded language. Later, we shall see that any rational language can be built up from singletons and simple bonded languages.

First, note the following fact. Let $\underline{w} \in F^{\omega-1}(A)$. Recall that $\rho: F^{\omega-1}(A) \rightarrow$ $F G(A)$ denotes the canonical surjection. Let $w_{n} \in A^{*}$ be obtained by replacing all the $\omega-1$ 's in $\underline{w}$ by $2 n !-1$. Then in any finite monoid (with the discrete topology), this sequence converges to $[\underline{w}]_{M}$. By considering the case that $M$ is a group, we see that $\left\{w_{n}\right\}$ converges to $[\underline{w}]_{F G(A)}$ in the pro-G topology.

Theorem 6.11. Let $\left(\Gamma, v_{0}\right)$ be a strongly connected A-automaton and $q \in V(\Gamma)$. Then

$$
c l_{\mathbf{G}}\left(L_{v_{0}, q}\right)=\pi_{1}\left(\mathcal{I}(\Gamma),\left[v_{0}\right]\right) u
$$

where $\left[v_{0}\right]$ is the equivalence class of $v_{0}$ in $V(\mathcal{I}(\Gamma))$ and $u \in \tilde{A}^{*}$ is any reduced word with $\left[v_{0}\right] u=[q]$. Furthermore, if

$$
L_{v_{0}, q}^{\prime}=\left\{\underline{w} \in F^{\omega-1}(A) \mid v_{0} \underline{w}=q\right\},
$$

then $\operatorname{cl}_{\mathbf{G}}\left(L_{v_{0}, q}\right)=L_{v_{0}, q}^{\prime} \rho$.

Proof. Let $M=M(\Gamma)$ and $H=\pi_{1}\left(\mathcal{I}(\Gamma), v_{0}\right)$. Suppose first that $\underline{w} \in L_{v_{0}, q}^{\prime}$, and let $\left\{w_{n}\right\}$ be the sequence associated to $\underline{w}$ as above. For $n \geq|M|,\left[w_{n}\right]_{M}=[\underline{w}]_{M}$, so $w_{n} \in L_{v_{0}, q}$ for $n$ large enough. But $\left\{w_{n}\right\}$ converges to $[\underline{w}]_{F G(A)}$, so $L_{v_{0}, q}^{\prime} \rho \subseteq$ $c l_{\mathbf{G}}\left(L_{v_{0}, q}\right)$. Suppose $w$ is a reduced word in $c l_{\mathbf{G}}\left(L_{v_{0}, q}\right)$. Then, since $F G(A)$ is metric in the pro-G topology, there exists a sequence $\left\{w_{n}\right\}$ of words in $L_{v_{0}, q}$ converging to $w$. Now since $v_{0} w_{n}=q,\left[v_{0}\right] w_{n}=[q]$ in $\mathcal{I}(\Gamma)$ whence, for all $n, w_{n} u^{-1} \in H$. But $H$ is a finitely generated subgroup, hence closed by Hall's Theorem [23]. Thus $w u^{-1} \in$ $H$, and so $w \in H u$ as desired. Suppose that $w \in H u$. Then $\left[v_{0}\right] w=\left[v_{0}\right] u=[q]$. Hence, by Proposition 6.3, there is a term $\underline{w} \in F^{\omega-1}(A)$ of height at most one such that $v_{0} \underline{w}=q$ and $[\underline{w}]_{F G(A)}=w$. The results follows.

Some comments are in order. First observe that if $L_{v_{0}, q}^{\prime \prime}$ is the collection of $(\omega-1)$-terms in $L_{v_{0}, q}^{\prime}$ of height at most one, then the above proof shows that $c l_{\mathbf{G}}\left(L_{v_{0}, q}\right)=L_{v_{0}, q}^{\prime \prime} \rho$. We note that, in particular,

$$
\pi_{1}\left(\mathcal{I}(\Gamma), v_{0}\right)=L_{v_{0}, v_{0}}^{\prime} \rho=\operatorname{cl}_{\mathbf{G}}\left(L_{v_{0}, v_{0}}\right) .
$$

Also observe that if we make $\mathcal{I}\left(\Gamma, v_{0}\right)$ an $\tilde{A}$-automaton in the obvious way, then the above theorem says that $c l_{\mathbf{G}}\left(L_{v, q}\right)$ is precisely the set of reduced words in $L_{\left[v_{0}\right],[q]}$ 
and, moreover, the reduction of any word in $L_{\left[v_{0}\right],[q]}$ is in the closure. More generally, if $F$ is any set of final states and $L$ the associated rational language, then $\operatorname{cl}_{\mathbf{G}}(L)$ consists of those reduced words recognized by $\mathcal{I}\left(\Gamma, v_{0}\right)$, viewed as an $\tilde{A}$-automaton, with set of final states $F^{\prime}=\{[q] \mid q \in F\}$. Moreover, the reduction of any accepted word is in the closure. Since $\mathcal{I}\left(\Gamma, v_{0}\right)$ is constructible in rapid polynomial time, the membership problem for the pro- $\mathbf{G}$ closure of a bonded rational language is computable in rapid polynomial time.

If $X \subseteq F G(A)$ and $\mathbf{H}, \mathbf{H}^{\prime}$ are pseudovarieties of groups with $\mathbf{H} \subseteq \mathbf{H}^{\prime}$, then it easily follows that $c l_{\mathbf{H}^{\prime}}(X) \subseteq c l_{\mathbf{H}}(X)$ whence $c l_{\mathbf{H}}(X)=\operatorname{cl}_{\mathbf{H}}\left(c l_{\mathbf{H}^{\prime}}(X)\right)$. We then have the following corollary.

Corollary 6.12. Suppose that $\left(\Gamma, v_{0}\right)$ is a strongly connected A-automaton, $q \in$ $V(\Gamma)$, and $\mathbf{H}$ is a pseudovariety of groups. Then

$$
c l_{\mathbf{H}}\left(L_{v_{0}, q}\right)=c l_{\mathbf{H}}\left(\pi_{1}\left(\mathcal{I}(\Gamma),\left[v_{0}\right]\right)\right) u
$$

where $\left[v_{0}\right]$ is the equivalence class of $v_{0}$ in $V(\mathcal{I}(\Gamma))$ and $u \in \tilde{A}^{*}$ is any reduced word with $\left[v_{0}\right] u=[q]$.

Proof. We have $c l_{\mathbf{H}}\left(L_{v_{0}, q}\right)=c l_{\mathbf{H}}\left(c l_{\mathbf{G}}\left(L_{v_{0}, q}\right)\right)=c l_{\mathbf{H}}\left(\pi_{1}\left(\mathcal{I}(\Gamma),\left[v_{0}\right]\right)\right) u$.

Thus if we can compute membership in the closure of a finitely generated subgroup of $F G(A)$ in the pro-H topology, as we can for $\mathbf{G}_{p}$ and $\mathbf{G}_{n i l}$, then we can compute the closure of a bonded rational language in that topology. We note the following very important corollary.

Corollary 6.13. Let $\mathbf{H}$ be an extension-closed pseudovariety of groups, $\left(\Gamma, v_{0}\right)$ a strongly connected $A$-automaton such that $\mathcal{I}\left(\Gamma, v_{0}\right)$ is $\mathbf{H}$-extendible, and $L$ a language recognized by $\Gamma$. Then $\operatorname{cl}_{\mathbf{H}}(L)=\operatorname{cl}_{\mathbf{G}}(L)$.

Proof. Let $H=\pi_{1}\left(\mathcal{I}(\Gamma),\left[v_{0}\right]\right)$. It suffices to consider a language of the form $L_{v_{0}, q}$. Since $\mathcal{I}\left(\Gamma, v_{0}\right)$ is $\mathbf{H}$-extendible, $H$ is a free factor in an open subgroup in the pro$\mathbf{H}$ topology whence, since $\mathbf{H}$ is extension-closed, $H$ is a closed subgroup. But $c l_{\mathbf{H}}\left(L_{v_{0}, q}\right)=c l_{\mathbf{H}}(H) u$ where $u \in \tilde{A}^{*}$ is a reduced word with $\left[v_{0}\right] u=[q]$. So $c l_{\mathbf{H}}\left(L_{v_{0}, q}\right)=H u=c l_{\mathbf{G}}\left(L_{v_{0}, q}\right)$.

Our next goal is to understand the relationship between the fundamental group of $\mathcal{I}_{\mathbf{H}}\left(\Gamma, v_{0}\right)$ and the closures of rational languages recognized by $\left(\Gamma, v_{0}\right)$.

Proposition 6.14. Let $\left(\Gamma, v_{0}\right)$ be a strongly connected A-automaton, $\mathbf{H}$ be a pseudovariety of groups, $H=\pi_{1}\left(\mathcal{I}(\Gamma),\left[v_{0}\right]\right)$, and $\tilde{H}=\pi_{1}\left(\mathcal{I}_{\mathbf{H}}(\Gamma),\left[v_{0}\right]_{\mathbf{H}}\right)$. Then $H \subseteq$ $\tilde{H} \subseteq c l_{\mathbf{H}}(H)$.

Proof. Since there is an automaton morphism from $\mathcal{I}\left(\Gamma, v_{0}\right)$ to $\mathcal{I}_{\mathbf{H}}\left(\Gamma, v_{0}\right)$, by Theorem $3.3 \subseteq \tilde{H}$. Since $c l_{\mathbf{H}}(H)=\bigcap_{\text {open } K \supseteq H} K$, to finish the proof it suffices to show that if $K$ is an open subgroup containing $H$, then $\tilde{H} \subseteq K$. Let $\Delta$ be the covering space of $B_{A}$ associated to $K$. Since $K$ has finite index, this covering space is finite-sheeted (in fact, it is just the coset graph of $F G(A) / K$ ). Since $K$ is open, $M(\Delta) \in \mathbf{H}$. Now since $H \subseteq K$ and $\Delta$ is a cover, by Theorem 3.3. there is an automaton morphism $\varphi:\left(\Gamma, v_{0}\right) \rightarrow \Delta$. Since $\Delta$ is trivially $\mathbf{H}$-extendible, by the universal property of $\mathcal{I}_{\mathbf{H}}\left(\Gamma, v_{0}\right), \varphi$ factors through $\mathcal{I}_{\mathbf{H}}\left(\Gamma, v_{0}\right)$, hence $\tilde{H} \subseteq K$.

Corollary 6.15. Let $\mathbf{H}$ be an extension-closed pseudovariety of groups, $\left(\Gamma, v_{0}\right)$ be a strongly connected A-automaton, $H=\pi_{1}\left(\mathcal{I}(\Gamma),\left[v_{0}\right]\right)$, and $\tilde{H}=\pi_{1}\left(\mathcal{I}_{\mathbf{H}}(\Gamma),\left[v_{0}\right]_{\mathbf{H}}\right)$. Then $c l_{\mathbf{H}}(H)=\tilde{H}$. 
Proof. Since $\mathbf{H}$ is extension-closed and $\mathcal{I}_{\mathbf{H}}\left(\Gamma, v_{0}\right)$ is $\mathbf{H}$-extendible, $\tilde{H}$ is closed by Proposition 5.15. Hence, by the above proposition, $\tilde{H}=c l_{\mathbf{H}}(H)$.

This corollary shows that if $\mathbf{H}$ is extension-closed and there is an algorithm which, given a finite set of reduced words $Y$, determines membership in $c l_{\mathbf{H}}(\langle Y\rangle)$, then one can effectively obtain a finite generating set for $c l_{\mathbf{H}}(\langle Y\rangle)$. Just construct, by the Stallings algorithm, an inverse automaton $\left(\Gamma, v_{0}\right)$ with fundamental group $\langle Y\rangle$. Then, by hypothesis, one can effectively construct $\mathcal{I}_{\mathbf{H}}\left(\Gamma, v_{0}\right)$ whose fundamental group, by the above corollary, is the closure. One can then find a finite generating set. This argument also shows that, in the extension-closed setting, the membership problem for $\mathbf{S l ~} \mathrm{m} \mathbf{H}$ is equivalent to the existence of such an algorithm (since if we can decide membership in $\mathbf{S l ~} \mathbf{H} \mathbf{H}$, we can construct effectively $\left.\mathcal{I}_{\mathbf{H}}\left(\Gamma, v_{0}\right)\right)$.

We now obtain an alternate characterization of the $\mathbf{H}$-closure of a bonded rational language.

Proposition 6.16. Let $\left(\Gamma, v_{0}\right)$ be a strongly connected A-automaton, $q \in V(\Gamma)$, and $\mathbf{H}$ a pseudovariety of groups. Then

$$
c l_{\mathbf{H}}\left(L_{v_{0}, q}\right)=c l_{\mathbf{H}}\left(\pi_{1}\left(\mathcal{I}_{\mathbf{H}}(\Gamma),\left[v_{0}\right]_{\mathbf{H}}\right)\right) u
$$

where $\left[v_{0}\right]_{\mathbf{H}}$ is the equivalence class of $v_{0}$ in $V\left(\mathcal{I}_{\mathbf{H}}(\Gamma)\right)$ and $u \in \tilde{A}^{*}$ is any reduced word with $\left[v_{0}\right]_{\mathbf{H}} u=[q]_{\mathbf{H}}$.

Proof. Let $H=\pi_{1}\left(\mathcal{I}(\Gamma),\left[v_{0}\right]\right)$ and $\tilde{H}=\pi_{1}\left(\mathcal{I}_{\mathbf{H}}(\Gamma),\left[v_{0}\right]_{\mathbf{H}}\right)$. Then Proposition 6.14 shows that $c l_{\mathbf{H}}(H)=c l_{\mathbf{H}}(\tilde{H})$. So, by Corollary 6.12 it suffices to show that if $u^{\prime} \in \tilde{A}^{*}$ such that $\left[v_{0}\right] u^{\prime}=[q]$, then $u^{\prime} u^{-1} \in c l_{\mathbf{H}}(H)$. But $\left[v_{0}\right] u^{\prime}=[q]$ implies that $\left[v_{0}\right]_{\mathbf{H}} u^{\prime}=[q]_{\mathbf{H}}$, and hence $u^{\prime} u^{-1} \in \tilde{H} \subseteq c l_{\mathbf{H}}(H)$.

In the case that $\mathbf{H}$ is extension-closed, and so $\tilde{H}=c l_{\mathbf{H}}(H)$, we see that if a strongly connected automaton $\left(\Gamma, v_{0}\right)$ recognizes a language $L$ via final states $F$, then the set of reduced words recognized by $\mathcal{I}_{\mathbf{H}}\left(\Gamma, v_{0}\right)$, viewed as an $\tilde{A}^{*}$-automaton, with final states $F^{\prime}=\left\{[q]_{\mathbf{H}} \mid q \in F\right\}$ is $c l_{\mathbf{H}}(L)$, and the reduction of any word recognized by this automaton is in the $\mathbf{H}$-closure.

6.3. Some Remarks on Non-Deterministic Automata. Many of the results of the previous subsection generalize with little change to non-deterministic automata. Let $\left(\Gamma, v_{0}\right)$ be a non-deterministic $A$-automaton and $F \subseteq V(\Gamma)$. We say that $L \subseteq A^{*}$ is recognized by $\left(\Gamma, v_{0}\right)$ with final states $F$ if

$$
L=\left\{w \in A^{*} \mid w \text { has a run from } v_{0} \text { to } q \text { for some } q \in F\right\} .
$$

It is well known [14] that any language recognized by a non-deterministic automaton is rational. We now extend the definition of bonded and simple bonded languages to include rational languages recognized by strongly connected non-deterministic automata. For a non-deterministic automaton $\left(\Gamma, v_{0}\right)$, we define, for $p, q \in V(\Gamma)$, $L_{p, q}$ to be the set of all words with a run from $p$ to $q$.

Lemma 6.17. Let $\left(\Gamma, v_{0}\right)$ be a strongly connected, non-deterministic, A-automaton and suppose $p \stackrel{a}{\longrightarrow} q$ is an edge in $\Gamma$. Then $a^{-1} \in \operatorname{cl}_{\mathbf{G}}\left(L_{q, p}\right)$.

Proof. Let $t \in A^{*}$ such that $t$ runs from $q$ to $p$. Then $(t a)^{2 n !-1} t \in L_{q, p}$ for all $n>0$ and $(t a)^{2 n !-1} \rightarrow a^{-1}$ in $F G(A)$. 
Lemma 6.18. Let $\left(\Gamma, v_{0}\right)$ be a strongly connected, non-deterministic A-automaton and suppose $w \in \tilde{A}^{*}$ has a run from $p$ to $q$ with $p, q \in V(\Gamma)$. Then $[w]_{F G(A)} \in$ $c l_{\mathbf{G}}\left(L_{p, q}\right)$.

Proof. First note that if $r, s, t \in V(\Gamma)$, then $L_{r, s} L_{s, t} \subseteq L_{r, t}$; also, by continuity of multiplication, for $X, Y \subseteq F G(A), c l_{\mathbf{G}}(X) c l_{\mathbf{G}}(Y) \subseteq c l_{\mathbf{G}}(X Y)$. The result now follows from the above lemma.

In a manner similar to the proof of Proposition 6.3 one can prove the following.

Proposition 6.19. Let $\left(\Gamma, v_{0}\right)$ be a strongly connected, non-deterministic A-automaton, $p, q \in V(\Gamma)$, and suppose $w \in \tilde{A}^{*}$ is such that $[p] w=[q]$ where $[p],[q]$ are the equivalence classes of $p$ and $q$ in $\mathcal{I}\left(\Gamma, v_{0}\right)$. Then $[w]_{F G(A)} \in \operatorname{cl}_{\mathbf{G}}\left(L_{p, q}\right)$.

We now prove Theorem 6.11 for strongly connected, non-deterministic automata. All the subsequent remarks, corollaries, and propositions of the above subsection which do not involve $(\omega-1)$-terms then apply without change in this context and we shall use them freely.

Theorem 6.20. For $\left(\Gamma, v_{0}\right)$ a strongly connected, non-deterministic A-automaton and $q \in V(\Gamma)$,

$$
c l_{\mathbf{G}}\left(L_{v_{0}, q}\right)=\pi_{1}\left(\mathcal{I}(\Gamma),\left[v_{0}\right]\right) u
$$

where $\left[v_{0}\right]$ is the equivalence class of $v_{0}$ in $V(\mathcal{I}(\Gamma))$ and $u \in \tilde{A}^{*}$ is any reduced word with $\left[v_{0}\right] u=[q]$.

Proof. Let $H=\pi_{1}\left(\mathcal{I}(\Gamma),\left[v_{0}\right]\right)$. Suppose $w$ is a reduced word in $\operatorname{cl}_{\mathbf{G}}\left(L_{v_{0}, q}\right)$; then there exists a sequence $\left\{w_{n}\right\}$ of words in $L_{v_{0}, q}$ converging to $w$. Since each $w_{n}$ has a run from $v_{0}$ to $q$, it follows that $\left[v_{0}\right] w_{n}=[q]$. Thus $w_{n} u^{-1} \in H$ for all $n$ whence, by Hall's Theorem, $w \in H u$. Conversely, if $w \in H u$, then $\left[v_{0}\right] w=[q]$. So, by the above proposition, $w \in c l_{\mathbf{G}}\left(L_{v_{0}, q}\right)$.

For the next section, we shall need to use non-deterministic automata with $\varepsilon$ transitions. A labeling of a graph $\Gamma$ over $A$ can be thought of as a faithful "graph morphism" $\ell: \Gamma \rightarrow B_{A}^{*}$ such that $E^{+}(\Gamma) \ell \subseteq A$. A labeling with $\varepsilon$-transitions of a graph $\Gamma$ over $A$ is then a faithful "graph morphism" $\ell: \Gamma \rightarrow B_{A}^{*}$ such that $E^{+}(\Gamma) \ell \subseteq A \cup\{\varepsilon\}$ where $\varepsilon$ represents the empty path. A non-deterministic $A$ automaton with $\varepsilon$-transitions is then a pair $\left(\Gamma, v_{0}\right)$ where $\Gamma$ is a finite graph labeled over $A$ with $\varepsilon$-transitions and $v_{0} \in V(\Gamma)$. We say that $L \subseteq A^{*}$ is recognized by $\left(\Gamma, v_{0}\right)$ with set of final states $F$ if

$$
L=\left\{p \ell \mid p \text { is a path in } \Gamma \text { with } p \alpha=v_{0}, p \omega \in F\right\} .
$$

One can again show that such languages are rational [14].

6.4. General Automata. We now study properties of automata in terms of their strongly connected components. First our promised result on the decomposition of rational languages over $A$ into languages built up from simple bonded languages and singletons. We call an edge $e$ of a graph a transition edge if it is not part of a strongly connected component. Observe that the language consisting of the empty string is a simple bonded language. 
Theorem 6.21. Let $\left(\Gamma, v_{0}\right)$ be a non-deterministic A-automaton, $F$ a set of final states, and $L$ the rational language recognized by $\left(\Gamma, v_{0}\right)$ with these final states. Then

$$
L=\bigcup_{j=1}^{n} L_{j, 0} a_{j, 1} L_{j, 1} \cdots a_{j, n_{j}} L_{j, n_{j}}
$$

where the $L_{j, i}$ are simple bonded languages recognized by strongly connected components of $\Gamma$ and the $a_{j, i} \in A$. Moreover, this decomposition can be obtained effectively in polynomial time.

Proof. Since $L=\bigcup_{q \in F} L_{v_{0}, q}$, we just need to show that each language $L_{v_{0}, q}$ can effectively be written as a finite union of languages of the form $L_{0} a_{1} L_{1} \cdots a_{n} L_{n}$ with the $L_{j}$ simple bonded languages recognized by strongly connected components of $\left(\Gamma, v_{0}\right)$ and the $a_{j} \in A$. Consider the graph $\Delta$ obtained from $\Gamma$ by identifying all vertices in the same strongly connected component and then removing all loops. We use $[p]$ for the equivalence class of a state $p \in V(\Gamma)$. Then in $\Delta$, there are no paths of length greater than the number of vertices of $\Delta$, so $\Delta^{*}$ is a finite category. Define a "graph morphism" $\varphi: \Gamma \rightarrow \Delta^{*}$ by $p \varphi=[p]$ on vertices, $e \varphi=e$ (viewed as an edge in $\Delta$ ) for a transition edge $e$, and $e \varphi=1_{[e \alpha]}$ for an edge $e$ which is not a transition edge. There is then an induced map $\varphi: \Gamma^{*} \rightarrow \Delta^{*}$. Now $\Gamma^{*}\left(v_{0}, q\right) \varphi=\Delta^{*}\left(\left[v_{0}\right],[q]\right)$, a finite subset. Since, for $p, r \in V(\Gamma), L_{p, r}=\Gamma^{*}(p, r) \ell$ (where $\ell: \Gamma \rightarrow B_{A}$ is the labeling) and $\varphi$ merely erases those edges belonging to a strongly connected component, $L_{v_{0}, q}$ is the finite union of the sets

$$
L_{v_{0}, e_{1} \alpha}\left(e_{1} \ell\right) L_{e_{1} \omega, e_{2} \alpha}\left(e_{2} \ell\right) \cdots L_{e_{n-1} \omega, e_{n} \alpha}\left(e_{n} \ell\right) L_{e_{n} \omega, q}
$$

where $e_{1} \cdots e_{n}$ is a path from $\left[v_{0}\right]$ to $[q]$ in $\Delta^{*}$. Note that this construction is clearly polynomial time (and, in fact, quite quick).

Our first step in understanding the closure of a rational language is to compute the closure of a product of simple bonded languages and singletons.

Lemma 6.22. Let $\mathbf{H}$ be an extension-closed pseudovariety of groups, $A$ a finite set, $H_{0}, \ldots, H_{n}$ finitely generated closed subgroups of $F G(A)$, and $g_{0}, \ldots, g_{n+1} \in$ $F G(A)$. Then $g_{0} H_{0} g_{1} \cdots H_{n} g_{n+1}$ is closed.

Proof. Let $N_{i}=g_{n+1}^{-1} \cdots g_{i+1}^{-1} H_{i} g_{i+1} \cdots g_{n+1}$. Then, since conjugation is a continuous automorphism, each $N_{i}$ is a finitely generated closed subgroup. An easy verification shows that

$$
g_{0} H_{0} g_{1} \cdots H_{n} g_{n+1}=g_{0} \cdots g_{n+1} N_{0} N_{1} \cdots N_{n}
$$

So, by Theorem 5.19 and since right translation is a homeomorphism, we see that $g_{0} \cdots g_{n+1} N_{0} N_{1} \cdots N_{n}$ is closed. The result follows.

Corollary 6.23. Let $\mathbf{H}$ be an extension-closed pseudovariety of groups, $L_{0}, \cdots, L_{n}$ simple bonded languages over an alphabet $A$, and $a_{1}, \ldots, a_{n} \in A$. Then

$$
c l_{\mathbf{H}}\left(L_{0} a_{1} \cdots a_{n} L_{n}\right)=\operatorname{cl}_{\mathbf{H}}\left(L_{0}\right) a_{1} \cdots a_{n} c l_{\mathbf{H}}\left(L_{n}\right) .
$$

Furthermore, $c_{\mathbf{H}}\left(L_{0} a_{1} \cdots a_{n} L_{n}\right)$ can be put in the form $g N_{0} \cdots N_{n}$ for some finitely generated closed subgroups $N_{i}(i=0, \ldots, n)$. 
Proof. By continuity of multiplication, the right-hand side is contained in the lefthand side; so it suffices to show that the right-hand side is closed. Suppose $\left(\Gamma_{i}, v_{i}\right)$ is a non-deterministic, strongly connected automaton recognizing $L_{i}$. Then, by Corollary 6.15 $H_{i}=\pi_{1}\left(\mathcal{I}_{\mathbf{H}}\left(\Gamma_{i}\right),\left[v_{i}\right]_{\mathbf{H}}\right)$ is closed and, by Proposition 6.16], $c l_{\mathbf{H}}\left(L_{i}\right)=H_{i} w_{i}$ for an appropriately chosen $w_{i} \in F G(A)$. Hence the right-hand side is equal to $H_{0} w_{0} a_{1} \cdots a_{n} H_{n} w_{n}$ which is closed by Lemma 6.22. The last statement follows from the proof of Lemma 6.22.

An immediate consequence is the following.

Proposition 6.24. Let $L$ be a rational language over an alphabet $A$ and $\mathbf{H}$ an extension-closed pseudovariety of groups. Suppose

$$
L=\bigcup_{j=1}^{n} L_{j, 0} a_{j, 1} L_{j, 1} \cdots a_{j, n_{j}} L_{j, n_{j}}
$$

with the $L_{j, i}$ simple bonded languages and the $a_{j, i} \in A$. Then

$$
c l_{\mathbf{H}}(L)=\bigcup_{j=1}^{n} c l_{\mathbf{H}}\left(L_{j, 0}\right) a_{j, 1} \cdots a_{j, n} c l_{\mathbf{H}}\left(L_{j, n}\right) .
$$

Furthermore, $c l_{\mathbf{H}}(L)$ can be written as a finite union of sets of the form $g N_{0} \cdots N_{n}$ with $g \in F G(A)$ and the $N_{i}$ finitely generated closed subgroups.

We then obtain the following corollary.

Corollary 6.25. Let $L_{1}, L_{2}$ be rational languages over $A$ and $\mathbf{H}$ an extensionclosed pseudovariety of groups. Then

$$
c l_{\mathbf{H}}\left(L_{1} L_{2}\right)=c l_{\mathbf{H}}\left(L_{1}\right) c l_{\mathbf{H}}\left(L_{2}\right) .
$$

We are almost ready for the main result of this section. A version of this algorithm can be found in 38], although the idea is due to Pin and Reutenauer [34]. However, their algorithm requires the algorithm to find a finite set of generators for a subgroup generated by a rational set, while ours does not. Also, this algorithm is more transparent, as it is geometric in nature, and we shall see below that one can perform it by hand in reasonable situations. Most importantly, given a non-deterministic automaton, the algorithm constructs a non-deterministic $\tilde{A}$ automaton with $\varepsilon$-transitions which can be used to recognize the pro- $\mathbf{H}$ closure of any rational language recognized by the original automaton. First we need the Gilman $\varepsilon$-completion procedure [19] which proves a result of Benois [11.

Theorem 6.26. Let $L \subseteq \tilde{A}^{*}$ be a rational language and $\rho: \tilde{A}^{*} \rightarrow \tilde{A}^{*}$ the map which takes a word to its reduction. Then $L \rho$ is rational. Furthermore, given a nondeterministic $\tilde{A}$-automaton $\left(\Gamma, v_{0}\right)$ recognizing $L$, one can construct, in polynomial time in the size of $\Gamma$, a non-deterministic $\tilde{A}$-automaton with $\varepsilon$-transitions which recognizes $L \rho$.

Proof. We merely give the algorithm, as the result is well known. Let $\Gamma_{0}=\Gamma$. The procedure stops if, between any two vertices of $\Gamma_{i}$, there is an $\varepsilon$-transition in each direction. If not, one searches through the vertices of $\Gamma_{i}$ until one finds a pair of distinct vertices $u$ and $v$ such that, either there is a path from $u$ to $v$ of length 2 with label $\varepsilon$ or $a a^{-1}$ with $a \in \tilde{A}$, or such that there is a path from $u$ to $v$ of length 3 with label $a a^{-1}, a \in \tilde{A}$. If no such pair is found, the algorithm is terminated. 
Otherwise, one adds an $\varepsilon$-transition from $u$ to $v$ forming $\Gamma_{i+1}$ and iterates the procedure. Let $\Gamma^{\prime}$ be the resulting automaton. There is a well-known $\tilde{A}$-automaton $\Delta$ which recognizes precisely the reduced words of $\tilde{A}^{*}$. The desired automaton is then the connected component containing the initial state of the product of these two automata.

Theorem 6.27. Let $\left(\Gamma, v_{0}\right)$ be a non-deterministic A-automaton and $\mathbf{H}$ be an extension-closed pseudovariety. Let $\left(\Gamma^{\prime},\left[v_{0}\right]\right)$ be the $\tilde{A}$-automaton obtained from $\Gamma$ by replacing each strongly connected component $\Gamma_{j}$ with $\mathcal{I}_{\mathbf{H}}\left(\Gamma_{j}\right)$ where we only allow reverse transitions in the various $\mathcal{I}_{\mathbf{H}}\left(\Gamma_{j}\right)$. We use $[v]$ for the class of a vertex $v$. Let $F \subseteq V(\Gamma), F^{\prime}=\{[q] \mid q \in F\}$, and $L^{\prime}$ the language recognized by $\left(\Gamma^{\prime},\left[v_{0}\right]\right)$ with final states $F^{\prime}$. Then $L^{\prime} \rho=c l_{\mathbf{H}}(L)$. Hence performing the Gilman $\varepsilon$-completion procedure on $\left(\Gamma^{\prime},\left[v_{0}\right]\right)$ gives a non-deterministic $\tilde{A}$-automaton with $\varepsilon$-transitions which can be used to recognize the $\mathbf{H}$-closure of any language recognized by $\left(\Gamma, v_{0}\right)$.

Proof. Since taking the $\mathbf{H}$-closure commutes with finite unions, it suffices to show that if $q \in F$, then $L_{\left[v_{0}\right],[q]} \rho=c l_{\mathbf{H}}\left(L_{v_{0}, q}\right)$. Let $\Delta$ be the graph obtained from $\Gamma$, as above, by identifying the strongly connected components to points, and then removing the loops. We note that $\Delta$ is the same graph that one would obtain by performing this construction on $\Gamma^{\prime}$. Now arguing as before, $L_{v_{0}, q}$ is the finite union of the sets

$$
L_{v_{0}, e_{1} \alpha}\left(e_{1} \ell\right) L_{e_{1} \omega, e_{2} \alpha}\left(e_{2} \ell\right) \cdots L_{e_{n-1} \omega, e_{n} \alpha}\left(e_{n} \ell\right) L_{e_{n} \omega, q}
$$

over the finitely many paths $e_{1} \cdots e_{n}$ between the class of $v_{0}$ and the class of $q$ in $\Delta^{*}$. Similarly, $L_{\left[v_{0}\right],[q]}$ is the finite union of the sets

$$
L_{\left[v_{0}\right],\left[e_{1} \alpha\right]}\left(e_{1} \ell\right) L_{\left[e_{1} \omega\right],\left[e_{2} \alpha\right]}\left(e_{2} \ell\right) \cdots L_{\left[e_{n-1} \omega\right],\left[e_{n} \alpha\right]}\left(e_{n} \ell\right) L_{\left[e_{n} \omega\right],[q]}
$$

over the same set of paths $e_{1} \cdots e_{n}$ in $\Delta^{*}$. Now, by Proposition $6.24, c l_{\mathbf{H}}\left(L_{v_{0}, q}\right)$ is the finite union of the sets

$$
c l_{\mathbf{H}}\left(L_{v_{0}, e_{1} \alpha}\right)\left(e_{1} \ell\right) c l_{\mathbf{H}}\left(L_{e_{1} \omega, e_{2} \alpha}\right)\left(e_{2} \ell\right) \cdots c l_{\mathbf{H}}\left(L_{e_{n-1} \omega, e_{n} \alpha}\right)\left(e_{n} \ell\right) c l_{\mathbf{H}}\left(L_{e_{n} \omega, q}\right) .
$$

But, by the remarks at the end of subsection [6.2, $L_{\left[p^{\prime}\right],\left[q^{\prime}\right]} \rho=c l_{\mathbf{H}}\left(L_{p^{\prime}, q^{\prime}}\right)$ if $p^{\prime}$ and $q^{\prime}$ are in the same strongly connected component of $\Gamma$. Hence $L_{\left[v_{0}\right],[q]} \rho=c l_{\mathbf{H}}\left(L_{p, q}\right)$ and the result follows.

Theorem 6.28. Suppose that $\mathbf{H}$ is an extension-closed pseudovariety of groups, $A$ a finite set, and that there is an algorithm with complexity $f$ to compute membership in the closure of a finitely generated subgroup of $F G(A)$. Then, given a rational language $L$ over $A$ and a non-deterministic A-automaton $\left(\Gamma, v_{0}\right)$ recognizing $L$, there is an algorithm to compute membership in $c_{\mathbf{H}}(L)$ in only polynomially slower time than $f$. In addition, one can, within the same time bound, find elements $g_{j} \in F G(A)$ and finite sets of reduced words $X_{j, i}$ such that $\left\langle X_{j, i}\right\rangle$ is closed for all $j, i$ and

$$
c l_{\mathbf{H}}(L)=\bigcup_{j=1}^{n} g_{j}\left\langle X_{j, 0}\right\rangle \cdots\left\langle X_{j, n_{j}}\right\rangle .
$$

In particular, the algorithm for $\mathbf{G}$ is in rapid polynomial time.

Proof. Since $\mathcal{I}_{\mathbf{H}}\left(\Gamma_{j}\right)$ is computable for each strongly connected component $\Gamma_{j}$ of $\Gamma$ in only polynomially worse time than $f$ and the Gilman $\varepsilon$-completion process can be 
done in polynomial time, we can obtain a non-deterministic automaton recognizing $c l_{\mathbf{H}}(L)$ in only polynomially worse time than $f$ by the above theorem.

Furthermore, by Proposition 6.16] and since $\mathbf{H}$ is extension-closed, for $p, q \in$ $V\left(\Gamma_{j}\right), c l_{\mathbf{H}}\left(L_{[p],[q]}\right)$ is just the coset of $\pi_{1}\left(\mathcal{I}_{\mathbf{H}}\left(\Gamma_{j}\right),[p]\right)$ corresponding to any reduced word reading from $[p]$ to $[q]$, the penultimate statement follows from Theorem 6.21 Proposition 6.24, and of the proof of Lemma 6.22

Since, for $\mathbf{G}, \mathcal{I}_{\mathbf{G}}(\Gamma)=\mathcal{I}(\Gamma)$, the algorithm is quite fast indeed. We now give an example to show that if the automaton $\left(\Gamma, v_{0}\right)$ in question is of a reasonable size, one can actually perform this algorithm to find the G-closure by hand. Let $L$ be the language recognized by the following automaton.

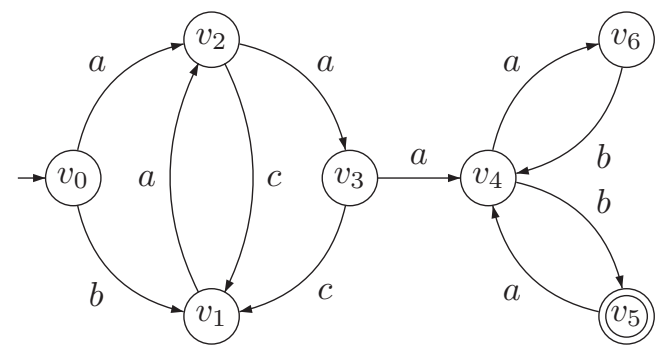

We now use our algorithm to find $c_{\mathbf{G}}(L)$ in $F G(\{a, b, c\})$. First we decompose $L$ as a finite union of products of singletons and finitely generated subgroups. When we identify the strongly connected components to points, we get the following graph.

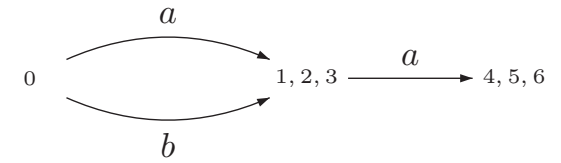

So $L=a L_{v_{2}, v_{3}} a L_{v_{4}, v_{5}} \cup b L_{v_{1}, v_{3}} a L_{v_{4}, v_{5}}=\left(a L_{v_{2}, v_{3}} \cup b L_{v_{1}, v_{3}}\right) a L_{v_{4}, v_{5}}$. Now when we fold the strongly connected component $\Omega$ of $\left\{v_{1}, v_{2}, v_{3}\right\}$, we obtain the following.
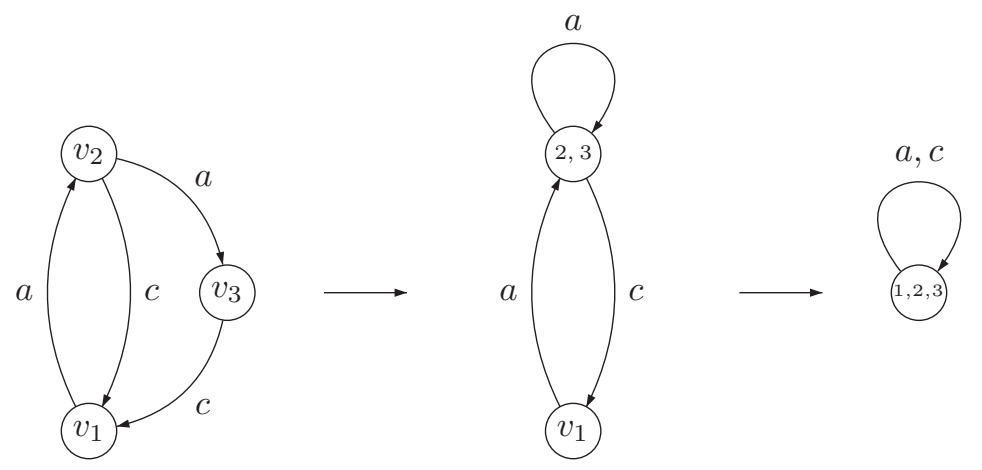

Hence $\pi_{1}\left(\mathcal{I}(\Omega),\left[v_{2}\right]\right)=\langle a, c\rangle=\pi_{1}\left(\mathcal{I}(\Omega),\left[v_{1}\right]\right)$. So

$$
c l_{\mathbf{G}}\left(L_{v_{2}, v_{3}}\right)=c l_{\mathbf{G}}\left(L_{v_{1}, v_{3}}\right)=\langle a, c\rangle .
$$

The strongly connected component $\Delta$ of $\left\{v_{4}, v_{5}, v_{6}\right\}$ is already an inverse $\{a, b, c\}$ graph. We choose $T$ as a maximal subtree. 

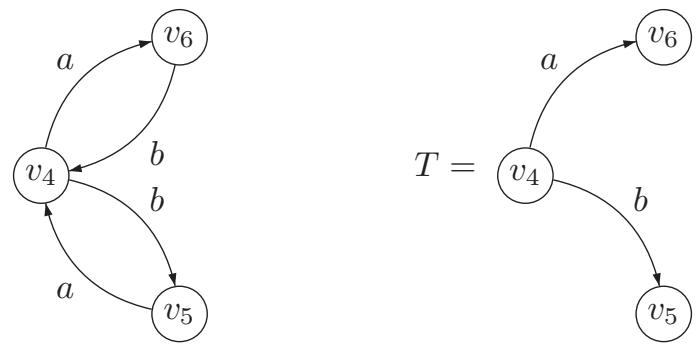

Then $\pi_{1}\left(\Delta, v_{4}\right)=\langle a b, b a\rangle$ and $v_{4} b=v_{5}$. So $c l_{\mathbf{G}}\left(L_{v_{4}, v_{5}}\right)=\langle a b, b a\rangle b$. Thus

$$
c l_{\mathbf{G}}(L)=(a\langle a, c\rangle \cup b\langle a, c\rangle) a\langle a b, b a\rangle b=(a \cup b)\langle a, c\rangle a\langle a b, b a\rangle b .
$$

Then conjugating as in the proof of Lemma [6.22, we see that

$$
c l_{\mathbf{G}}(L)=\left(a^{2} b \cup b a b\right)\left\langle b^{-1} a b, b^{-1} a^{-1} c a b\right\rangle\left\langle b^{-1} a b^{2}, a b\right\rangle .
$$

Finally, we get the automaton below whose $\varepsilon$-completion recognizes $c l_{\mathbf{G}}(L)$.

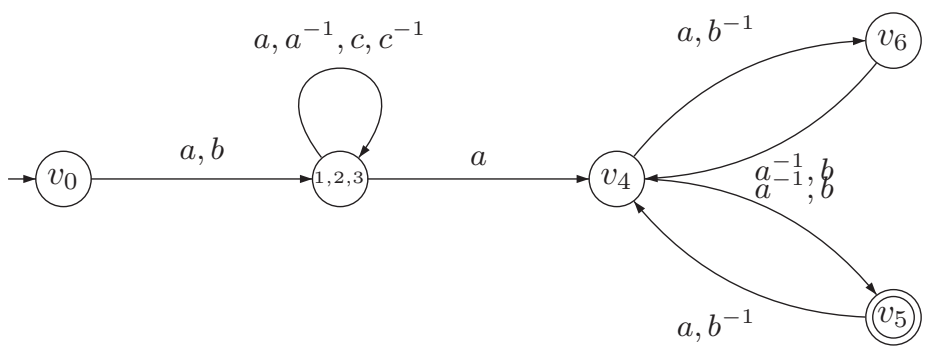

This algorithm also allows simple calculation of $K_{\mathrm{G}}(M)$ of a monoid. Indeed, one just needs to fold all the strongly connected components of $\Gamma_{A}(M)$ to make them inverse $A$-graphs (one can even do this in parallel); then one adds the reverse transitions for these components; finally, one performs the Gilman $\varepsilon$-completion procedure. All of this can be done in polynomial time. Then, in parallel, one can check for each element $m$ whether there is a path labeled by $\varepsilon$ from the class of 1 to the class of $m$. This is probably more efficient then generating the least submonoid closed under weak conjugation [24]. To compute $K_{\mathbf{G}_{p}}(M)$, one would add the extra, polynomial time step of computing $\mathcal{I}_{\mathbf{H}}(\Delta)$ for each strongly connected component.

Using Theorem 6.11 and Proposition 6.24 we obtain the following result which implies the Type II Conjecture of Rhodes, $\mathbf{J} * \mathbf{G}=\mathbf{J}(m \mathbf{G}$, and a great deal more; see [4, 5, 13].

Theorem 6.29. Let $\left(\Gamma, v_{0}\right)$ be an A-automaton, $q \in V(\Gamma)$, and

$$
L_{p, q}^{\prime}=\left\{\underline{w} \in F^{\omega-1}(A) \mid v_{0} \underline{w}=q\right\} .
$$

Then $c_{\mathbf{G}}\left(L_{v_{0}, q}\right)=L_{p, q}^{\prime} \rho$. Again, one could restrict to terms of height at most one.

Let $\mathbf{H}$ be a pseudovariety of groups and $\left(\Gamma, v_{0}\right)$ a non-deterministic $A$-automaton. We then call $\left(\Gamma, v_{0}\right)$ locally $\mathbf{H}$-extendible if the strongly connected components of $\left(\Gamma, v_{0}\right)$ are all $\mathbf{H}$-extendible inverse $A$-graphs. Let $\Delta$ be a strongly connected component of $\left(\Gamma, v_{0}\right)$. Then if $\left(\Gamma, v_{0}\right)$ is deterministic, $M(\Gamma)$ maps onto $M(\Delta)$, so Propositions 6.5 and 6.7 imply the following theorem. 
Theorem 6.30. Let $\left(\Gamma, v_{0}\right)$ be an A-automaton, then $\left(\Gamma, v_{0}\right)$ is locally $\mathbf{H}$-extendible if and only if, for each strongly connected component $\Delta, M(\Delta) \in \mathbf{S l} \rightarrow \mathbf{H}$. So, in particular, if $M(\Gamma) \in \mathbf{R} m \mathbf{H}$, then $\left(\Gamma, v_{0}\right)$ is locally $\mathbf{H}$-extendible. Also if $M(\Gamma) \in$ $\mathbf{E R}$, then $\left(\Gamma, v_{0}\right)$ is locally $\mathbf{G}$-extendible, that is, the strongly connected components are inverse A-graphs.

We now obtain the following important result generalizing Corollary 6.13.

Theorem 6.31. Let $\mathbf{H}$ be an extension-closed pseudovariety, $\left(\Gamma, v_{0}\right)$ a locally $\mathbf{H}-$ extendible (possibly non-deterministic) A-automaton, and $L$ a rational language recognized by $\left(\Gamma, v_{0}\right)$. Then $c_{\mathbf{G}}(L)=c l_{\mathbf{H}}(L)$. In particular, if $L$ is a rational language over $A$, recognized by an $A$-automaton with transition monoid in $\mathbf{R} \rightarrow \mathbf{H}$, then $\operatorname{cl}_{\mathbf{G}}(L)=\operatorname{cl}_{\mathbf{H}}(L)$.

Proof. By the above results, we see that $L$ can be expressed as a finite union of languages of the form $L_{0} a_{1} L_{1} \cdots a_{n} L_{n}$ with the $L_{j}$ recognized by strongly connected components of $\left(\Gamma, v_{0}\right)$. By Corollary 6.25,

$$
c l_{\mathbf{H}}\left(L_{0} a_{1} \cdots a_{n} L_{n}\right)=c l_{\mathbf{H}}\left(L_{0}\right) a_{1} \cdots a_{n} c l_{\mathbf{H}}\left(L_{n}\right) .
$$

The result now follows from Corollary 6.13 since taking the closure commutes with taking a finite union.

\section{Applications to Monoid Theory}

In this section, we finally get to apply our results to monoid theory. In particular, we obtain a short proof that $\mathbf{J}: \mathbf{H}=\mathbf{J} * \mathbf{H}$ for $\mathbf{H}$ extension-closed.

7.1. Mal'cev Products. We begin by characterizing algebraically those monoids whose right (and/or left) Cayley graphs are locally $\mathbf{H}$-extendible. As usual, dual results will be left to the reader. First we need the following elementary result. For a finite monoid $M$, we use, as before, $\oplus S c h(M)$ to denote the direct product of the monoids $S \operatorname{ch}(X)$ as $X$ ranges over the regular $\mathcal{R}$-classes and $\oplus \operatorname{Sch}^{\rho}(M)$ for the corresponding direct product of of $S \operatorname{ch}^{\rho}(Y)$ over regular $\mathcal{L}$-classes $Y$. Let $\mathbf{K}=$ $\llbracket x^{\omega} y=x^{\omega} \rrbracket, \mathbf{D}=\llbracket x y^{\omega}=y^{\omega} \rrbracket$, and $\mathbf{N}=\llbracket x^{\omega}=0 \rrbracket=\mathbf{K} \cap \mathbf{D}$ be the pseudovarieties of semigroups defined by the above unary semigroup identities. Recall that, for a morphism $\varphi$, we use $V_{\varphi}$ for its Mal'cev kernel.

Lemma 7.1. Let $M$ be a finite monoid.

1. Let $\varphi: M \rightarrow \oplus \operatorname{Sch}(M)$ be the natural map, then $V_{\varphi} \in \mathbf{K}$.

2. Let $\varphi^{\rho}: M \rightarrow \oplus S \operatorname{Sh}^{\rho}(M)$ be the natural map, then $V_{\varphi^{\rho}} \in \mathbf{D}$.

3. Let $\varphi \times \varphi^{\rho}: M \rightarrow \oplus S \operatorname{ch}(M) \times \oplus S \operatorname{ch}^{\rho}(M)$ be the natural map, then $V_{\varphi \times \varphi^{\rho}} \in \mathbf{N}$.

Proof. We prove just 1, 2 being dual and 3 following from 1 and 2 . Let $x, y \in M$ such that $x \varphi=y \varphi=(x \varphi)^{2}$. Then $x^{\omega} \varphi=x \varphi=y \varphi$. Let $X$ be the $\mathcal{R}$-class of $x^{\omega}$ and $q=x^{\omega}$. Then, since $x^{\omega} x^{\omega}=x^{\omega}, x^{\omega} \varphi=y \varphi$ implies $q x^{\omega}=q y$ whence $x^{\omega} y=x^{\omega}$ as desired.

We now give a geometric proof of the following well-known lemma.

Lemma 7.2. $\mathbf{K} \rightarrow \mathbf{S l}=\mathbf{R}, \mathbf{D} \rightarrow \mathbf{S l}=\mathbf{L}$, and $\mathbf{N} \rightarrow \mathbf{S l}=\mathbf{J}$.

Proof. We only prove $\mathbf{K} \rightarrow \mathbf{S l}=\mathbf{R}$. Let $M$ be a finite $A$-generated monoid in $\mathbf{K}(m \mathbf{S l}$. Then $S \operatorname{ch}(X) \in \mathbf{K}: \mathbf{S l}$ for every $\mathcal{R}$-class $X$. But $S \operatorname{ch}(X)$ is the transition monoid of the strongly connected $A$-automaton $S c h_{A}(X)$ (the base point is irrelevant), so, 
by Proposition 6.8, $S c h_{A}(X)$ has one vertex, and hence $M$ is $\mathcal{R}$-trivial. Conversely, if $M \in \mathbf{R}$, then, for each regular $\mathcal{R}$-class $X, S c h_{A}(X)$ has one vertex, so, by Proposition 6.8, $\operatorname{Sch}(X) \in \mathbf{S l}$ whence, by the above lemma, $M \in \mathbf{K} m \mathbf{S l}$.

We now characterize those monoids whose Schützenberger graphs are inverse.

Theorem 7.3. Let $M$ be a finite A-generated monoid. Let $\Gamma_{A}(M)$ be the right Cayley graph of $M$ and $\Gamma_{A}^{\rho}(M)$ be the left Cayley graph of $M$.

1. $M \in \mathbf{E R}$ if and only if $\left(\Gamma_{A}(M), 1\right)$ is locally $\mathbf{G}$-extendible, if and only if $S_{c h}(X)$ is an inverse A-graph for each regular $\mathcal{R}$-class $X$, if and only if $\oplus \operatorname{Sch}(M) \in \mathbf{E S l}$.

2. $M \in \mathbf{E L}$ if and only if $\left(\Gamma_{A}^{\rho}(M), 1\right)$ is locally $\mathbf{G}$-extendible, if and only if $S_{c h}^{\rho}(Y)$ is an inverse A-graph for each regular $\mathcal{L}$-class $Y$, if and only if $\oplus \operatorname{Sch}^{\rho}(M) \in \mathbf{E S l}$.

3. $M \in \mathbf{E J}$ if and only if 1 and 2 hold.

Proof. We just prove 1. By Theorem 6.30, we see that if $M \in \mathbf{E R}$, then $\Gamma_{A}(M)$ is locally G-extendible. Clearly if $\Gamma_{A}(M)$ is locally G-extendible, then, for each regular $\mathcal{R}$-class $X, S c h_{A}(X)$ is inverse. If $S c h_{A}(X)$ is an inverse $A$-graph for $X$ a regular $\mathcal{R}$-class, then, by Proposition 6.5, $S \operatorname{ch}(X) \in$ ESl. Therefore, $S \operatorname{Sch}_{A}(X)$ being inverse for every regular $\mathcal{R}$-class $X$ implies $\oplus S \operatorname{ch}(M) \in \mathbf{E S l}$. Since $\varphi$ : $\langle E(M)\rangle \rightarrow\langle E(\oplus S \operatorname{ch}(M))\rangle$, we see that if $\oplus S \operatorname{ch}(M) \in \mathbf{E S L}$, then $\langle E(M)\rangle \in \mathbf{K}(m)$ $\mathbf{S l}=\mathbf{R}$, so $M \in \mathbf{E R}$.

Observe that if $e, f \in E(M)$ are $\mathcal{R}$-equivalent, then $e f=f$ and $f e=e$. So, for monoids in ER, each $S c h_{A}(X)$, for $X$ regular, has a unique idempotent; we always take this idempotent as the initial state to make $S c h_{A}(X)$ a strongly connected $A$ automaton. In particular, if $\mathbf{H}$ is any pseudovariety of groups, then $\mathbf{R} m \mathbf{H} \subseteq \mathbf{E R}$ implies we are in the above situation for monoids in $\mathbf{R} m \mathbf{H}$. Also note that the above theorem shows that the property of the Cayley graph being locally $\mathbf{G}$-extendible is independent of the choice of generators, that is, an invariant of the monoid. Now one of our main results.

Theorem 7.4. Let $M$ be a finite A-generated monoid and $\mathbf{H}$ a pseudovariety of groups. Let $\Gamma_{A}(M)$ be the right Cayley graph of $M$ and $\Gamma_{A}^{\rho}(M)$ be the left Cayley graph of $M$.

1. $M \in \mathbf{R} m \mathbf{H}$ if and only if $\left(\Gamma_{A}(M), 1\right)$ is locally $\mathbf{H}$-extendible, if and only if, for each regular $\mathcal{R}$-class $X, S \operatorname{Sh}_{A}(X)$ is an $\mathbf{H}$-extendible inverse $A$-graph, if and only if $\oplus S \operatorname{ch}(M) \in \mathbf{S l}(m) \mathbf{H}$.

2. $M \in \mathbf{L}\left(m \mathbf{H}\right.$ if and only if $\left(\Gamma_{A}^{\rho}(M), 1\right)$ is locally $\mathbf{H}$-extendible, if and only if, for each regular $\mathcal{L}$-class $Y, S \operatorname{Sh}_{A}^{\rho}(Y)$ is an $\mathbf{H}$-extendible inverse A-graph, if and only if $\oplus \operatorname{Sch}^{\rho}(M) \in \mathbf{S l} \rightarrow \mathbf{H}$.

3. $M \in \mathbf{J}(\mathrm{H} \mathbf{H}$ if and only if both 1 and 2 hold.

Proof. Again we just prove 1. Since $\operatorname{Sch}_{A}(X)$ is strongly connected, the last two conditions are equivalent by Proposition 6.7. If $\oplus S \operatorname{ch}(M) \in \mathbf{S l} m \mathbf{H}$, then $M \in$

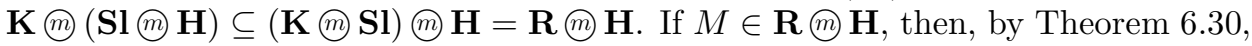
$\Gamma_{A}(M)$ is locally $\mathbf{H}$-extendible. But if $\Gamma_{A}(M)$ is locally $\mathbf{H}$-extendible, then, for each regular $\mathcal{R}$-class $X, S_{c h}(X)$ is an $\mathbf{H}$-extendible inverse $A$-graph.

This theorem gives a converse to Theorem 6.30 for the case of the Cayley graph of a monoid. We don't know whether the converse holds in general. Again, we 
see that the geometric property of the Cayley graph being locally $\mathbf{H}$-extendible is independent of the generating set and depends only on the regular $\mathcal{R}$-classes. We have the following corollary.

Corollary 7.5. Let $\mathbf{H}$ be a pseudovariety of groups.

1. $\mathbf{K} \rightarrow(\mathbf{S l} \rightarrow \mathbf{H})=(\mathbf{K} \rightarrow \mathbf{S l}) \oplus \mathbf{H}$.

2. $\mathbf{D} \rightarrow(\mathbf{S l} \rightarrow \mathbf{H})=(\mathbf{D} \rightarrow \mathbf{S l}) \rightarrow \mathbf{H}$.

3. $\mathbf{N}:(\mathbf{S l}: \mathbf{H})=(\mathbf{N}: \mathbf{S l}): \mathbf{H}$.

Recalling that $\mathbf{R}$ and $\mathbf{L}$ are local, we recover the following well known results, proved now by a geometric (rather than algebraic) argument.

Theorem 7.6. $\mathbf{E R}=\mathbf{R} * \mathbf{G}, \mathbf{E L}=\mathbf{L} * \mathbf{G}$, and $\mathbf{E} \mathbf{J}=\mathbf{J} \oplus \mathbf{G}$.

Let $\mathbf{B G}$ be the pseudovariety of block groups. It is not difficult to see that $\mathbf{B G}=\mathbf{E J}$. Indeed, if $M \in \mathbf{E J}$, then each regular $\mathcal{R}$ and $\mathcal{L}$-class has a unique idempotent, and so $M$ is a block group [12]. Conversely, it is shown in [24, 44] that, for a block group $M, S c h_{A}(X)$ is inverse for every regular $\mathcal{R}$-class $X$ and dually for $\mathcal{L}$-classes whence $M \in \mathbf{E J}$. The following is a key result of Henckell and Rhodes [24]; see 4] for a new proof, we shall give another later in this paper.

Theorem 7.7. $\mathbf{J} * \mathbf{G}=\mathbf{J}(m) \mathbf{G}$.

The above theorem and the following result will be used shortly to show that, for extension-closed pseudovarieties of groups, $\mathbf{J} * \mathbf{H}=\mathbf{J} m \mathbf{H}$ as well as various generalizations.

Theorem 7.8. Let $\mathbf{H}$ be an extension-closed pseudovariety of groups. Let $M \in$ $\mathbf{R}(m) \mathbf{H}$ or $\mathbf{L} \rightarrow \mathbf{H}$ be a finite A-generated monoid. Let $m \in M$ and

$$
L_{m}=\left\{w \in A^{*} \mid[w]_{M}=m\right\} .
$$

Then $c l_{\mathbf{H}}\left(L_{m}\right)=\operatorname{cl}_{\mathbf{G}}\left(L_{m}\right)$ in $F G(A)$.

Proof. We just handle the case $M \in \mathbf{R} \rightarrow \mathbf{H}$. The language $L_{m}$ is rational, being recognized by $\left(\Gamma_{A}(M), 1\right)$. But, by Theorem $7.4,\left(\Gamma_{A}(M), 1\right)$ is locally $\mathbf{H}$-extendible. So, by Theorem 6.31 the result follows.

We now point out that the membership problems for the different pseudovarieties we have been considering are equivalent.

Theorem 7.9. Let $\mathbf{H}$ be a pseudovariety of groups. Then the membership problems for $\mathbf{R} \rightarrow \mathbf{H}, \mathbf{L} \rightarrow \mathbf{H}, \mathbf{J} \rightarrow \mathbf{H}$, and the pseudovariety of inverse monoids $\mathbf{S l} \rightarrow \mathbf{H}$ are equivalent.

Proof. By Theorem 7.4 if the membership problem for $\mathbf{S l} m \mathbf{H}$ as a pseudovariety of inverse monoids is decidable, then the membership problems for $\mathbf{R} m \mathbf{H}, \mathbf{L} m \mathbf{H}$, and $\mathbf{J} \rightarrow \mathbf{H}$ are all decidable. Since a monoid $M \in \mathbf{R} m \mathbf{H}$ if and only if $M^{\rho} \in \mathbf{L} m \mathbf{H}$, these pseudovarieties have equivalent membership problems. Hence if $\mathbf{R} m \mathbf{H}$ is decidable, so is $\mathbf{J}: \mathbf{H}=(\mathbf{R}: \mathbf{H}) \cap(\mathbf{L}(m) \mathbf{H})$. Now suppose $\mathbf{J}: \mathbf{H}$ has decidable membership problem. Let $I$ be a finite inverse monoid. Then if $I \in \mathbf{S l} m \mathbf{H}, I \in \mathbf{J} m \mathbf{H}$. Conversely, if $K_{\mathbf{H}}(I) \in \mathbf{J}$, then, since $K_{\mathbf{H}}(I)$ is a subinverse monoid and the only $\mathcal{J}$-trivial inverse monoids are semilattices, $K_{\mathbf{H}}(I) \in \mathbf{S l}$, and so $I \in \mathbf{S l}: \mathbf{H}$. Thus if $\mathbf{J}: \mathbf{H}$ has a decidable membership problem, so does the pseudovariety of inverse monoids $\mathbf{S l}+\mathbf{H}$. 
Corollary 7.10. Let $\mathbf{H}$ be a pseudovariety of groups such that there is an algorithm, given a finite set of reduced words $Y$ over a finite set $A$, to decide membership in $\operatorname{cl}_{\mathbf{H}}(\langle Y\rangle) \subseteq F G(A)$. Then $\mathbf{R}: \mathbf{H}, \mathbf{L} \rightarrow \mathbf{H}$, and $\mathbf{J} \rightarrow \mathbf{H}$ all have decidable membership problem.

Such algorithms exists for $\mathbf{G}_{p}[30,38], \mathbf{G}_{n i l}[30$, and any decidable pseudovariety of abelian groups [42].

7.2. Regular Elements of $K_{\mathbf{H}}$. We now show that the membership problem for the pseudovariety of inverse monoids $\mathbf{S l} \mathrm{m} \mathbf{H}$ is equivalent to the computability of the regular elements of $K_{\mathbf{H}}(M)$ given $M$ as input. This was proved by the author in 44] using profinite monoids, but our proof here will be more geometric in nature. First we show that $K_{\mathbf{H}}$ is a functor.

Proposition 7.11. Let $\mathbf{H}$ be a pseudovariety of groups. Then $K_{\mathbf{H}}$ is a functor preserving surjective map.

Proof. Let $\varphi: M \rightarrow N$ be a morphism. If $m \in K_{\mathbf{H}}(M)$ and $\tau: N \bullet G \in \mathbf{H}$, then $1 \in m(\varphi \tau)=(m \varphi) \tau ;$ so $m \varphi \in K_{\mathbf{H}}(N)$. Suppose $\varphi$ is surjective. Let $\mu: M \mapsto G \in$ $\mathbf{H}$ be a relational morphism such that $m \in K_{\mathbf{H}}(M)$ if and only if $m \in 1 \mu^{-1}$. Then if $n \in K_{\mathbf{H}}(N), 1 \in n \varphi^{-1} \mu$, so there exists $m \in n \varphi^{-1}$ such that $1 \in m \mu$. But then $m \in K_{\mathbf{H}}(M)$. The result follows.

The following proposition is straightforward to verify. For a monoid $M$, we use $\operatorname{Reg}(M)$ to denote the set of regular elements of $M$. We want to show that $\operatorname{Reg}$ is a functor.

Lemma 7.12. Let $M$ be a finite monoid and $r, m \in M$. Then $(m r)^{\omega} m$ is regular with inverse $r(m r)^{\omega-1}$. Furthermore, $m \in \operatorname{Reg}(M)$ if and only if $m=(m r)^{\omega} m$ for some $r \in M$.

Proposition 7.13. Reg is a functor preserving surjective map.

Proof. Let $\varphi: M \rightarrow N$ be a morphism. Then the image of a regular element is clearly regular. Suppose $n \in \operatorname{Reg}(N)$ and $\varphi$ is onto. Let $s \in N$ such that $n=(n s)^{\omega} n$. Let $n=m \varphi, s=r \varphi$ for $m, r \in M$. Then $(m r)^{\omega} m \in \operatorname{reg}(M)$ and $\left((m r)^{\omega} m\right) \varphi=n$.

Proposition 7.14. Let $\mathbf{H}$ be a pseudovariety of groups. Then for $M$ a finite monoid, one has that $K_{\mathbf{H}}(M) \cap \operatorname{Reg}(M)=\operatorname{Reg}\left(K_{\mathbf{H}}(M)\right)$.

Proof. Clearly $\operatorname{Reg}\left(K_{\mathbf{H}}(M)\right) \subseteq K_{\mathbf{H}}(M) \cap \operatorname{Reg}(M)$. Suppose $m \in K_{\mathbf{H}}(M) \cap$ $\operatorname{Reg}(M)$. Let $r \in M$ be such that $m=(m r)^{\omega} m$. Let $\tau: M \multimap G \in \mathbf{H}$ be a relational morphism. Suppose $r \tau g$. Then $r(m r)^{\omega-1} \tau g g^{-1}=1$. Thus $r(m r)^{\omega-1} \in K_{\mathbf{H}}(M)$. But $m$ and $r(m r)^{\omega-1}$ are inverses, so $m \in \operatorname{Reg}\left(K_{\mathbf{H}}(M)\right)$.

We now relate the rational language associated to a regular element of $M$ to the corresponding language recognized by its Schützenberger graph.

Proposition 7.15. Let $M$ be a finite A-generated monoid, $m \in \operatorname{Reg}(M)$,

$$
L_{m}=\left\{u \in A^{*} \mid[u]_{M}=m\right\},
$$

$e \in R_{m}$ an idempotent, $\Gamma=\operatorname{Sch}_{A}\left(R_{m}\right)$, and

$$
L_{e, m}=\left\{u \in A^{*} \mid e u=m \text { in } \Gamma\right\} \text {. }
$$


Analogously, let

$$
L_{m}^{\prime}=\left\{\underline{w} \in F^{\omega-1}(A) \mid[\underline{w}]_{M}=m\right\}
$$

and

$$
L_{e, m}^{\prime}=\left\{\underline{w} \in F^{\omega-1}(A) \mid e \underline{w}=m \text { in } \Gamma\right\} .
$$

Let $\rho: F^{\omega-1}(A) \rightarrow F G(A)$ be the canonical surjection. Then

$$
c l_{\mathbf{G}}\left(L_{m}\right)=c l_{\mathbf{G}}\left(L_{e, m}\right)=L_{e, m}^{\prime} \rho=L_{m}^{\prime} \rho=\pi_{1}(\mathcal{I}(\Gamma),[e]) w
$$

where $w$ is any reduced word taking $[e]$ to $[m]$ in $\mathcal{I}(\Gamma)$.

Proof. First note that, since $e m=m, L_{m} \subseteq L_{e, m}$ and $L_{m}^{\prime} \subseteq L_{e, m}^{\prime}$. So we just need to show that $c l_{\mathbf{G}}\left(L_{e, m}\right) \subseteq c l_{\mathbf{G}}\left(L_{m}\right)$ and that $L_{e, m}^{\prime} \rho \subseteq L_{m}^{\prime} \rho$ since, by Theorem 6.11

$$
c_{\mathbf{G}}\left(L_{e, m}\right)=L_{e, m}^{\prime} \rho=\pi_{1}(\mathcal{I}(\Gamma),[e]) w .
$$

Let $u \in A^{*}$ such that $[u]_{M}=m$. Suppose $v \in c l_{\mathbf{G}}\left(L_{e, m}\right)$, then $u^{n !} v \in L_{m}$ for all $n$ and, since $u^{n !} \rightarrow 1$ in $F G(A)$, we see $v \in c l_{\mathbf{G}}\left(L_{m}\right)$. Similarly, if $\underline{w} \in L_{e, m}^{\prime}$, then $u^{\omega} \underline{w} \in L_{m}^{\prime}$ and $[\underline{w}]_{F G(A)}=\left[u^{\omega} \underline{w}\right]_{F G(A)}$. The result follows.

Note that, in Proposition 7.15 we could again replace $L_{m}^{\prime}$ with its subset consisting of terms of height at most one.

We draw several corollaries; this first one also uses Corollary 6.12 and Proposition 6.16

Corollary 7.16. Let $M$ be a finite A-generated monoid, $m \in \operatorname{Reg}(M)$,

$$
L_{m}=\left\{u \in A^{*} \mid[u]_{M}=m\right\},
$$

$e \in R_{m}$ an idempotent, $\Gamma=\operatorname{Sch}_{A}\left(R_{m}\right)$,

$$
L_{e, m}=\left\{u \in A^{*} \mid e u=m \text { in } \Gamma\right\},
$$

and $\mathbf{H}$ a pseudovariety of groups. Then

$$
c l_{\mathbf{H}}\left(L_{m}\right)=c l_{\mathbf{H}}\left(L_{e, m}\right)=c l_{\mathbf{H}}\left(\pi_{1}(\mathcal{I}(\Gamma),[e])\right) w=c l_{\mathbf{H}}\left(\pi_{1}\left(\mathcal{I}_{\mathbf{H}}(\Gamma),[e]_{\mathbf{H}}\right)\right) v
$$

where $w$ is any reduced word taking $[e]$ to $[m]$ in $\mathcal{I}(\Gamma)$ and $v$ is any reduced word taking $[e]_{\mathbf{H}}$ to $[m]_{\mathbf{H}}$ in $\mathcal{I}_{\mathbf{H}}(\Gamma)$.

The following result was first proved by Tilson in 48 in the case when $\mathbf{H}=\mathbf{G}$ and by the author in general [44.

Corollary 7.17. Let $M$ be a finite A-generated monoid, $m \in \operatorname{Reg}(M)$, and $e \in R_{m}$ be an idempotent. Then $m \in K_{\mathbf{H}}(M)$ if and only if $[e]_{\mathbf{H}}=[m]_{\mathbf{H}}$ in $\mathcal{I}_{\mathbf{H}}\left(\operatorname{Sch}_{A}(X)\right)$.

Proof. Indeed, $e \sim_{\mathbf{H}} m$ if and only if $1 \in c l_{\mathbf{H}}\left(L_{e, m}\right)=c l_{\mathbf{H}}\left(L_{m}\right)$, if and only if $m \in K_{\mathbf{H}}(M)$.

Corollary 7.18. The membership problems for the pseudovariety of inverse monoids $\mathbf{S l} m \mathbf{H}$ and the pseudovarieties of monoids $\mathbf{R} m \mathbf{H}, \mathbf{L} m \mathbf{H}$, and $\mathbf{J} m \mathbf{H}$ are all equivalent to the computability of the functor Reg $\circ K_{\mathbf{H}}$.

Proof. Suppose the pseudovariety of inverse monoids $\mathbf{S l} m \mathbf{H}$ has decidable membership problem. Then the above corollary shows that $R e g \circ K_{\mathbf{H}}$ is computable. Conversely, for an inverse monoid $I, K_{\mathbf{H}}(I)=\operatorname{Reg}\left(K_{\mathbf{H}}(I)\right)$. The result follows. 
The computability of $R e g \circ K_{\mathbf{H}}$, of course, implies the decidability of the membership problem for any Mal'cev product of the form RV $m \mathbf{H}$ where $\mathbf{R V}$ is the pseudovariety of monoids whose regular elements generate a monoid in V. For instance, the pseudovariety $\mathbf{A}=\llbracket x^{\omega-1}=x^{\omega} \rrbracket$ of all finite aperiodic monoids has the property that $\mathbf{R A}=\mathbf{A}$ and is local, so if $\operatorname{Reg} \circ K_{\mathbf{H}}$ is computable, then membership in $\mathbf{A} * \mathbf{H}$ is decidable.

7.3. An Application to Language Theory. We now give an application to language theory. If $\mathbf{H}$ is a pseudovariety of groups and $A$ a finite set, the pro-H topology on $A^{*}$ is defined to be the weakest topology such that every homomorphism $\varphi: A^{*} \rightarrow G \in \mathbf{H}$ is continuous where $G$ is given the discrete topology. Noting that every finite group is generated by $A$ as a group if and only if it is generated by $A$ as a monoid, it is easy to show that the pro-H topology on $A^{*} \subseteq F G(A)$ is the induced topology.

A language $L \subseteq A^{*}$ is said to be recognized by a monoid $M$ if there is a morphism $\varphi: A^{*} \rightarrow M$ and a subset $F \subseteq M$ such that $L=F \varphi^{-1}$. Equivalently, one can view $M$ as a, possibly disconnected, deterministic $A$-graph via $\varphi$ (as in the Cayley graph construction) and then $L$ is recognized by the connected component of 1 with final states those elements of $F$ in that component. Conversely, any rational language is recognized by the transition monoid of the corresponding automaton. Given a rational language $L$, there is a smallest monoid $M$ recognizing it, called the syntactic monoid of $L$ [14]. This monoid is given by the congruence $w \equiv v$ if, for all $x, y \in A^{*}, x w y \in L \Longleftrightarrow x v y \in L$.

Theorem 7.19. Let $\mathbf{H}$ be a pseudovariety of groups residually containing $F G(A)$ and $L \subseteq A^{*}$ a rational language which is open or closed in the pro- $\mathbf{H}$ topology. Then the syntactic monoid of $L$ is in $\mathbf{J}: \mathbf{H}$.

Proof. Since the syntactic monoid of a language $L$ and of its complement are the same, it suffices to assume $L$ is closed. Let $M$ be the syntactic monoid of $L$. By Theorem 7.4 it suffices to show that every left and right Schützenberger graph of $M$ is $\mathbf{H}$-extendible. To do this, first suppose $s \mathcal{R} t$ and that $s \sim_{\mathbf{H}} t$. Then $1 \in \operatorname{cl}_{\mathbf{H}}\left(L_{s, t}\right)$. Since, by assumption on $\mathbf{H}$, the pro-H topology is metric, there exists a sequence of words $\left\{w_{n}\right\} \in L_{s, t}$ converging to 1 . Let $w_{s}, w_{t} \in A^{*}$ be such that $\left[w_{s}\right]=s$ and $\left[w_{t}\right]=t$. Then $w_{s} w_{n} \equiv w_{t}$ for all $n$. So suppose $x, y \in A^{*}$ and $x w_{t} y \in L$. Then, since $w_{t} \equiv w_{s} w_{n}$ for all $n, x w_{s} w_{n} y \in L$ for all $n$ whence, since $L$ is closed, $x w_{s} y \in L$ by continuity of multiplication. Since $1 \in c l_{\mathbf{H}}\left(L_{t, s}\right)$ as well, we see that the reverse implication also holds; thus $s=t$. We conclude that the Schützenberger graph for every $\mathcal{R}$-class is $\mathbf{H}$-extendible. A dual argument holds for $\mathcal{L}$-classes. The result follows.

7.4. The Main Theorem. In this subsection, we prove that the equality $\mathbf{J} m \mathbf{H}=$ $\mathbf{J} * \mathbf{H}$ holds for extension-closed pseudovarieties of groups. To do this, we need the following two definitions due to Henckell and Rhodes. If $X$ is a subset of a finite monoid $M$ and $\mathbf{V}$ is a pseudovariety of monoids, $X$ is said to be $\mathbf{V}$-pointlike if, for every relational morphism $\mu: M \hookrightarrow V \in \mathbf{V}$, there exists $v \in V$ such that $X \subseteq v \mu^{-1}$. One says that $\mathbf{V}$ has decidable pointlikes if one can decide, given as input a finite monoid and a subset of that monoid, whether that subset is $\mathbf{V}$ pointlike. For example, if $\mathbf{H}$ is a pseudovariety of groups, then $K_{\mathbf{H}}(M)$ is a pointlike subset of $M$ (and is, in fact, the maximal pointlike subset $X$ such that $X^{2}=X$ ). If $M$ is a finite monoid and $\mathbf{H}$ a pseudovariety of groups, $\left(m_{1}, \ldots, m_{k}\right) \in M^{k}$ is 
called an $\mathbf{H}$-liftable $k$-tuple if, for every relational morphism $\mu: M \multimap G \in \mathbf{H}$, there exist $g_{1}, \ldots, g_{k} \in G$ such that $g_{1} \cdots g_{k}=1$ and $g_{i} \in m_{i} \mu$ for all $i$. Note that $(m)$ is an $\mathbf{H}$-liftable 1-tuple if and only if $m \in K_{\mathbf{H}}(M)$. The proof of the following proposition is similar to that of Proposition [5.7; see for instance [38].

Proposition 7.20. Let $M$ be a finite A-generated monoid, $\left(m_{1}, \ldots, m_{k}\right) \in M^{k}$,

$$
L_{m_{i}}=\left\{u \in A^{*} \mid[u]_{M}=m_{i}\right\},
$$

and $\mathbf{H}$ be a pseudovariety of groups. Then $\left(m_{1}, \ldots, m_{k}\right)$ is an $\mathbf{H}$-liftable $k$-tuple if and only if $1 \in c l_{\mathbf{H}}\left(L_{m_{1}} \cdots L_{m_{k}}\right)$.

Our results on languages recognized by locally $\mathbf{H}$-extendible automata imply the following.

Theorem 7.21. Let $\mathbf{H}$ be an extension-closed pseudovariety of groups and $M \in$ $\mathbf{R}\left(m \mathbf{H}\right.$ or $\mathbf{L}\left(m \mathbf{H}\right.$. Then $\left(m_{1}, \cdots, m_{k}\right) \in M^{k}$ is an $\mathbf{H}$-liftable $k$-tuple if and only if it is a $\mathbf{G}$-liftable $k$-tuple

Proof. We suppose $M \in \mathbf{R}(m \mathbf{H}$, the other case being dual. Let $A$ be a generating set for $M$. Then, by Theorem [7.8, for any $m \in M, c l_{\mathbf{H}}\left(L_{m}\right)=c l_{\mathbf{G}}\left(L_{m}\right)$. Now, by Corollary 6.25,

$$
\begin{aligned}
& c l_{\mathbf{H}}\left(L_{m_{1}} \cdots L_{m_{k}}\right)=c l_{\mathbf{H}}\left(L_{m_{1}}\right) \cdots c l_{\mathbf{H}}\left(L_{m_{k}}\right) \\
& \quad=c l_{\mathbf{G}}\left(L_{m_{1}}\right) \cdots c l_{\mathbf{G}}\left(L_{m_{k}}\right)=c l_{\mathbf{G}}\left(L_{m_{1}} \cdots L_{m_{k}}\right) .
\end{aligned}
$$

The result now follows from Proposition 7.20

We now recall [44, Theorem 8.6].

Theorem 7.22. Let $M$ be a block group and $\mathbf{H}$ a pseudovariety of groups. Then $M \in \mathbf{J} * \mathbf{H}$ if and only if, for every pair $\{a, c\}$ of regular elements of $M$ which is $\mathbf{H}-$ pointlike,

$$
a a^{-1} c c^{-1}=a c^{-1} \text {. }
$$

The reader should recall that in a block group, each regular element has a well defined inverse and that $\mathbf{J} m \mathbf{H} \subseteq \mathbf{B G}$. We wish to turn the above condition into a condition on $\mathbf{H}$-liftable tuples.

Lemma 7.23. Let $\mathbf{H}$ be a pseudovariety of groups, $M$ a block group, and a, $c \in$ $\operatorname{Reg}(M)$. Then $\{a, c\}$ is $\mathbf{H}$-pointlike if and only if $\left(a, c^{-1}\right)$ is an $\mathbf{H}$-liftable 2-tuple.

Proof. Suppose $\mu: M \multimap G \in \mathbf{H}$ is a relational morphism. Then if $c \mu h$ and $c^{-1} \mu g$,

$$
c=\left(c c^{-1}\right)^{\omega-1} c \mu(h g)^{-1} h=g^{-1}
$$

and, similarly, $c^{-1} \mu h^{-1}$. So if $\{a, c\} \subseteq h \mu^{-1}$, then $a \mu h, c^{-1} \mu h^{-1}$, and $h h^{-1}=1$. Similarly, if $a \mu g, c^{-1} \mu g^{-1}$, then $c \mu g$, and so $\{a, c\}$ is $\mathbf{H}$-pointlike if and only if $\left(a, c^{-1}\right)$ is an $\mathbf{H}$-liftable 2-tuple.

Theorem 7.24. Suppose $\mathbf{H}$ is an extension-closed pseudovariety of groups. Then $\mathbf{J}[\mathbf{H}=\mathbf{J} * \mathbf{H}$.

Proof. Let $M \in \mathbf{J}(m \mathbf{H}$ and suppose $a, c \in \operatorname{Reg}(m)$ are such that $\{a, c\}$ is $\mathbf{H}$ pointlike. Then, by Lemma 7.23, $\left(a, c^{-1}\right)$ is an $\mathbf{H}$-liftable 2-tuple. So, by Theorem[7.21 $\left(a, c^{-1}\right)$ is also a $\mathbf{G}$-liftable 2-tuple, and hence $\{a, c\}$ is $\mathbf{G}$-pointlike. Now, since $M \in \mathbf{J}: \mathbf{G}=\mathbf{J} * \mathbf{G}$, by Theorem [7.22, $a a^{-1} c c^{-1}=a c^{-1}$. Another application of Theorem 7.22 then shows that $M \in \mathbf{J} * \mathbf{H}$. 
Of course, this short proof is one of the aims of this paper. But after building all this machinery, it might be difficult for the reader to see what is truly happening. Also, this proof may be unsatisfactory as it requires the result of Henckell and Rhodes, both known proofs of which are long [4 24]. But again, our techniques come to the rescue. We now give an independent proof of the above theorem which, for the case $\mathbf{H}=\mathbf{G}$, is considerably shorter than either of the existing proofs. Also this proof does not use Ash's Theorem, but rather the simpler to prove cases $n=1,2$ of the Ribes and Zalesskiı Theorem [20, 33].

Using Lemma 7.23 and Theorem 7.22 it suffices to prove that if $M \in \mathbf{J}: \mathbf{H}$ and $(a, c)$ is an $\mathbf{H}$-liftable 2-tuple with $a, c \in \operatorname{Reg}(M)$, then

$$
a a^{-1} c^{-1} c=a c .
$$

So suppose $(a, c)$ is such an $\mathbf{H}$-liftable 2-tuple and $M$ is $A$-generated. Let

$$
L_{a}=\left\{u \in A^{*} \mid[u]_{M}=a\right\}
$$

and $L_{c}$ be the analogous language for $c$. Let $\Gamma_{a}=S c h_{A}(X)$ and $\Gamma_{c}=S c h_{A}^{\rho}(Y)$ where $X$ is the $\mathcal{R}$-class of $a$ and $Y$ is the $\mathcal{L}$-class of $c$. Recall that, since $M$ is a block group, $\Gamma_{a}$ and $\Gamma_{c}$ are inverse $A$-graphs.

Lemma 7.25. Let $w \in \tilde{A}^{*}$ such that $a a^{-1} w=b$ in $\Gamma_{a}$ and $w^{-1} c^{-1} c=d$ in $\Gamma_{c}$. Then

$$
a a^{-1}(b d) c^{-1} c=a a^{-1} c^{-1} c .
$$

Proof. The proof is by induction. If $|w|=0, b=a a^{-1}, d=c^{-1} c$, and $a a^{-1} b d c^{-1} c=$ $a a^{-1} c^{-1} c$. Suppose $w=v x^{-1}$ with $x \in A$, the other case being dual. Let $b \stackrel{x}{\longrightarrow} b^{\prime}$ be the positively oriented edge whose reverse is the last edge used in the run of $w$ from $a a^{-1}$ and $d=x d^{\prime}\left(d^{\prime}\right.$ is well defined since $w^{-1}$ has a reverse run from $c^{-1} c$ to $d$ and the graph is inverse). Then

$$
a a^{-1}(b d) c^{-1} c=a a^{-1} b x d^{\prime} c^{-1} c=a a^{-1}\left(b^{\prime} d^{\prime}\right) c^{-1} c .
$$

But $a a^{-1} v=b^{\prime}$ in $\Gamma_{a}$ and $v^{-1} c^{-1} c=d^{\prime}$ in $\Gamma_{c}$, so, by induction, the right-hand side of the above equation is equal to $a a^{-1} c^{-1} c$.

Let $H=\pi_{1}\left(\Gamma_{a}, a a^{-1}\right), K=\pi_{1}\left(\Gamma_{c}, c^{-1} c\right)^{\rho}$, and $u, v \in A^{*}$ be such that $[u]_{M}=a$, $[v]_{M}=c$. Since $\Gamma_{a}$ and $\Gamma_{c}$ are $\mathbf{H}$-extendible and, furthermore, $\mathbf{H}$ is extensionclosed, $H$ and $K$ are $\mathbf{H}$-closed, finitely generated subgroups. We then have

$$
c l_{\mathbf{H}}\left(L_{a}\right)=H u, c l_{\mathbf{H}}\left(L_{c}\right)=v K,
$$

and

$$
\begin{gathered}
c l_{\mathbf{H}}\left(L_{a} L_{c}\right)=c l_{\mathbf{H}}(H u v K)=u v c l_{\mathbf{H}}\left((u v)^{-1} H u v K\right) \\
=u v c l_{\mathbf{H}}\left((u v)^{-1} H u v\right) c l_{\mathbf{H}}(K)=H u v K=c l_{\mathbf{H}}\left(L_{a}\right) c l_{\mathbf{H}}\left(L_{c}\right)
\end{gathered}
$$

by Theorem 5.19, Since $(a, c)$ is a liftable $\mathbf{H}$-tuple, $1 \in c l_{\mathbf{H}}\left(L_{a} L_{c}\right)=H u v K$, and so there exists $w \in \tilde{A}^{*}$ such that $w \in H u$ and $w^{-1} \in v K$. But then $a a^{-1} w=a$ in $\Gamma_{a}$ and $w^{-1} c^{-1} c=c$ in $\Gamma_{c}$. Hence, by Lemma [7.25,

$$
a c=a a^{-1}(a c) c^{-1} c=a a^{-1} c^{-1} c .
$$

Theorem 7.24 follows. 
Lemma 7.26. Let $\mathbf{V}$ and $\mathbf{W}$ be pseudovarieties of monoids and $\mathbf{H}$ a pseudovariety of groups. Then

$$
(\mathbf{V} \cap \mathbf{W})(m \mathbf{H}=(\mathbf{V}: \mathbf{H}) \cap(\mathbf{W}[\mathbf{H}) .
$$

Proof. Both pseudovarieties consist of those monoids $M$ with $K_{\mathbf{H}}(M) \in \mathbf{V} \cap \mathbf{W}$.

We then get the following corollary of Theorem 7.21

Corollary 7.27. Let $\mathbf{H}$ be an extension-closed pseudovariety of groups and $\mathbf{V}$ a pseudovariety of monoids. Then

$$
(\mathbf{V} \cap \mathbf{R})(m \mathbf{H}=(\mathbf{V}: \mathbf{G}) \cap(\mathbf{R}: \mathbf{H}) .
$$

A dual result holds for $\mathbf{L}$.

Proof. For $M \in \mathbf{R}\left(m \mathbf{H}, K_{\mathbf{H}}(M)=K_{\mathbf{G}}(M)\right.$ by Theorem 7.21 The result then follows from the above lemma.

Recalling that $\mathbf{R}: \mathbf{H}=\mathbf{R} * \mathbf{H}$, we get the following corollary.

Corollary 7.28. Let $\mathbf{H}$ be an extension-closed pseudovariety of groups and $\mathbf{V} a$ local pseudovariety of monoids. Then

$$
(\mathbf{V} * \mathbf{H}) \cap(\mathbf{R} * \mathbf{H})=(\mathbf{V} * \mathbf{G}) \cap(\mathbf{R} * \mathbf{H}) .
$$

A dual result holds for $\mathbf{L}$.

Let Com be the pseudovariety of commutative monoids. Note that ECom = ESl.

Corollary 7.29. Let $\mathbf{H}$ be an extension-closed pseudovariety of groups. Then

$$
\mathbf{E C o m} \cap(\mathbf{R} * \mathbf{H})=\mathbf{S l} * \mathbf{H}=\mathbf{E C o m} \cap(\mathbf{L} * \mathbf{H}) .
$$

Proof. By a result of Ash [9, $\mathbf{E C o m}=\mathbf{S l} * \mathbf{G}$. It is well known that $\mathbf{S l}$ is local. The result then follows from the above corollary upon noting that $\mathbf{S l} * \mathbf{H} \subseteq(\mathbf{R} *$ $\mathbf{H}) \cap(\mathbf{L} * \mathbf{H})$.

In the next section, we shall show that every equality proved in this section can fail if $\mathbf{H}$ is not extension-closed. In fact, we shall obtain classes of pseudovarieties $\mathbf{H}$ for which these equations fail, although we do not yet have a complete classification.

7.5. Examples and Counterexamples. We begin by showing that, for pseudovarieties $\mathbf{H}$ of groups which are not extension-closed, $\mathbf{S l} * \mathbf{H}$ need not equal $\mathbf{E C o m} \cap(\mathbf{R} * \mathbf{H})$. In fact, our examples will be in $\mathbf{J} \cap \mathbf{E C o m}$. In this section, we shall often abuse notation by not distinguishing between generators and their images in a monoid.

Theorem 7.30. Let $\mathbf{H}$ be a pseudovariety of groups satisfying $x y=y x$ or an identity of the form $x^{n}=1$ for some $n>0$. Then there exists a monoid in $\mathbf{J} \cap \mathbf{E C o m}$ which is not in $\mathbf{S l} * \mathbf{H}$, and so $\mathbf{E C o m} \cap(\mathbf{R} * \mathbf{H}) \neq \mathbf{S l} * \mathbf{H}$.

Proof. Suppose first that $\mathbf{H}$ satisfies $x^{n}=1$ for $n>0$. Let

$$
M=\left\langle x \mid x^{n+1}=0\right\rangle .
$$

Clearly, $x^{n} \in K_{\mathbf{H}}(M)$; but $x^{n}$ is not an idempotent whence $M \notin \mathbf{S l} * \mathbf{H}$. However, $M \in \mathbf{J} \cap \mathbf{E C o m}$. On the other hand, the syntactic monoid of the language $a b^{*} a b a^{*} b$ over the alphabet $\{a, b\}$ is known not to be in $\mathbf{S l} * \mathbf{G}_{c o m}$ [27]. But a calculation with "AMoRE" [31], shows that this monoid is in $\mathbf{J} \cap \mathbf{E C o m}$. Hence equality fails for any pseudovariety $\mathbf{H}$ satisfying $x y=y x$. 
We now prove the more difficult result that $\mathbf{J} m \mathbf{H} \neq \mathbf{J} * \mathbf{H}$ for the same classes of pseudovarieties.

Theorem 7.31. Let $\mathbf{H}$ be a non-trivial pseudovariety of groups satisfying the identity $x y=y x$. Then $\mathbf{J}: \mathbf{H} \neq \mathbf{J} * \mathbf{H}$.

Proof. We shall exhibit a monoid which is in $\mathbf{J}: \mathbf{H}$ for every non-trivial pseudovariety of groups $\mathbf{H}$, but which is not in $\mathbf{J} * \mathbf{G}_{\text {com }}$. The result will then follow. Consider first the following inverse automaton $\left(\Gamma, v_{0}\right)$ over the alphabet $\{a, b\}$.

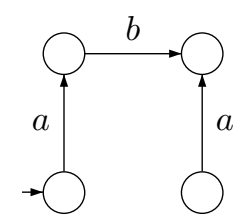

Let $I=M(\mathcal{I}(\Gamma))$. Using "AMoRE", we obtain the following monoid presentation,

$$
\begin{gathered}
I=\langle a, b, c, d| a^{2}=b a=b^{2}=c b=c^{2}=c d=d a=d^{2}=0, \\
a c a=a, b c a=b, b d b=b, c a b=b, c a c=c, c a d=d, d b d=d, d c a=d\rangle .
\end{gathered}
$$

This monoid has 22 elements and the following $\mathcal{J}$-class structure.

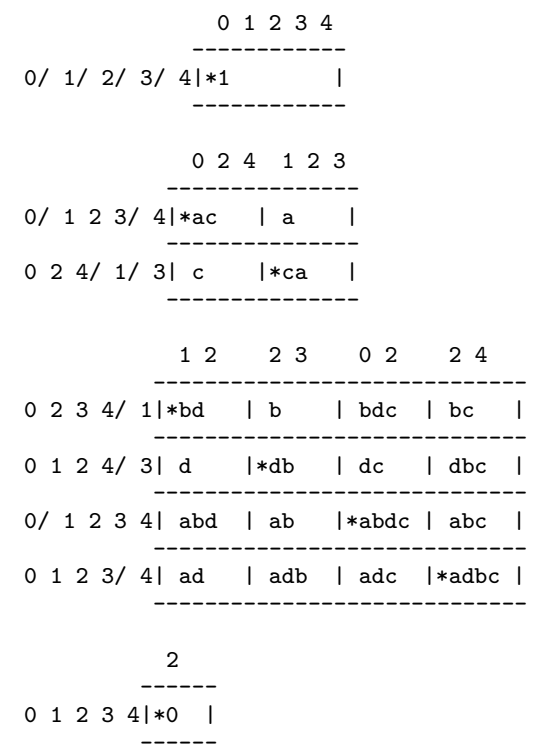

Suppose now that $\mathbf{H}$ is non-trivial. Then it contains a cyclic group $\mathbb{Z} / n \mathbb{Z}$ with $n>1$. Clearly, $\Gamma$ embeds in the Cayley graph of $\mathbb{Z} / n \mathbb{Z} \times \mathbb{Z} / n \mathbb{Z}$, so $\left(\Gamma, v_{0}\right)$ is $\mathbf{H}$-extendible and thus $I \in \mathbf{S l} \rightarrow \mathbf{H}$. Let

$$
\begin{gathered}
M=\langle a, b, c, d| a^{2}=b a=b^{2}=c b=c^{2}=d a=d^{2}=c d c=d c d=c d b c=0, \\
a c a=a, c a c=c, d b d=d, b d b=b, c a b=b c a, d c a=c a d, d b c d=c d\rangle .
\end{gathered}
$$

Using "AMoRE," one can verify that this monoid has 34 elements and the following $\mathcal{J}$-class structure. 


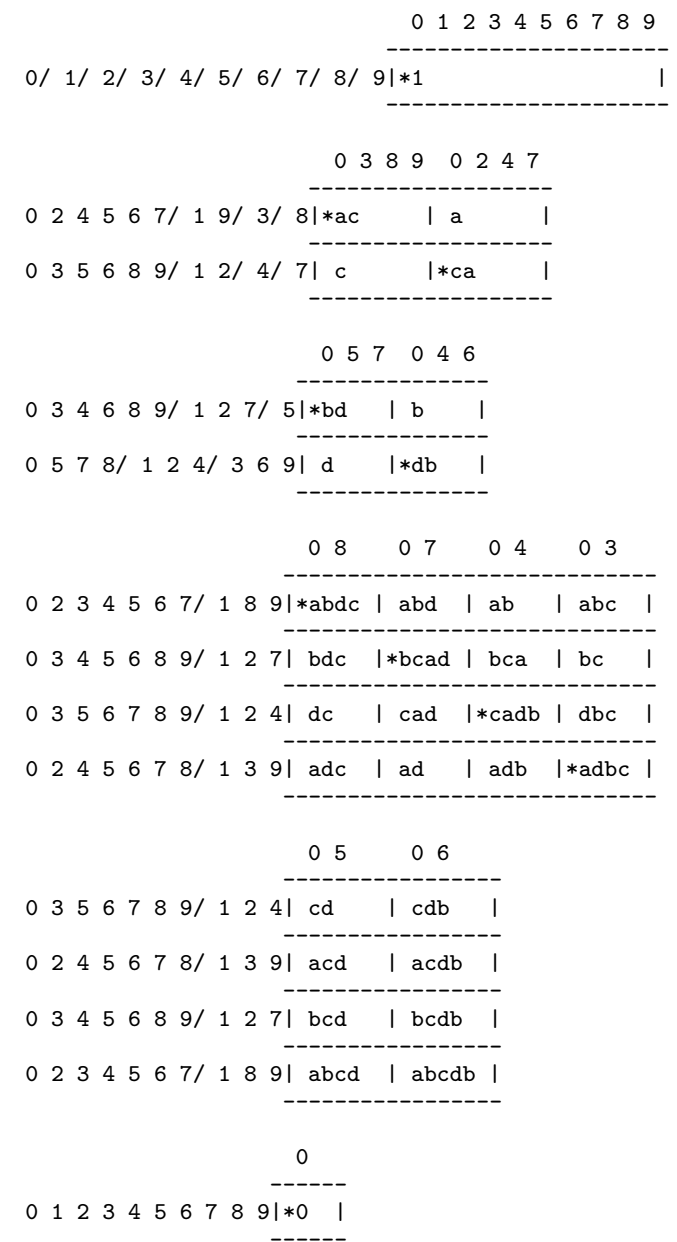

A simple verification shows that $I$ satisfies every relation $M$ does. Let $\varphi: M \rightarrow I$ be the natural homomorphism. Let

$$
N=0 \varphi^{-1}=\{c d, c d b, a c d, a c d b, b c d, b c d b, a b c d, a b c d b, 0\} .
$$

Then $N$ is a null ideal: $N^{2}=0$. Let $I^{\prime}=M / N$. Then $I^{\prime}$ is an inverse monoid and $\varphi$ factors as $\alpha \beta$ with $\alpha: M \rightarrow I^{\prime}$ and $\beta: I^{\prime} \rightarrow I$. It is easy to see that $(b c a) \beta=b$, $(c a d) \beta=d,(b c a d) \beta=b d,(c a d b) \beta=d b$, and $\beta$ sends every other element to itself; hence $\beta$ is idempotent pure, that is, $V_{\beta}=\mathbf{S l}$. Since $\beta: K_{\mathbf{H}}\left(I^{\prime}\right) \rightarrow K_{\mathbf{H}}(I)$, $K_{\mathbf{H}}(I)=E(I)$, and $\beta$ is idempotent pure, it follows that $K_{\mathbf{H}}\left(I^{\prime}\right)=E\left(I^{\prime}\right)$, and so $I^{\prime} \in \mathbf{S l}\left(m \mathbf{H}\right.$. Now, since $N^{2}=0$, the Mal'cev kernel of $\alpha$ satisfies the identity $x^{2}=0$. So $M \in \mathbf{N} m(\mathbf{S l} m \mathbf{H}) \subseteq \mathbf{J}(m) \mathbf{H}$.

We now show that $M \notin \mathbf{J} * \mathbf{G}_{c o m}$. Consider the pair of regular elements $\{a b c, b\}$. This set is $\mathbf{G}_{c o m}$-pointlike. Indeed, if $\mu: M \hookrightarrow G \in \mathbf{G}_{c o m}$ is a relational morphism and $a \varphi g$ and $b \varphi h$ then, as in the proof of Lemma 7.23 since $c=a^{-1}, c \varphi g^{-1}$. So $a b c \varphi g h g^{-1}=h$, since $G$ is abelian. Observing that $(a b c)^{-1}=a d c$ and $c b=0$, we see that

$$
a b c(a b c)^{-1} b b^{-1}=a b c a d c b d=0 .
$$


But $(a b c) b^{-1}=a b c d \neq 0$. Thus, by Theorem $7.22, M \notin \mathbf{J} * \mathbf{G}_{c o m}$.

Our next result is a bit more difficult to prove. A pseudovariety $\mathbf{V}$ is said to be locally finite if it has a largest monoid generated by any finite set. Zel'manov's solution of the restricted Burnside problem [51] shows that a pseudovariety of groups is locally finite if and only if it satisfies an identity of the form $x^{n}=1$ for some $n>0$. We now show that, for any non-trivial, locally finite pseudovariety of groups $\mathbf{H}, \mathbf{J}: \mathbf{H} \neq \mathbf{J} * \mathbf{H}$.

Theorem 7.32. Let $\mathbf{H}$ be a non-trivial pseudovariety of groups satisfying an identity of the form $x^{n}=1$ with $n>1$. Then $\mathbf{J}: \mathbf{H} \neq \mathbf{J} * \mathbf{H}$.

Proof. The idea of the proof is to construct a sequence of monoids with the property that if $\mathbf{H}$ satisfies $x^{n}=1$ with $n$ as small as possible (that is, $\mathbf{H}$ contains a cyclic group of order $n$, but none of larger order) then the $n^{t h}$ element of this sequence will be in $\mathbf{J} m \mathbf{H}$, but not in $\mathbf{J} * \mathbf{H}$. So let $n$ be the least positive integer such that $\mathbf{H}$ satisfies $x^{n}=1$. We consider the following inverse automaton $\left(\Gamma_{n}, v_{0}\right)$ over the alphabet $\{a\}$.

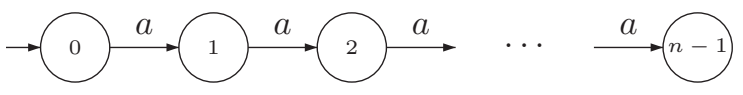

Let $I_{n}=M\left(\mathcal{I}\left(\Gamma_{n}, v_{0}\right)\right)$. It is shown in [6] that $I_{n}$ has the following monoid presentation:

$$
\begin{aligned}
& \langle a, b| a^{n}=b^{n}=0, b^{k} a^{k} b=b^{k} a^{k-1}, b a^{k} b^{k}=a^{k-1} b^{k}, \\
& \left.a^{k} b^{k} a=a^{k} b^{k-1}, a b^{k} a^{k}=b^{k-1} a^{k}(1 \leq k \leq n-1)\right\rangle .
\end{aligned}
$$

Furthermore, they show that each non-zero element can be put uniquely in the form $b^{i} a^{j} b^{k}$ with $0 \leq i, k \leq j<n$ (below, we shall give a proof that will imply that every non-zero element can be put in such a form, uniqueness follows from a computation with the above automaton).

We note that since $\left(\Gamma_{n}, v_{0}\right)$ clearly embeds in the Cayley graph of $\mathbb{Z} / n \mathbb{Z}$, it is $\mathbf{H}$-extendible by choice of $n$ whence $I_{n} \in \mathbf{S l} m \mathbf{H}$. Our desired monoid will be obtained from $I_{n}$ by replacing 0 with a null ideal. Let

$$
\begin{gathered}
M_{n}=\langle a, b| a^{n+1}=b^{n}=0, b^{k} a^{k} b=b^{k} a^{k-1}, b a^{k} b^{k}=a^{k-1} b^{k}, \\
\left.a^{k} b^{k} a=a^{k} b^{k-1}, a b^{k} a^{k}=b^{k-1} a^{k}(1 \leq k \leq n-1)\right\rangle
\end{gathered}
$$

and

$$
M_{n}^{\prime}=\left\langle a, b \mid a^{n+1}=b^{2}=0, b a b=b, a b a=a\right\rangle .
$$

Then it is easy to see that there are natural surjective homomorphisms $\varphi: M_{n} \rightarrow I_{n}$ and $\psi: M_{n} \rightarrow M_{n}^{\prime}$. We first show that $I_{n}=M_{n} / N$ where $N \subseteq M_{n}$ is an ideal such that $N^{2}=0$. Then we shall show that certain elements of $N$ are non-zero by making use of a representation of $M_{n}^{\prime}$ as the transition monoid of an automaton. We begin by showing that every non-zero element of $M_{n}$ can be put in the form $b^{i} a^{j} b^{k}$ with $0 \leq i, k \leq \min \{j, n-1\}, 0 \leq j \leq n$. The proof is by induction on $|u|$, $u \in\{a, b\}^{*}$. We use $\sim$ for the congruence on words associated to $M_{n}$. If $|u|=0$, then $u=b^{0} a^{0} b^{0}$ so we suppose $|u|>0$. 
Case 1: Suppose that $u=v a$. Then if $v \sim 0, u \sim 0$ and we are done. So we may suppose, by induction, that $v \sim b^{i} a^{j} b^{k}$ with $0 \leq i, k \leq \min \{j, n-1\}, 0 \leq j \leq n$. If $k>0$, then

$$
u \sim b^{i} a^{j} b^{k} a=b^{i} a^{j-k}\left(a^{k} b^{k} a\right) \sim b^{i} a^{j-k}\left(a^{k} b^{k-1}\right)=b^{i} a^{j} b^{k-1}
$$

which is of the desired form. If $k=0$, then $u \sim b^{i} a^{j} a=b^{i} a^{j+1}$ which is 0 if $j=n$, and is otherwise of the desired form.

Case 2: Suppose $u=v b$. Again, if $v \sim 0, u \sim 0$, we are done. Otherwise, by induction, we may assume that $v \sim b^{i} a^{j} b^{k}$ with $0 \leq i, k \leq \min \{j, n-1\}, 0 \leq j \leq n$. Then $u \sim b^{i} a^{j} b^{k+1}$. If $k<\min \{j, n-1\}$ we are done. If $k=n-1$, then $u \sim 0$ and we are also done. So we may suppose $k=j<n-1$. Then

$$
u \sim b^{i}\left(a^{j} b^{j+1}\right) \sim b^{i}\left(b a^{j+1} b^{j+1}\right)=b^{i+1} a^{j+1} b^{j+1}
$$

which is of the desired form.

Note that this proof shows that any non-zero element of $I_{n}$ can be placed in the aforementioned form. Also, it shows that any non-zero element of $M_{n}^{\prime}$ can be put in the form $b^{i} a^{j} b^{k}$ with $0 \leq i, k \leq \min \{1, j\}, 0 \leq j \leq n$. It now follows that $\varphi$ is injective when restricted to those elements which do not map to 0 . The remaining elements form an ideal

$$
N=0 \varphi^{-1}=0 \cup\left\{b^{i} a^{n} b^{k} \mid 0 \leq i, k \leq n-1\right\}
$$

such that $M_{n} / N=I_{n}$. Of course, we haven't shown that the elements of this ideal are distinct. We shall do this by first showing that $a^{n} \neq 0$, and then, as a consequence, prove that all these elements are distinct. To prove that $a^{n} \neq 0$, it will suffice to show that the normal forms above for $M_{n}^{\prime}$ represent distinct elements. First, however, we shall show that $N^{2}=0$. Indeed,

$$
b^{i_{1}} a^{n} b^{k_{1}} b^{i_{2}} a^{n} b^{k_{2}}=b^{i_{1}} a^{n} b^{k_{1}+i_{2}} a^{n} b^{k_{2}} .
$$

Let $r=k_{1}+i_{2}$. If $r \geq n$, then the right-hand side is 0 in $M_{n}$ as desired. If $r<n$,

$$
a^{n} b^{r} a^{n}=a^{n-r} a^{r} b^{r} a a^{n-1} \sim a^{n-r} a^{r} b^{r-1} a^{n-1}=a^{n} b^{r-1} a^{n-1} .
$$

Proceeding inductively, we see that $a^{n} b^{r} a^{n} \sim a^{n} a^{n-r} \sim 0$ since $r<n$. Thus $N^{2}=0$.

Now we show that the aforementioned elements give a set of normal forms for $M_{n}^{\prime}$. Consider the following automaton $\left(\Delta_{n}, 0\right)$ over $\{a, b\}$.

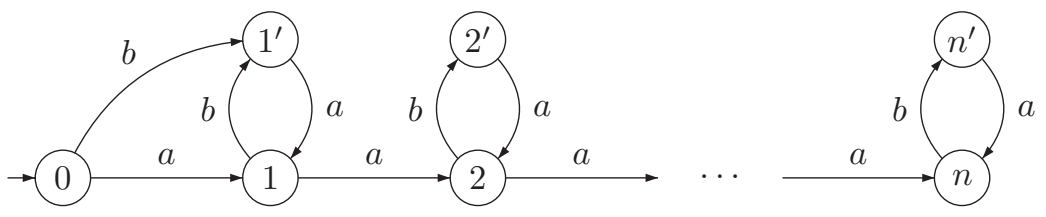

We show that $M\left(\Delta_{n}\right)=M_{n}^{\prime}$ and that our normal forms can be distinguished by this automaton.

It is easy to verify that $M\left(\Delta_{n}\right)$ satisfies the defining relations of $M_{n}^{\prime}$, so there is a natural surjection $\rho: M_{n}^{\prime} \rightarrow M\left(\Delta_{n}\right)$. Let $0 \leq i, k \leq \min \{1, j\}, 0 \leq j \leq n$. Then an easy calculation shows that $0 b=1^{\prime}, 1^{\prime} b$ is undefined, and, for $j>0$,

$$
0 b^{i} a^{j} b^{k}= \begin{cases}j & \text { if } k=0, \\ j^{\prime} & \text { if } k=1,\end{cases}
$$


and

$$
1^{\prime} b^{i} a^{j} b^{k}= \begin{cases}\text { undefined } & \text { if } i=1, \\ j & \text { if } i=0, k=0, \\ j^{\prime} & \text { if } i=0, k=1 .\end{cases}
$$

So evaluation at $1^{\prime}$ uniquely determines $i$, while evaluation at 0 uniquely determines $j$ and $k$. So it follows that the normal forms give distinct elements and that $M\left(\Delta_{n}\right)=M_{n}^{\prime}$.

We now assume $0 \leq i, k \leq n-1$. Then in $M_{n}$, we note that $a^{i} b^{i} a^{n} b^{k} a^{k} \sim a^{n}$; hence none of the elements of $N$ are zero and all of them are $\mathcal{J}$-equivalent. Now $a^{i+1} b^{i} a^{n} b^{k} \sim a^{n+1} b^{k} \sim 0$. But since $a^{i} b^{i} a^{n} b^{j} a^{j} \sim a^{n}$ which is not $0, i+1$ is the smallest power of $a$ for which this occurs. Similarly, $k+1$ is the smallest natural number $r$ with the property that $b^{i} a^{n} b^{k} a^{r} \sim 0$. Hence no two elements of the form $b^{i} a^{n} b^{k}$ with $0 \leq i, k \leq n-1$ are equal in $M_{n}$.

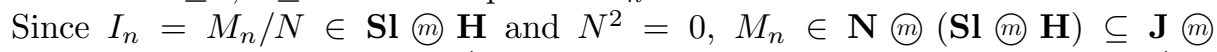
H. Now consider the pair $\left\{a^{n-1}, b\right\}$. These elements are regular; indeed, $b^{-1}=$ $a,\left(a^{n-1}\right)^{-1}=b^{n-1}$. So if $\mu: M_{n} \multimap G \in \mathbf{H}$ is a relational morphism and $a \mu g$, then $b \mu g^{-1}$. But $a^{n-1} \mu g^{n-1}=g^{-1}$ since $\mathbf{H}$ satisfies $x^{n}=1$, so $\left\{a^{n-1}, b\right\}$ is H-pointlike. Now, since $b^{n}=0$,

$$
a^{n-1}\left(a^{n-1}\right)^{-1} b b^{-1}=a^{n-1} b^{n-1} b a=0 .
$$

On the other hand,

$$
a^{n-1} b^{-1}=a^{n-1} a=a^{n} \neq 0 .
$$

Thus, by Theorem [7.22, $M_{n} \notin \mathbf{J} * \mathbf{H}$.

For example, $M_{2}=M_{2}^{\prime}$ is a 10 element monoid with the following $\mathcal{J}$-class structure.

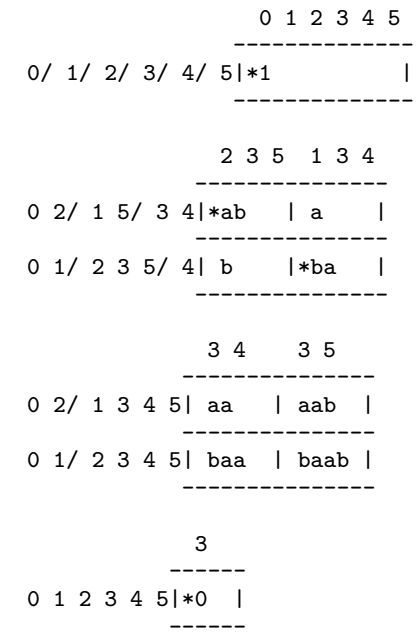

7.6. Other Applications to the Theory of Pseudovarieties. This subsection will be more of interest to specialists in finite semigroup theory; therefore, we have made less of an effort to be self-contained. The following definition is due to Ash [10]; see [3] for a more general concept which makes sense for pseudovarieties of monoids. Let $M$ be a monoid and $\Gamma$ a finite, oriented graph (it need not be connected). A labeling of $\Gamma$ over $M$ is an orientation-preserving graph morphism 
$\delta: \Gamma \rightarrow B_{M}$ (which is not required to be faithful). If $G$ is a group, a labeling $\delta$ of $\Gamma$ over $G$ is said to commute if, given any circuit $p$ in $\Gamma$, the product of the labels in $G$ is 1 , (recall that by the definition of a labeling, if $e$ is a positively oriented edge with label $g$, then $\bar{e}$ has $g^{-1}$ as its label). If $\mu: M \hookrightarrow N$ is a relational morphism and $\delta, \beta$ are labelings of a finite graph $\Gamma$ over $M$ and $N$, respectively, then we say that $\delta$ is $\mu$-related to $\beta$ if, for each edge $e \in E^{+}(\Gamma), e \delta \mu e \beta$. If $\mathbf{H}$ is a pseudovariety of groups, $M$ a finite monoid, $\Gamma$ a finite graph, and $\delta$ a labeling of $\Gamma$ over $M$, then $\delta$ is called $\mathbf{H}$-inevitable if, for any relational morphism $\mu: M \bullet G \in \mathbf{H}$, there is a labeling $\beta$ of $\Gamma$ over $G$ which commutes and which is $\mu$-related to $\delta$. For example, if $M$ is a finite monoid, a one edge, one vertex graph labeled by an element $m$ is $\mathbf{H}$-inevitable if and only if $m \in K_{\mathbf{H}}(M)$. More generally, a directed circuit of length $k$ labeled by $m_{1}, \ldots, m_{k}$ is $\mathbf{H}$-inevitable if and only if $\left(m_{1}, \ldots, m_{k}\right)$ is an $\mathbf{H}$-liftable $k$-tuple. A subset $X \subseteq M$ is $\mathbf{H}$-pointlike if and only if the natural labeling of the graph $\Gamma$ with two vertices, $v_{0}$ and $v_{1}$, and $|X|$ positively oriented edges, each with initial vertex $v_{0}$ and terminal vertex $v_{1}$, is $\mathbf{H}$-inevitable. Almeida defined $\mathbf{H}$ to be hyperdecidable [3] if there is an algorithm to determine whether a labeling of a finite graph by a finite monoid is $\mathbf{H}$-inevitable. This in turns implies that $\mathbf{H}$ has decidable membership problem, and that $K_{\mathbf{H}}, \mathbf{H}$-liftable $k$-tuples, and $\mathbf{H}$-pointlike sets are all computable. The following theorem is then proved by the author in [44].

Theorem 7.33. Let $M$ be a finite A-generated monoid, $\mathbf{H}$ an extension-closed pseudovariety of groups, and $\alpha_{\mathbf{H}}: M \rightarrow F G(A)$ the relational morphism considered in Section [5.2. Then a labeling $\delta$ of a finite graph $\Gamma$ over $M$ is $\mathbf{H}$-inevitable if and only there is a labeling $\beta$ of $\Gamma$ over $F G(A), \alpha_{\mathbf{H}}$-related to $\delta$, which commutes.

The following is also shown in [44.

Theorem 7.34. Let $\mathbf{H}$ be an extension-closed pseudovariety of groups for which there is an algorithm to compute membership in the closure of a finitely generated subgroup of a finite rank free group. Then $\mathbf{H}$ is hyperdecidable.

Now recall that if $M$ is a finite $A$-generated monoid, then $m \alpha_{\mathbf{H}}=c l_{\mathbf{H}}\left(L_{m}\right)$ where

$$
L_{m}=\left\{u \in A^{*} \mid[u]_{M}=m\right\} .
$$

In particular, if $M \in \mathbf{R} m \mathbf{H}$ or $\mathbf{L} m \mathbf{H}$, then, by Theorem 6.31

$$
m \alpha_{\mathbf{H}}=c l_{\mathbf{H}}\left(L_{m}\right)=c l_{\mathbf{G}}\left(L_{m}\right)=m \alpha_{\mathbf{G}} .
$$

Hence we get the following generalization of Theorem 7.21

Theorem 7.35. Let $\mathbf{H}$ be an extension-closed pseudovariety of groups, $M \in \mathbf{R} m \mathbf{H}$ or $\mathbf{L}(m) \mathbf{H}$, and $\delta$ a labeling of a finite graph $\Gamma$ over $M$. Then $\delta$ is $\mathbf{H}$-inevitable if and only if it is $\mathbf{G}$-inevitable. In particular, a subset of $M$ is $\mathbf{H}$-pointlike if and only if it is $\mathbf{G}$-pointlike.

We now obtain several remarkable consequences of this result. Recall that if $\mathbf{V}$ and $\mathbf{W}$ are pseudovarieties of monoids, then their join $\mathbf{V} \vee \mathbf{W}$ is the smallest pseudovariety containing them both. The following fundamental result on joins is due to the author 41,43 .

Theorem 7.36. Let $\mathbf{V}$ and $\mathbf{W}$ be pseudovarieties of monoids and $M$ a finite monoid. Then $M \in \mathbf{V} \vee \mathbf{W}$ if and only if there exists a relational morphism $\mu: M \multimap W \in \mathbf{W}$ such that no slice $\left\{\left(m_{1}, w\right),\left(m_{2}, w\right)\right\} \subseteq \mu$ is $\mathbf{V}$-pointlike. 
Corollary 7.37. Let $\mathbf{H}$ be an extension-closed pseudovariety of groups. Let $\mathbf{W} \subseteq$ $\mathbf{R}(\mathrm{H} \mathbf{H}$ be a pseudovariety of monoids. Then $\mathbf{H} \vee \mathbf{W}=(\mathbf{G} \vee \mathbf{W}) \cap(\mathbf{R} m \mathbf{H}) . A$ dual result holds replacing $\mathbf{R}$ with $\mathbf{L}$.

Proof. We just need to prove $M \in(\mathbf{G} \vee \mathbf{W}) \cap(\mathbf{R} m \mathbf{H})$ implies $M \in \mathbf{H} \vee \mathbf{W}$, the other direction being clear. But, by Theorem 7.36, $M \in(\mathbf{G} \vee \mathbf{W})$ implies that there is a relational morphism $\mu: M \multimap W \in \mathbf{W}$ such that no slice $\left\{\left(m_{1}, w\right),\left(m_{2}, w\right)\right\} \subseteq \mu$ is G-pointlike. But since $W \in \mathbf{W}$ and $M \in \mathbf{R} m \mathbf{H}, \mu \in \mathbf{R} m \mathbf{H}$ (viewing $\mu$ as a monoid). So, by Theorem 7.35, no such slice is $\mathbf{H}$-pointlike. Another application of Theorem [7.36] shows that $M \in \mathbf{H} \vee \mathbf{W}$.

So, for example, $\mathbf{R} \vee \mathbf{G}_{p}=(\mathbf{R} \vee \mathbf{G}) \cap(\mathbf{R}(\mathbf{H})$. Hence an algorithm to determine membership in $\mathbf{R} \vee \mathbf{G}$, would imply an only polynomially time slower algorithm for membership in $\mathbf{R} \vee \mathbf{G}_{p}$. The author has already shown [41, 43] that $\mathbf{J} \vee \mathbf{G}$ has decidable membership whence $\mathbf{J} \vee \mathbf{G}_{p}$ does as well by the above result (also proved by the author in [44]). We now obtain, by slightly more elementary methods, a joint result of Almeida and Weil 7]. Let ZE be the pseudovariety of monoids whose idempotents are central. Then $\mathbf{Z E}=(\mathbf{A} \cap \mathbf{C o m}) \vee \mathbf{G}[2]$ and $(\mathbf{A} \cap \mathbf{C o m}) \subseteq \mathbf{J}$. We use $\overline{\mathbf{H}}$ for the pseudovariety of all finite monoids whose subgroups lie in $\mathbf{H}$ and DLH for the pseudovariety of finite monoids whose regular $\mathbf{J}$-classes are left simple with subgroups in $\mathbf{H}$. First a lemma.

Lemma 7.38. Let $\mathbf{H}$ be any pseudovariety of groups. Then

$$
\mathbf{D L H} \subseteq \mathbf{R} \rightarrow \mathbf{H} .
$$

Proof. We show that if $M \in \mathbf{D L H}$ is $A$-generated, then, for every regular $\mathcal{R}$-class $X$ of $M, \operatorname{Sch}_{A}(X)$ is $\mathbf{H}$-extendible. It is well known that if $M \in \mathbf{D L H}$, then $X$ is a subgroup of $M$ in $\mathbf{H}$. Let $e$ be the identity of this subgroup and suppose $p, q \in X$ are such that $p \sim_{\mathbf{H}} q$. We use $\varphi_{\mathbf{H}}: F G(A) \rightarrow F G_{\mathbf{H}}(A)$ for the projection. Then there exists a sequence of words $\left\{w_{n}\right\}$ of $L_{p, q}$ such that $\left\{w_{n} \varphi_{\mathbf{H}}\right\}$ converges to 1 . Let $B \subseteq A$ be the set of letters appearing in some word of $\left\{w_{n}\right\}$. Then $\left\{w_{n} \varphi_{\mathbf{H}}\right\}$ converges to 1 in $F G_{\mathbf{H}}(B)$. Let $w_{n}^{\prime}$ be the element of $M$ obtained by replacing each letter $b$ occurring in $w_{n}$ by $e b$. Then, since $e$ is an identity for $X, p w_{n}^{\prime}=q$. We claim that, for each $b \in B, e b \in X$. First note that $e b \leq_{\mathcal{R}} e$. However, suppose $b$ occurs in $w_{j}$, then $p w_{j}^{\prime}=q$ implies that $q \leq \mathcal{J} e b$ whence $e b \mathcal{J} e$; we then deduce, since $M$ is a finite monoid, hence stable, that $e b \mathcal{R} e$. So we can define a morphism $\gamma: F G_{\mathbf{H}}(B) \rightarrow X$ by $b \mapsto e b$. It follows that, for $n$ large enough, $e=w_{n} \varphi_{\mathbf{H}} \gamma=w_{n}^{\prime}$. But $p w_{n}^{\prime}=q$ for all $n$, so since $p e=p$, we see that $p=q$.

Corollary 7.39. Let $\mathbf{H}$ be an extension-closed pseudovariety of groups. Then

$$
(\mathbf{A} \cap \mathbf{C o m}) \vee \mathbf{H}=\mathbf{Z E} \cap \overline{\mathbf{H}} .
$$

Proof. By Corollary 7.37,

$$
(\mathbf{A} \cap \mathbf{C o m}) \vee \mathbf{H}=((\mathbf{A} \cap \mathbf{C o m}) \vee \mathbf{G}) \cap(\mathbf{R}(m \mathbf{H})=\mathbf{Z E} \cap(\mathbf{R}(m \mathbf{H}) .
$$

Since $\mathbf{R}$ is aperiodic, $\mathbf{R} m \mathbf{H} \subseteq \overline{\mathbf{H}}$; hence we just need to show $\mathbf{Z E} \cap \overline{\mathbf{H}} \subseteq \mathbf{R} m \mathbf{H}$. But it is well known that if $M \in \mathbf{Z E}$, then the regular $\mathcal{J}$-classes of $M$ are groups, so

$$
\mathbf{Z E} \cap \overline{\mathbf{H}} \subseteq \mathbf{D L H} \subseteq \mathbf{R}[\mathbf{H} .
$$


The above result fails, for instance, if $\mathbf{H}=\mathbf{G}_{\text {com }}$ since any element of the left-hand side is commutative, while the monoid

$$
M=\left\langle x, y \mid x^{2}=y^{2}=x y x=y x y=0\right\rangle
$$

is in the right-hand side but not commutative. One can, in a similar manner, prove [7, Theorem 4.6] in the extension-closed case from the analogous result for G.

Our last goal is to prove some generalizations of the results of section 7.4. We shall merely sketch the proofs, as we wish neither to reproduce long proofs from other papers or introduce profinite monoids. Using that, for monoids in $\mathbf{R} m \mathbf{H}$ or $\mathbf{L}: \mathbf{H}$, the $\mathbf{H}$-inevitable and $\mathbf{G}$-inevitable graphs are the same, and using the argument of [5] Theorem 5.2], but with [4, Theorem 2.3] in place of [8, Theorem 5.3 , we see that the following result holds.

Theorem 7.40. Let $\mathbf{V}$ be a pseudovariety of monoids and $\mathbf{H}$ an extension-closed pseudovariety of groups. Then

$$
(\mathbf{V} * \mathbf{H}) \cap(\mathbf{R} * \mathbf{H})=(\mathbf{V} * \mathbf{G}) \cap(\mathbf{R} * \mathbf{H}) .
$$

In particular, if $\mathbf{V} \subseteq \mathbf{R}$, then

$$
\mathbf{V} * \mathbf{H}=(\mathbf{V} * \mathbf{G}) \cap(\mathbf{R} * \mathbf{H}) .
$$

A dual result holds for $\mathbf{L}$.

We note that Corollary 7.28 is just a special case of the above theorem. Also the above theorem implies that $(\mathbf{J} * \mathbf{H})=(\mathbf{J} * \mathbf{G}) \cap(\mathbf{R} * \mathbf{H})$. On the other hand, using that the analogous equality holds with $\mathbf{L}$ in place of $\mathbf{R}$, that $\mathbf{J} * \mathbf{G}=\mathbf{J} m \mathbf{G}$, and that $\mathbf{R}$ and $\mathbf{L}$ are local, it is straightforward to deduce that $\mathbf{J} * \mathbf{H}=\mathbf{J} m \mathbf{H}$ for extension-closed pseudovarieties of groups $\mathbf{H}$.

7.7. Conclusions and Conjectures. This paper has developed a geometric/combinatorial approach to problems in automata theory, particularly those closely tied to the theory of groups. The overriding theme has been to apply the techniques of geometric group theory to the strongly connected components of an automaton. In this manner, we have obtained a clearer, more efficient algorithm for computing the closure of a rational language in a free group with the profinite topology with the added bonus that if several languages are recognized by the same automaton, then one can compute all their closures simultaneously. In the process, we have seen that the computation of the regular elements of the $\mathbf{G}$-kernel requires only Marshall Hall's theorem and not the full strength of the Ribes and Zalesski1's Theorem. This perhaps explains why Rhodes and Tilson could prove the type II conjecture for regular elements almost 25 years before the general case was solved.

We have also clarified the results of Margolis, Sapir, and Weil [30] on inverse automata, incorporating their theory into a more general framework dealing with more complicated automata. This has led to a complete characterization of $\mathbf{R} m \mathbf{H}$ and its dual for pseudovarieties $\mathbf{H}$ of groups. This theory has also led to the first breakthrough in the problem $\mathbf{J}: \mathbf{H}=\mathbf{J} * \mathbf{H}$ since the theorem of Henckell and Rhodes at the start of the decade. However, while great progress has been made, we are still left with the question of classifying all pseudovarieties $\mathbf{H}$ for which the above equality holds.

The author conjectures, based on this work and [44], that $\mathbf{J}: \mathbf{H}=\mathbf{J} * \mathbf{H}$ holds if and only if $\left(\mathbf{G}_{c o m} \cap \mathbf{H}\right) * \mathbf{H}=\mathbf{H}$. Such pseudovarieties, if non-trivial, 
are called arborescent, and were studied in [7]. One result in that paper, which is essential for the Ribes and Zalesskiı result, is that the profinite Cayley graph for the free pro-H group generated by any finite set is an $\mathbf{H}$-tree. A result of 44 can be used to show that in this case, the fundamental group of an $\mathbf{H}$-extendible automaton is closed. We conjecture that the Ribes and Zalesskii result holds in this more general context. However, the proof of Ribes and Zalesskiu Theorem 37. 38. uses a property of extension-closed pseudovarieties not enjoyed by arborescent pseudovarieties in general. A preliminary step towards resolving the first conjecture would be to prove the following conjecture: suppose $\mathbf{H}$ is a non-trivial pseudovariety of groups satisfying a non-trivial group identity $(w=1)$; then equality fails. Our counterexamples were all based on this principle.

A final question to consider is whether closures of finitely generated subgroups of a finite rank free group are computable for $\mathbf{G}_{\text {sol }}$. This problem is probably more of a question for finite group theorists and is likely to be challenging.

\section{ADDENDUM}

Since the submission of this paper, there has been some further progress on some of the questions here investigated. In [47, the author has shown that $\mathbf{J} * \mathbf{H}=$ $\mathbf{J}(m) \mathbf{H}$ for arborescent pseudovarieties $\mathbf{H}$ by showing that the Ribes and Zalesski1 Theorem holds for products of two closed subgroups in this context. In [46], the author has completely characterized, for a pseudovariety of groups $\mathbf{H}$, those monoids recognizing pro-H open and closed recognizable languages, as well as studying the relationship between $\mathbf{J} * \mathbf{H}$ and various other pseudovariety operators.

\section{ACKNOWLEDGMENTS}

The author would like to thank: Stuart Margolis for many useful discussions over e-mail; Pedro Silva for assistance with monoid presentations and suggesting the construction of $\Delta$ in the proof of Theorem 6.21; Jorge Almeida for listening to various preliminary versions of these results; Bret Tilson for inspiring me to "do this the right way;" Misha Volkov for pointing out to me the results of [6]; John Stallings for letting me lecture on this work in his group theory seminar at Berkeley; and John Rhodes, both for teaching me semigroup theory, and for his continued interest in my work.

\section{REFERENCES}

1. D. Albert, R. Baldinger, J. Rhodes, Undecidability of the identity problem for finite semigroups, The Journal of Symb. Logic 57 (1992), 179-192. MR 93f:03030

2. J. Almeida, Finite Semigroups and Universal Algebra, World Scientific, 1994. MR 96b:20069

3. J. Almeida, Hyperdecidable pseudovarieties and the calculation of semidirect products, Internat. J. Algebra and Comput. 9 (1999), 241-261. MR 2001a:20102

4. J. Almeida and A. Escada, On the equation $\mathbf{V} * \mathbf{G}=\mathcal{E} \mathbf{V}$, Tech. Rep. CMUP 98-6, Univ. of Porto, 1998

5. J. Almeida and B. Steinberg, On the decidability of iterated semidirect products with applications to complexity, Proc. London Math. Soc. 80 (2000), 50-74. MR 2000j:20109

6. J. Almeida and M. V. Volkov, The gap between partial and full, Internat. J. Algebra and Comput. 8 (1998), 399-430. MR 99g:20102

7. J. Almeida and P. Weil, Relatively free profinite monoids: An introduction and examples, Semigroups, Formal Languages and Groups, J. B. Fountain, ed., vol. 466, Dordrecht, 1995, Kluwer Publ., 73-117. MR 2000f:20095

8. J. Almeida and P. Weil, Profinite categories and semidirect products, J. Pure and Appl. Algebra (1998) 123, 1-50. MR 99c:20080 
9. C. J. Ash, Finite semigroups with commuting idempotents, J. Austral. Math. Soc. Ser. A 43 (1987), 81-90. MR 88f:03045

10. C. J. Ash, Inevitable graphs: A proof of the type II conjecture and some related decision procedures, Internat. J. Algebra and Comput. 1 (1991), 127-146. MR 92k:20114

11. M. Benois, Parties rationnelles du groupe libre, C. R. Acad. Sci. Paris, Sér. A, 269 (1969), 1188-1190. MR 42:405

12. A. H. Clifford and G. B. Preston, The Algebraic Theory of Semigroups, Mathematical Surveys No. 7, AMS, Providence, RI, Vol. 1, 1961; Vol. 2, 1967. MR 24:A2627; MR 36:1558

13. M. Delgado, On the hyperdecidability of pseudovarieties of groups, Internat. J. Algebra and Comput. (to appear).

14. S. Eilenberg, Automata, Languages and Machines, Academic Press, New York, Vol A, 1974; Vol B, 1976. MR 58:26604a

15. D. B. A. Epstein with J. W. Cannon, D. F. Holt, S. V. F Levy, M. S. Paterson, W. P. Thurston, Word Processing in Groups, Jones and Bartlett, Boston-London, 1992. MR 93i:20036

16. R. Knast, Some theorems on graph congruences, RAIRO Inform. Théor. 17 (1983), 331-342. MR 86a:68055

17. K. Krohn and J. Rhodes, Algebraic theory of machines, I, Trans. Amer. Math. Soc. 116 (1965), 450-464. MR 32:5755

18. K. Krohn and J. Rhodes, Complexity of finite semigroups, Ann. of Math. 88 (1968), 128-160. MR 38:4591

19. R. H. Gilman, Groups with rational cross-sections, in "Essays in group theory", edited by S. M. Gersten and J. R. Stallings, P. U. P. Annals of Math series \#111, 1987, 175-183. MR 88g:20065

20. R. Gitik and E. Rips, On separability properties of groups, Internat. J. Algebra and Comput. 5 (1995), 703-717. MR 97e:20059

21. D. Gildenhuys and L. Ribes, Profinite groups and Boolean graphs, J. Pure Appl. Algebra 12 (1978), 21-47. MR 81g:20059

22. M. Hall, Jr. Coset representation in free groups, Trans. Amer. Math. Soc. 67 (1949), 421-432. MR 11:322e

23. M. Hall, Jr. A topology for free groups and related groups, Annals of Mathematics 52 (1950), 127-139. MR 12:158b

24. K. Henckell, S. Margolis, J.-E. Pin, and J. Rhodes, Ash's type II theorem, profinite topology and Malcev products. Part I, Internat. J. Algebra and Comput. 1 (1991), 411-436. MR 93h:20064

25. S. MacLane, Categories for the Working Mathematician, Springer-Verlag, New York, 1971. MR 50:7275

26. S. W. Margolis, private communication.

27. S. W. Margolis and J.-E. Pin, Inverse semigroups and varieties of finite semigroups, J. Algebra 110 (1987), 306-323. MR 89j:20066b

28. S. W. Margolis and J. C. Meakin, Free inverse monoids and graph immersions, Internat. J. Algebra and Comput. 3 (1993), 79-99. MR 94c:20105

29. S. W. Margolis, J. C. Meakin, and M. Sapir, Algorithmic problems, Semigroups, Formal Languages and Groups, J. B. Fountain, ed., vol. 466, Dordrecht, 1995, Kluwer Publ. MR 99e:20043

30. S. W. Margolis, M. Sapir, and P. Weil, Closed subgroups in pro-V topologies and the extension problem for inverse automata, Internat. J. Algebra and Comput. (to appear).

31. O. Matz, A. Miller, A. Pothoff, W. Thomas, and E. Valkema, Report on the program AMoRE, Tech. Rep. 9507, Christian Albrechts Universit at, Kiel, 1994.

32. W. D. Munn, Free inverse semigroups, Proc. London Math. Soc. 30 (1974), 385-404. MR 48:447

33. G. Niblo, Separability properties of free groups and surface groups, J. Pure Appl. Algebra $\mathbf{7 8}$ (1992), 77-84. MR 92m:20019

34. J.-E. Pin and C. Reutenauer, A conjecture on the Hall topology for the free group, Bull. London Mat. Soc. 23 (1991), 356-362. MR 92g:20035

35. J. Rhodes, Undecidability, automata and pseudovarieties of finite semigroups, Internat. J. Algebra and Comput. 9 (1999), 455-473. MR 2000j:20012

36. J. Rhodes and B. Steinberg, Pointlike sets, hyperdecidability, and the identity problem for finite semigroups, Internat. J. Algebra and Comput. 9 (1999), 475-481. MR 2000k:20075 
37. L. Ribes and P. A. Zalesskiĭ, On the profinite topology on a free group, Bull. London Math. Soc. 25 (1993), 37-43. MR 93j:20062

38. L. Ribes and P. A. Zalesski1, The pro-p topology of a free group and algorithmic problems in semigroups, Internat. J. Algebra and Comput. 4 (1994), 359-374. MR 96e:20046

39. J.-P. Serre, Trees, Springer-Verlag, Heidelberg, 1980. MR 82c:20083

40. J. Stallings, Topology of finite graphs, Inv. Math. 71 (1983), 551-565. MR 85m:05037a

41. B. Steinberg, On pointlike sets and joins of pseudovarieties, Internat. J. Algebra and Comput. 8 (1998), 203-231. MR 99g:20104

42. B. Steinberg, Monoid kernels and profinite topologies on the free Abelian group, Bull. of the Austral. Math. Soc. 60 (1999), 391-402. MR 2000h:20120

43. B. Steinberg, On algorithmic problems for joins of pseudovarieties, Semigroup Forum 62 (2001), 1-40.

44. B. Steinberg, Inevitable Graphs and Profinite Topologies: Some solutions to algorithmic problems in monoid and automata theory stemming from group theory, Internat. J. Algebra and Comput. 11 (2001), 25-71.

45. B. Steinberg, A note on the equation $\mathbf{P H}=\mathbf{J} * \mathbf{H}$, Semigroup Forum (to appear)

46. B. Steinberg, Polynomial closure and topology, Internat. J. Algebra and Comput. 10 (2000), 603-624. CMP 2001:01

47. B. Steinberg, Inverse automata and profinite topologies on a free group, J. Pure and Appl. Algebra (to appear).

48. B. Tilson, Type II redux, Semigroups and Their Applications, ed. S. M. Goberstein and P. M. Higgins, Reidel, Dordecht, 1987, 201-205. MR 88k:20086

49. B. Tilson, Categories as algebra: An essential ingredient in the theory of monoids, J. Pure and Appl. Algebra 48 (1987), 83-198. MR 90e:20061

50. P. Weil, Some results on the dot-depth hierarchy, Semigroup Forum 46 (1993), 352-370. MR 93m:20089

51. E. Zel'manov, More on Burnside's Problem, Combinatorial and geometric group theory. Proceedings of a workshop held at Heriot-Watt University, Edinborough GB Spring of 93, ed. A. Duncan et al., London Math. Soc. Lec. Note Ser. 204, Cambridge, 1995, 314-321. MR 96k:20080

Faculdade de Ciências, da Universidade do Porto, 4099-002 Porto, Portugal

E-mail address: bsteinbg@agc0.fc.up.pt 